\title{
Exploiting Incoherent Sampling to Provide Electronic Protection Against Digital Radio Frequency Memory Jammers
}

\author{
by
}

Nick D. Deloyer, B.Eng.

\author{
A Thesis submitted to the \\ Faculty of Graduate and Postdoctoral Affairs \\ in partial fulfilment of \\ the requirements for the degree of \\ Master of Applied Science \\ in
}

Electrical and Computer Engineering

Ottawa-Carleton Institute for Electrical \& Computer Engineering

Department of Electronics

Faculty of Engineering

Carleton University

Ottawa, Ontario, Canada

April 2012

Copyright (C) Nick Deloyer 2012 
Library and Archives

Canada

Published Heritage

Branch

395 Wellington Street

Ottawa ON K1A ON4

Canada
Bibliothèque et

Archives Canada

Direction du

Patrimoine de l'édition

395 , rue Wellington

Ottawa ON K1A ON4

Canada
Your file Votre référence

ISBN: 978-0-494-91579-0

Our file Notre référence

ISBN: $978-0-494-91579-0$
NOTICE:

The author has granted a nonexclusive license allowing Library and Archives Canada to reproduce, publish, archive, preserve, conserve, communicate to the public by telecommunication or on the Internet, loan, distrbute and sell theses worldwide, for commercial or noncommercial purposes, in microform, paper, electronic and/or any other formats.

The author retains copyright ownership and moral rights in this thesis. Neither the thesis nor substantial extracts from it may be printed or otherwise reproduced without the author's permission.
AVIS:

L'auteur a accordé une licence non exclusive permettant à la Bibliothèque et Archives Canada de reproduire, publier, archiver, sauvegarder, conserver, transmettre au public par télécommunication ou par l'Internet, prêter, distribuer et vendre des thèses partout dans le monde, à des fins commerciales ou autres, sur support microforme, papier, électronique et/ou autres formats.

L'auteur conserve la propriété du droit d'auteur et des droits moraux qui protege cette thèse. $\mathrm{Ni}$ la thèse ni des extraits substantiels de celle-ci ne doivent être imprimés ou autrement reproduits sans son autorisation.
In compliance with the Canadian Privacy Act some supporting forms may have been removed from this thesis.

While these forms may be included in the document page count, their removal does not represent any loss of content from the thesis.
Conformément à la loi canadienne sur la protection de la vie privée, quelques formulaires secondaires ont été enlevés de cette thèse.

Bien que ces formulaires aient inclus dans la pagination, il n'y aura aucun contenu manquant. 


\section{Abstract}

Digital Radio Frequency Memory (DRFM) jammers are used by forces in Electronic Attack (EA) to neutralize weapons and hamper their ability to maintain accurate situational awareness. A currently unexploited trait of a DRFM, in terms of Electronic Protection (EP), is that it has an added digitization process in the DRFM signal path compared to a skin return. In addition, this digitization is done with incoherent sampling. An EP technique, called the Concatenated Random Noise (CRN) Technique, is proposed to take advantage of this DRFM trait to discriminate against jamming. It concatenates a short random noise pulse, with frequency components very close to the Nyquist rate, to the radar pulse. Due to incoherent sampling, the DRFM slightly distorts the random noise pulse when digitized. An EP processor on the radar, using a matched filter, is able to detect this slight distortion, and therefore discriminate between DRFM and skin returns. A simulation of the CRN technique and performance metrics are also presented. 


\section{Acknowledgments}

The support of my family, particularly my wife Shellie, was instrumental in completing the required courses and the work for this document. Her encouragement pushed me to study and continue developing my ideas, even when $I$ was uncertain, and her understanding was greatly appreciated when this work, combined with my full-time job, took a great amount of time away from us. I could not have done it without her!

The guidance and tutelage from my thesis advisor, Dr. Jim Wight, cannot be understated. He gently steered my ideas and inspired me to pursue them. I always left our aperiodic update meetings with a renewed sense of accomplishment in my work. He also took time out of his busy schedule to facilitate a one-on-one directed study in Radar Systems. I also need to thank Christina O'Regan for getting me established at Defence Research and Development Canada (DRDC) in Ottawa to work on this thesis. The access she provided to the DRDC library, IT systems, MATLAB licences and knowledge was invaluable. Many thanks to Dr. Jeff Lange and Dr. Sreerman Rajan for their interest and guidance.

Finally, I need to thank the Canadian Forces for it's support in the completion of my Masters, providing both financial compensation for expenses incurred and educational leave from my full-time position to attend classes and to draft this document.

Nick Deloyer

Ottawa, Ontario

April 2012 


\section{Table of Contents}

$\begin{array}{ll}\text { Abstract } & \text { iii }\end{array}$

Acknowledgments iv

Table of Contents $\quad$ v

List of Tables $\quad$ ix

List of Figures $\quad$ x

List of Acronyms xii

List of Symbols $\quad$ Xv

1 Introduction 1

1.1 The EA-EP Duel . . . . . . . . . . . . . . . . . 2

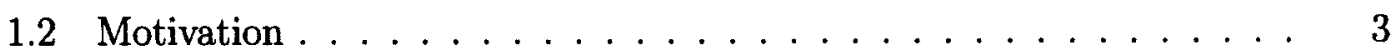

1.3 Problem Statement . . . . . . . . . . . . . . . . 4

1.4 Contributions ........................ 4

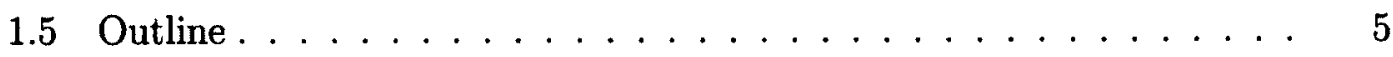

2 Review of Relevant Theory $\quad 6$

2.1 Digital Radio Frequency Memory Jammers . . . . . . . . . . . . . 6

2.1.1 Architecture ................. 6 
2.1 .2 Jamming Techniques $\ldots \ldots \ldots \ldots \ldots \ldots$

2.2 Digital Signal Processing (DSP) $\ldots \ldots \ldots \ldots \ldots \ldots$

2.2 .1 Sampling Theorem . . . . . . . . . . . . . . 10

2.2.2 Digital Finite Impulse Response (FIR) Filters . . . . . . . 12

2.3 Matched Filtering . . . . . . . . . . . . . . . 13

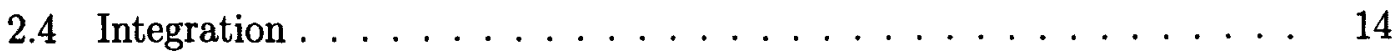

2.4.1 Non-coherent Integration $\ldots \ldots \ldots \ldots \ldots$

2.4 .2 Binary Integration $\ldots \ldots \ldots \ldots \ldots \ldots$

2.5 Random Variables . . . . . . . . . . . . . . . . . . . . 15

3 Current EP Techniques Countering DRFM Jammers 17

3.1 Separation and Filtering $\ldots \ldots \ldots \ldots \ldots \ldots$

3.1.1 Stretch Processing $\ldots \ldots \ldots \ldots$

3.1 .2 Rapid Relock . . . . . . . . . . . . . . . . 20

3.1.3 Narrow Gate Monitoring . . . . . . . . . . . . . . . 21

3.1.4 Frequency Diversity . . . . . . . . . . . . . . . 24

3.2 Denial . . . . . . . . . . . . . . . . . . . 24

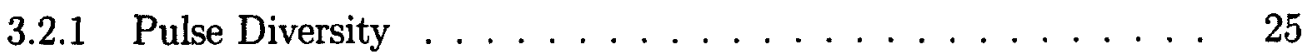

3.2.2 Ultrawideband Bandlimited Random Noise Waveforms . . . . 26

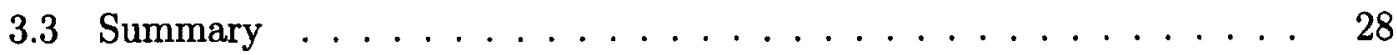

4 Radar Electronic Protection using Concatenated Random Noise (CRN) 30

4.1 Concept . . . . . . . . . . . . . . . . . 32

4.1 .1 Sampling Delay . . . . . . . . . . . . . . . . 32

4.1.2 PDF of Sampling Delay $\left(f_{\tau}(z)\right) \ldots \ldots \ldots \ldots \ldots$

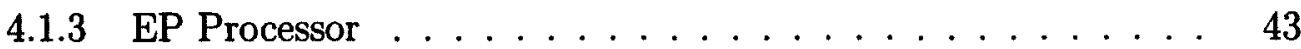

4.2 The CRN Technique, Design and Simulation . . . . . . . . . 44 


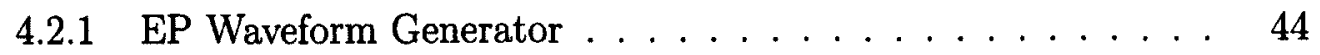

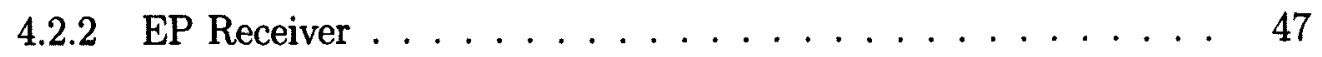

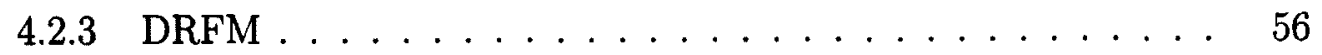

4.2 .4 Simulation Results . . . . . . . . . . . . . . 58

4.3 Summary $\ldots \ldots \ldots \ldots \ldots \ldots \ldots \ldots \ldots \ldots$

5 Results $\quad 64$

5.1 Standard CRN Pulse $\ldots \ldots \ldots \ldots \ldots \ldots \ldots$

5.1 .1 Baseline . . . . . . . . . . . . . . . 65

$5.1 .2 \quad \Delta$ SNR Varied $\ldots \ldots \ldots \ldots \ldots \ldots \ldots \ldots$

5.1 .3 Center Frequency $\left(\omega_{c}\right)$ Varied $\ldots \ldots \ldots \ldots$

5.1 .4 Bandwidth $(B)$ Varied . . . . . . . . . . . . 72

5.2 Short CRN Pulse $\ldots \ldots \ldots \ldots \ldots \ldots \ldots$

5.3 Long CRN Pulse $\ldots \ldots \ldots \ldots \ldots \ldots \ldots$

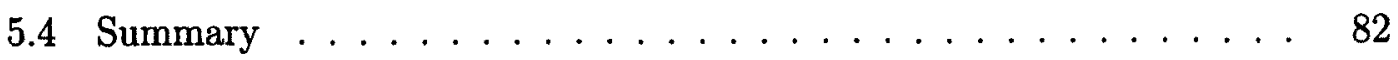

6 Conclusion $\quad 86$

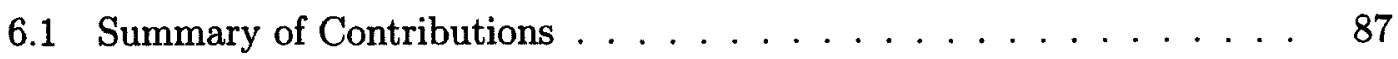

6.2 Future Research . . . . . . . . . . . . . . . . . . 88

6.2 .1 Adaptive Threshold . . . . . . . . . . . . . . . 88

6.2 .2 False Targets $\ldots \ldots \ldots \ldots \ldots \ldots$

6.2 .3 Quantization Noise and Jitter . . . . . . . . . . . . 90

6.2.4 Doppler Shift . . . . . . . . . . . . . . . . . . . 90 90

6.2.5 Lower Bandwidth Requirements . . . . . . . . . . . . . 92

6.2.6 Demonstrate for Different Radar Types . . . . . . . . . . 92

6.2.7 Optimization of Integration Scheme . . . . . . . . . . . 93

6.2.8 Simulation in Hardware with an Analog Signal Path . . . . . 94

6.2.9 Determine $f_{\tau}(z)$ for Land, Naval and Space Engagements . . . 94 
6.2.10 Integration with Existing EP Techniques . . . . . . . . . 94

6.2 .11 Advanced Tracking . . . . . . . . . . . . . . . 95

6.2 .12 Radar Resource Allocation . . . . . . . . . . . . . . 95

$\begin{array}{ll}\text { List of References } & 97\end{array}$

Appendix A Random Delay for HPRF Radars $\quad 99$ 


\section{List of Tables}

3.1 Summary of Current EP Techniques countering DRFMs _ . . . . . 29

4.1 Typical Fighter Aircraft Specification Values . . . . . . . . . . 36

4.2 Parameter Descriptions for the CRN Technique Simulation . . . . . 59

4.3 Performance Metrics for the CRN Technique Simulation . . . . . . 60

5.1 Simulation Parameters for Standard CRN Pulse Baseline . . . . . . 65

5.2 Summary of Results for $\triangle S N R$ Varied $\ldots \ldots \ldots$. . . . . . 68

5.3 Summary of Results for Standard CRN Pulse, B Varied . . . . . . . . 74

5.4 Summary of Results for Short CRN Pulse, B Varied . . . . . . . . . 77

5.5 Summary of Results for Long CRN Pulse, $B$ Varied . . . . . . . . . . 79

5.6 Summary of CRN Technique Simulation Results . . . . . . . . . 83 


\section{List of Figures}

2.1 Block Diagram of Generic DRFM Jammer . . . . . . . . . . . 7

2.2 Random Variable Mapping . . . . . . . . . . . . . . . . . . . . 16

3.1 Stretch Waveform and Processing . . . . . . . . . . . . . 19

3.2 Simple Leading Edge Tracking . . . . . . . . . . . . . . . . . . . 21

3.3 Example of Narrow Gate Monitoring . . . . . . . . . . . . . . 23

3.4 Jammer Model Performance . . . . . . . . . . . . . . . 27

4.1 Example of Incoherent Sampling of Gaussian Noise . . . . . . . . . 33

4.2 Development of $f_{V_{q}}(y) \ldots \ldots \ldots \ldots$

4.3 Development of $f_{R_{q}}(x) \ldots \ldots \ldots \ldots$

4.4 Development of $f_{\tau_{q, 1}}(z)$ (Unconstrained) $\ldots \ldots . \ldots . \ldots 40$

4.5 Development of $f_{\tau_{q, 1}}(z)$ (Constrained to one sample period) . . . . . 41

4.6 Development of $f_{\tau_{q, 5}}(z)$ (Constrained to one sample period) . . . . 42

4.7 Development of $f_{\tau_{q, 9}}(z)$ (Constrained to one sample period) . . . . . 43

4.8 Flowchart for the EP Waveform Generator . . . . . . . . . . . . 45

4.9 Flowchart for the EP Receiver . . . . . . . . . . . . . . . . 48

4.10 Typical Matched Filter Result . . . . . . . . . . . . . . 52

4.11 Flowchart for the DRFM Jammer . . . . . . . . . . . . . 56

4.12 Flowchart of the CRN Technique Simulation . . . . . . . . . 58

4.13 Example of a High Granularity Simulation Results Plot . . . . . . . 61

4.14 Example of a Low Granularity Simulation Results Plot . . . . . . . . 62 
5.1 Standard CRN Pulse, Low Granularity, Baseline Threshold Calculation 66

5.2 Standard CRN Pulse, High Granularity, Baseline Threshold Calculation $\left(S N R_{\text {radar }_{\text {skin }}}=-1 d B\right) \ldots \ldots \ldots 7$

5.3 Standard CRN Pulse, Low Granularity, Effect of Varying $\Delta S N R$. . 69

5.4 Standard CRN Pulse, High Granularity, Effect of Varying $\Delta S N R$. $\quad 70$

5.5 Standard CRN Pulse, Low Granularity, Effect of Varying $\omega_{c}$. . . . . 71

5.6 Standard CRN Pulse, Low Granularity, Effect of Varying $B$. . . . . 73

5.7 Standard CRN Pulse, High Granularity, Effect of Varying $B$ at Minimum Achievable SNR . . . . . . . . . . . . . . . . 75

5.8 Short CRN Pulse, Low Granularity, Effect of Varying B . . . . . . . . 76

5.9 Short CRN Pulse, High Granularity, Effect of Varying $B$ at Minimum Achievable SNR . . . . . . . . . . . . . 78

5.10 Long CRN Pulse, Low Granularity, Effect of Varying $B$. . . . . . . 80

5.11 Long CRN Pulse, High Granularity, Effect of Varying $B$ at Minimum Achievable SNR . . . . . . . . . . . . . . . 81

5.12 Relationship between Bandwidth-Time Product $\left(B T_{P D_{c r n}}\right)$ and Minimum Achievable $S N R_{\text {radar }_{\text {skin }}} \ldots \ldots \ldots \ldots$. . . . . . . 84

A.1 Development of $f_{V_{q}}(y)$, HPRF Case . . . . . . . . . 100

A.2 Development of $f_{R_{q}}(x)$, HPRF Case . . . . . . . . . 101

A.3 Development of $f_{\tau_{q, 1}}(z)$, HPRF Case (Unconstrained) . . . . . . . 102

A.4 Development of $f_{\tau_{q, 1}}(z)$, HPRF Case (Constrained to one sample period) 103

A.5 Development of $f_{\tau_{q}, 5}(z)$, HPRF Case (Constrained to one sample period) 103

A.6 Development of $f_{\tau_{q, 9}}(z)$, HPRF Case (Constrained to one sample period) 104 


\section{List of Acronyms}

\begin{tabular}{ll}
\hline Acronyms & Definition \\
\hline ADC & Analog to Digital Converter \\
AGC & Automatic Gain Control \\
BLRN & Bandlimited Random Noise \\
BPF & Band Pass Filter \\
CFAR & Constant False Alarm Rate \\
CPI & Coherent Processing Interval \\
CRN & Concatenated Random Noise \\
DAC & Digital to Analog Converter \\
DRFM & Digital Radio Frequency Memory \\
DSP & Digital Signal Processing; or \\
& Digital Signal Processor \\
& Electronic Attack \\
&
\end{tabular}




\begin{tabular}{|c|c|}
\hline ECM & Electronic Counter Measure \\
\hline EM & Electromagnetic \\
\hline EMS & Electromagnetic Spectrum \\
\hline EP & Electronic Protection \\
\hline ES & Electronic Support \\
\hline EW & Electronic Warfare \\
\hline FIR & Finite Impulse Response \\
\hline FPGA & Field Programmable Gate Array \\
\hline GML & Generalized Maximum Likelihood \\
\hline HPRF & High Pulse Repetition Frequency \\
\hline IIR & Infinite Impulse Response \\
\hline ISAR & Inverse Synthetic Aperture Radar \\
\hline LFM & Linear Frequency Modulation \\
\hline LO & Local Oscillator \\
\hline LPF & Low Pass Filter \\
\hline MPRF & Medium Pulse Repetition Frequency \\
\hline PD & Pulse Duration \\
\hline PDF & Probability Density Function \\
\hline PRF & Pulse Repetition Frequency \\
\hline
\end{tabular}




\begin{tabular}{ll} 
PRI & Pulse Repetition Interval \\
RCS & Radar Cross Section \\
RF & Radio Frequency \\
RGPO & Range Gate Pull Off \\
SAR & Synthetic Aperture Radar \\
SNR & Signal-to-Noise Ratio \\
UWB & Ultrawideband \\
VGPO & Velocity Gate Pull Off \\
\hline
\end{tabular}




\section{List of Symbols}

\begin{tabular}{ll}
\hline Symbols & Definition \\
\hline$B$ & bandwidth \\
$b_{l}$ & CRN pulse matched filter coefficients \\
$D$ & decision from the m of n detector \\
$D_{d r f m}(k)$ & $k^{\text {th }}$ decision from DRFM signal path of simulation \\
$D_{s k i n}(k)$ & $k^{\text {th }}$ decision from skin signal path of simulation \\
$D_{T}$ & decision from the threshold detector \\
$f_{s}$ & sampling frequency \\
$f_{s_{d}}$ & Pampling frequency of the DRFM \\
$f_{s_{r}}$ & PDF of engagement velocity \\
$f_{R_{q}}(x)$ & PDF of sampling delay due to range for CPI q \\
$f_{v}(y)$ &
\end{tabular}




\begin{tabular}{|c|c|}
\hline$f_{\Delta_{P R I}}(y)$ & PDF of range change during one PRI \\
\hline$f_{\tau}(z)$ & PDF of sampling delay $\tau$ \\
\hline$g$ & filter guard value \\
\hline$H_{0}$ & hypothesis that $\left|y^{2}\right|$ indicates a skin return \\
\hline$H_{1}$ & hypothesis that $\left|y^{2}\right|$ indicates a DRFM return \\
\hline$i_{p}$ & $\begin{array}{l}\text { the index of the maximum value of } y(n) \text { found by the mag- } \\
\text { nitude detector for pulse } p\end{array}$ \\
\hline$j$ & number of simulations to run \\
\hline$k$ & number of trials in a simulation \\
\hline$l$ & index for the CRN pulse matched filter $b_{l}$ \\
\hline$n$ & number of binary inputs to the $m$ of $n$ detector \\
\hline$N_{c r n}$ & length of $x_{c r n}$ \\
\hline$m$ & threshold for the $m$ of $n$ detector \\
\hline$p$ & pulse number \\
\hline$P_{D_{s k i n}}$ & skin probability of detection \\
\hline$P_{F A_{d r f m}}$ & DRFM false alarm probability \\
\hline$P_{D_{d r f m}}$ & DRFM probability of detection \\
\hline$P_{N D_{d r f m}}$ & DRFM probability of no detection \\
\hline$q$ & CPI number \\
\hline
\end{tabular}




\begin{tabular}{|c|c|}
\hline$R_{q}$ & sampling delay due to range for CPI $q$ \\
\hline$S N R_{d r f m}$ & SNR at the DRFM receiver \\
\hline$S N R_{\text {radar }_{\mathrm{d} r f m}}$ & SNR of the DRFM return at the radar receiver \\
\hline$S N R_{\text {radar }_{\text {skin }}}$ & SNR of the skin return at the radar receiver \\
\hline$t_{D}$ & time to detection decision \\
\hline$T$ & threshold of threshold detector \\
\hline$T_{C P I}$ & period of a CPI \\
\hline$T_{P R I}$ & period between pulses, pulse repetition interval \\
\hline$T_{s}$ & sampling period \\
\hline$v$ & velocity \\
\hline$V_{q}$ & sampling delay due to velocity for CPI $q$ \\
\hline$x_{c r n}(n)$ & original CRN signal \\
\hline$x_{c r n+\tau}(n)$ & $\begin{array}{l}\text { CRN signal with } x_{\tau} \text { distortion and } x_{n} \text { noise received by } \\
\text { radar after ADC }\end{array}$ \\
\hline$x_{n}(n)$ & white gaussian noise signal \\
\hline$x_{p}(n)$ & $\begin{array}{l}\text { abstract signal representing } x_{c r n+\tau} \text { after radar receiver fil- } \\
\text { tering, ready for EP Processing }\end{array}$ \\
\hline$x_{p_{d r f m}}(n)$ & DRFM return CRN signal ready for EP processing \\
\hline$x_{p_{s k i n}}(n)$ & skin return CRN signal ready for EP processing \\
\hline
\end{tabular}




\begin{tabular}{|c|c|}
\hline$x_{\tau}(n)$ & sampling delay distortion signal \\
\hline$x_{\tau_{d}}(n)$ & sampling delay distortion caused by DRFM receiver \\
\hline$x_{\tau_{r}}(n)$ & sampling delay distortion caused by radar receiver \\
\hline$|y|^{2}$ & output of square law detector \\
\hline$\left|y_{d r f m}\right|^{2}$ & output of the square law detector, given a DRFM input \\
\hline$\left|y_{s k i n}\right|^{2}$ & output of the square law detector, given a skin input \\
\hline$y(n)$ & abstract output signal of matched filter \\
\hline$y_{c r n}(n)$ & matched filter result for $x_{c r n}(n)$ \\
\hline$y_{n}(n)$ & matched filter result for $x_{n}(n)$ \\
\hline$y_{d r f m}(n)$ & matched filter result for DRFM return, $x_{p_{d r f m}}$ \\
\hline$y_{s k i n}(n)$ & matched filter result for skin return, $x_{p_{s k i n}}$ \\
\hline$y_{\tau_{d}}(n)$ & matched filter result for $x_{\tau_{d}}$ \\
\hline$y_{\tau_{r}}(n)$ & matched filter result for $x_{\tau_{r}}$ \\
\hline$y_{\max }$ & maximum value of $y(n)$, output of the magnitude detector \\
\hline$y_{m a x_{d r f m}}$ & output of the magnitude detector, given a DRFM input \\
\hline$y_{\text {max }}$ skin & output of the magnitude detector, given a skin input \\
\hline$\Delta R_{C P I}$ & range change during one $\mathrm{CPI}$ \\
\hline$\Delta R_{P R I}$ & range change during one $\mathrm{PRI}$ \\
\hline$\Delta S N R$ & difference in SNR between $S N R_{\text {radar }_{d r f m}}$ and $S N R_{\text {radar }_{\text {skin }}}$ \\
\hline
\end{tabular}


$\tau$

$\tau_{d}$

$\tau_{q, p}$

$\tau_{r}$

$\omega_{c}$ sampling delay (random variable)

sampling delay at DRFM receiver

sampling delay for pulse $p$ and CPI $q$ (used in derivation

of $f_{\tau}(z)$ only)

sampling delay at radar receiver

center frequency 


\section{Chapter 1}

\section{Introduction}

The roots of Electronic Warfare (EW) can be traced back to the World Wars, before the term EW had been coined. During the Second World War, in The Battle of the Beams, one of the first uses of deception in EW was executed. The British countered a German navigational aid, which guided bombers over London with directional radio frequency $(\mathrm{RF})$ beams, by receiving the German signals and retransmitting them in different directions to confuse German bombers [1]. Since this simple but clever use of electronics were employed, EW has become increasingly important to guarantee combat effectiveness.

EW is defined as a military action whose objective is to control the Electromagnetic (EM) Epectrum (EMS). To accomplish this objective, both offensive Electronic Attack (EA) and defensive Electronic Protection (EP) actions are required. In addition, Electronic Support (ES) actions are necessary to supply the intelligence and threat recognition that allow implementation of both EA and EP [2]. Control of the EMS allows friendly forces to freely use the EMS to locate, identify and engage the enemy with radars and deny the same capability to the enemy forces. Although EW is used in other operational contexts, such as communications and navigation, its application to radar is the traditional mission of EW and the focus of this thesis. 


\subsection{The EA-EP Duel}

In order to employ EW on the battlefield, a great amount of science and engineering is required to field and configure the equipment used in EW. This has started its own war of sorts between the scientists and engineers of adversaries. To introduce this war or duel, the definitions of EA and EP are given followed by an brief example.

EA involves the use of EM energy, directed energy, or anti-radiation weapons to attack personnel, facilities, or equipment with the intent of degrading, neutralizing or destroying enemy combat capability [3]. EA can be both active and passive. Examples of active EA include jammers and anti-radiation missiles; examples of passive EA include chaff and decoys.

EP involves actions taken to protect personnel, facilities, and equipment from any effects of friendly or enemy use of the electromagnetic spectrum that degrade, neutralize, or destroy friendly combat capability [3]. Examples of EP include emission control, electronic hardening, 'low observability' or stealth, and many more. However the most important form of EP is the measures taken to embed various techniques into electronic equipment to make them less vulnerable to EA [2].

EA and EP techniques are typically closely related to one another. For example, an adversary may deploy a simple noise jammer against one of our radars-a form of EA. Once our forces notice the employment of the noise jammer, we will develop and employ a countermeasure, such as embedding a frequency diversity technique into our radar-a form of EP. Once the adversary has recognized that their jammer's effectiveness has fallen, they will likely employ ES to determine the EP technique our forces have employed. Using the gathered intelligence they now have the opportunity to employ a counter-countermeasure by upgrading the jammer. This $E A-E P$ duel between adversaries can go through many iterations and has been since the World Wars. In older sources, one may encounter the terms Electronic Countermeasures 
(ECM) and Electronic Counter-countermeasures (ECCM). However, to avoid confusion from using the word 'counter' in both terms, the new terminology used is EA and EP [3].

\subsection{Motivation}

It is a necessity to remain a measure ahead of adversaries in this EA-EP duel on the modern battlefield. The current cutting edge in EA is the Digital Radio Frequency Memory (DRFM) jammer. According to Barry Manz they have become an "indispensable component" of the EA-EP duel and they are "capable of performing truly impressive feats of magic on their captured signals" [4]. A DRFM jammer is able to record a radar pulse, perform signal processing and retransmit the pulse fast enough and with high enough fidelity that current radar receivers are not able to distinguish between the skin return and the jammer return. Using specific processing techniques, the DRFM is able to propose a scenario in which its host platform (the radar's target) has a different radar cross-section, range, speed, or location. It can also create false targets to confuse our radar and more [4]. Without an effective EP technique, the radar will be confused by mislocated and/or misidentified targets, confusing our forces and rendering our forces' radar guided weapons virtually useless.

Some EP techniques to counter the modern DRFM jammer have been proposed and a few are in use. To date there are few successful simulations of proposed EP techniques, in open literature, that detect and identify the jamming signal via contents of the returned signal itself. Most current techniques use simple temporal, spatial, doppler or average power information of the pulse, but this could be ambiguous with real targets. 


\subsection{Problem Statement}

The aim of this thesis is to develop an EP technique that allows a radar to differentiate between a DRFM jammer return and the skin return of the DRFM's host platform. Furthermore, some goals of the EP technique are that it will not alter the effectiveness of the radar in its primary role, and it will use a vulnerability of the DRFM that the designer cannot easily counter. It will be assumed that the radar receiver captures many return pulses, some skin returns and some DRFM returns. Further, it will be assumed that the radar receiver down-converts, samples and isolates the pulses before passing them to the EP processor. The output of this processor will indicate to the radar receiver whether the pulses were a skin return or a DRFM jammer signal.

\subsection{Contributions}

The result of the research for this thesis was a novel EP technique-the CRN technique- which is able to discriminate between DRFM and skin returns, within all the constraints mentioned above, as well as a MATLAB simulation to analyse its effectiveness and begin to examine factors affecting its performance. The CRN technique is described in detail in Chapter 4. In brief, the CRN technique concatenates a random noise pulse to the regular radar pulse. The radar transmits and receives this pulse pair. Once received, the radar decatenates the CRN pulse and passes it to the $\mathrm{EP}$ receiver for detection processing. The EP receiver uses a matched filter as its first step in the detection process. The result of the matched filter has, on average, a higher magnitude from a skin return than from a DRFM return. The small difference in magnitude is grown through integration and a threshold applied to determine if the pulse being processed is a skin return or DRFM return. 


\subsection{Outline}

General background concepts and radar signal processing techniques necessary for the comprehension of this thesis will be reviewed in Chapter 2. Chapter 3 briefly discusses current theories for detecting DRFM jamming and their merits. Chapter 4 will introduce the proposed EP technique, and Chapter 5 presents the results of simulations of the proposed EP technique and discusses its effectiveness. Finally, Chapter 6 summarizes the work and proposes topics for future research. 


\section{Chapter 2}

\section{Review of Relevant Theory}

\subsection{Digital Radio Frequency Memory Jammers}

In the recent past the use of coherent radars employing Doppler and pulse compression techniques has required jammers to overcome substantial processing gains of $30 \mathrm{~dB}$ to $60 \mathrm{~dB}[2]$. This means that jammers are no longer able to simply use pre-programmed waveforms against modern radar. They need to use more accurate replicas of radar waveforms to counter the processing gains of coherent radar. Enter the Direct Radio Frequency Memory (DRFM) jammer. The DRFM allows for the storage of intercepted radar signatures in a digital memory to allow for EA processing and subsequent transmission back to the radar.

\subsubsection{Architecture}

There are two main types of DRFM jammer architectures: wideband and multiple narrowband [2]. A wideband DRFM requires high-end ADCs and DACs and operates at high speed, while the multiple narrowband DRFM uses many cheap, low speed ADCs and DACs. Each could be designed to cover the same operational bandwidth but the wideband DRFM will have a superior instantaneous bandwidth.

The multiple narrowband DRFM has the advantage of being cheaper, the ability 
to use higher bit converters, and it is less complex to implement. This is due to the fact that each DRFM can be tuned to its own threat. This allows for easy segregation and reduced intermodulation between signals that cause spurious components in the received signal [2]. The disadvantage of this architecture is that it is generally not capable of countering frequency agile radars or pulse compression radar waveforms that exceed its limited instantaneous bandwidth.

The advantage of the wideband architecture is that it can replicate any radar signal in its frequency range, including frequency agile and pulse compression radars. Though it is more expensive and more complex, most research and development is directed toward wideband DRFMs.

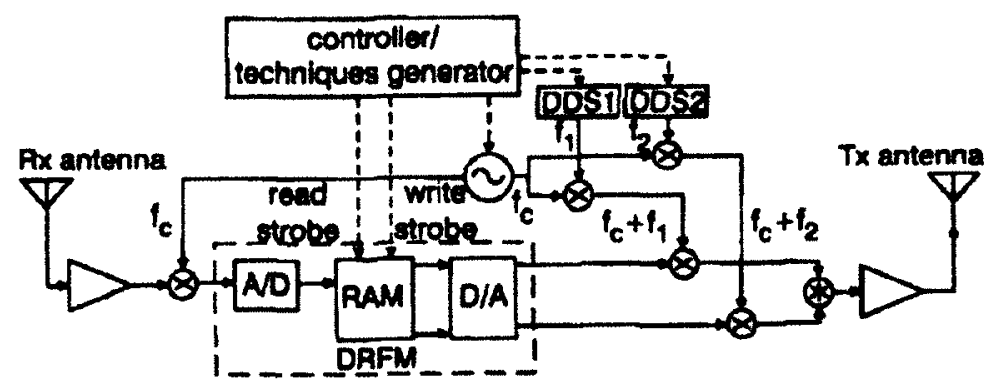

Figure 2.1: Block Diagram of Generic DRFM Jammer [5]

Figure 2.1 shows a block diagram of a generic DRFM jammer. It shows a single DRFM or wideband architecture. However, the same architecture could be used several times with some added control logic to create a multiple parallel narrowband architecture.

\subsubsection{Jamming Techniques}

Jamming, part of EA, has 2 main types, noise and deception. While the DRFM jammer can be used in both ways, the DRFM out performs all other types of jammers in deception techniques. This section will therefore focus on the common deception jamming techniques employed by DRFM jammers: Range Gate Pull-off (RGPO), 
Velocity Gate Pull-off (VGPO), cross-eye, smart noise and multiple pulse repeat. Detailed descriptions of these techniques can be found in [5] and [6], with only short descriptions found below.

RGPO is also known as range gate stealing or range gate walk-off. The purpose of RGPO is to create a false target in the threat radar that is offset in range, or equivalently time, from the actual skin return [5]. It can create down range and up range targets, the latter being the most difficult. A DRFM jammer is able to do this by delaying the retransmission of a replica waveform repeatedly (after each received pulse) for down range targets, or preemptively transmitting replica waveforms repeatedly for up range targets. The ultimate goal of the RGPO technique is to cause break-lock in the radar tracker.

VGPO can also be known as velocity gate stealing or velocity gate walk-off. The purpose of VGPO is to create a false target in the threat radar that is offset in velocity, or equivalently Doppler, from the actual skin return [5]. This technique is typically varied in time so that it seems that the created false target is "pulled-off" the skin return. A DRFM jammer is able to achieve this by retransmitting a replica of the waveform with an added Doppler shift. Again, the ultimate goal is to cause break-lock in the radar tracker.

RGPO and VGPO can be coordinated together. This ensures that the range history is consistent with the velocities portrayed by the jammer [5]. If these techniques are not coordinated, radars with both range and range rate trackers can detect the physically impossible motions, and then deduce those signals to be from a jammer.

Cross-eye jamming is an angular deception technique. This technique's goal is to create a false target in the threat radar that is offset in angular bearing from the actual skin return. The false target is created through interference between two signals. By design, the interference that is carefully manufactured by the jammer distorts the phase front and, in consequence, the apparent direction to the target [7]. 
This technique requires two antennae to be spaced fairly far apart from each other on an axis which is close to perpendicular to the boresight of the radar to be jammed. When a pulse is received, it is recorded from both antennae. It is then repeated back to the target from the opposite antenna. The signals are also transmitted out of phase when compared to each other. This is what causes a distorted phase front. This technique "steers" the threat away with the ultimate goal of causing break-lock.

Smart noise, sometimes referred to as a cover pulse, is band limited white noise which is usually centered on the Doppler frequency and range bin of the skin return [5]. This technique is typically used to mask the true target or to cause break-lock.

Multiple pulse repeat is used to create multiple false targets. It does this by repeating multiple waveform replicas within the radars Pulse Repetition Interval (PRI) [5]. This can mask the true target, overload a radar tracker, cause break lock or simply confuse an operator.

\subsection{Digital Signal Processing (DSP)}

The industry preferred domain for signal processing is now the digital domain. In equipment that uses signal processing, such as radars, designers typically try to convert from the analog to digital domain as early as possible after the reception of signals, and as late as possible before the transmission of signals. This is due to the flexibility of software, the ever increasing power of DSP processors and Field Programmable Gate Arrays (FPGAs), increasing memory density, decreasing size, decreasing power requirements and the ability to replace large and finicky wave guides with simple wires. For these reasons, all processing in the EP technique to be introduced in this thesis will use DSP and specifically will use the elementary DSP topics briefly introduced below. 


\subsubsection{Sampling Theorem}

Sampling is the foundation upon which DSP is built. This is the first, and arguably the most important step in the analog-to-digital conversion process. Sampling serves to convert a continuous-time signal to a discrete-time signal by taking "samples" on the continuous-time signal at discrete-time instants [8]. Quantization and coding account for the remaining steps of the analog-to-digital conversion process. These steps convert the discrete-time continuous-valued signal to a discrete-valued signal, and then represent each discrete value as a $b$-bit binary sequence. While these steps are important, this thesis will focus mainly on sampling.

The sampling theorem is used to select a sampling period $T_{s}$ or, equivalently, a sampling frequency $f_{s}$, to allow for proper conversion from continuous-time to discrete-time. The sampling theorem requires some basic information about the frequency content of a signal to be sampled in order to choose $f_{s}$. In most cases, knowing the maximum frequency, $F_{\max }$, of a signal is all that is required. From Nyquist we know that the highest frequency in an analog signal that can be unambiguously reconstructed is $\frac{f_{s}}{2}$ when sampled at $f_{s}$. If the signal being sampled contains frequencies above $\frac{f_{s}}{2}$ or below $-\frac{f_{s}}{2}$, those frequencies will erroneously appear in the range, $-\frac{f_{s}}{2}<f<\frac{f_{s}}{2}$. This is referred to as aliasing. To avoid aliasing, $f_{s}$ is selected such that

$$
f_{s}>2 F_{\max }
$$

and a filter is applied before the ADC to ensure that extraneous signal components, such as noise, higher than $F_{\max }$ are removed.

The sampling theorem states [8]:

If the highest frequency contained in an analog signal $x_{a}(t)$ is $F_{\max }=B$ and the signal is sampled at a rate $f_{s}>2 F_{\max } \equiv 2 B$, then $x_{a}(t)$ can be 
exactly recovered from its sample values using the interpolation function

$$
g(t)=\frac{\sin 2 \pi B t}{2 \pi B t}
$$

Thus $x_{a}(t)$ may be expressed as

$$
x_{a}(t)=\sum_{n=-\infty}^{\infty} x_{a}\left(\frac{n}{f_{s}}\right) g\left(t-\frac{n}{f_{s}}\right)
$$

where $x_{a}\left(\frac{n}{f_{s}}\right)=x_{a}(n T) \equiv x(n)$ are the samples of $x_{a}(t)$.

In ideal conditions, sampling at $f_{s}$ will allow exact reconstruction of the original continuous-time signal. However, in practice, this is not possible for two primary reasons. First, Equation (2.3) requires an infinite amount of samples. Second, it is assumed that signals at frequency at or close to $\frac{f_{s}}{2}$ are sampled at their ideal phase.

An illustration of the latter is given in Example 1.4.3 of [8]:

Consider the analog signal:

$$
x_{a}(t)=3 \cos 50 \pi t+10 \sin 300 \pi t-\cos 100 \pi t
$$

sampled at the Nyquist rate, $f_{s}=300 \mathrm{~Hz}$. The frequencies present in $x_{a}(t)$ are $25 \mathrm{~Hz}, 150 \mathrm{~Hz}$ and $50 \mathrm{~Hz}$ in order of the terms in (2.4). It should be observed that the signal component of $10 \sin 300 \pi t$, sampled at the Nyquist rate, results in samples at $10 \sin \pi n$, which are identically zero. In other words, we are sampling the analog sinusoid at its zero-crossing points. This problem would not occur if the sinusoid is offset in phase by some amount $\theta$. If $\theta \neq 0$ or $\pi$, the samples taken at the Nyquist rate are not all zero. However, we still cannot obtain the correct amplitude from the samples when the phase $\theta$ is unknown. 
When $\theta$ is unknown the sampling is incoherent. Therefore, the complete reconstruction of a continuous-time signal cannot be guaranteed, especially for frequency components close to the Nyquist frequency. Chapter 4 will capitalize on this fact.

\subsubsection{Digital Finite Impulse Response (FIR) Filters}

Digital FIR filters are a basic building block of DSP. An FIR filter changes an input $x(n)$, based on its impulse response $h(n)$ to produce the output $y(n)$. It is referred to as finite because removing an input causes the output to return to zero after a finite number of samples, $M$. This makes the FIR filter more stable and easier to design compared to an Infinite Impulse Response (IIR) filter. The trade-off is that FIR filters typically require more processing time. From [8] we have two descriptions of the generic FIR filter: the convolution formula (2.5), and the difference equation (2.6) which show the relationship between the output and input.

$$
\begin{gathered}
y(n)=\sum_{k=0}^{M-1} h(k) x(n-k) \\
y(n)=b_{0} x(n)+b_{1} x(n-1)+\ldots+b_{M-1} x(n-M+1) \\
=\sum_{l=0}^{M-1} b_{l} x(n-l)
\end{gathered}
$$

In (2.6), $b_{l}$ represents the filter coefficients and in the case of FIR filters

$$
b_{l}=h(l), l=0,1, \ldots, M-1
$$

Since these coefficients are simply stored in memory and accessed by the DSP processor when required they can be changed easily, thereby changing the characteristics of the filter. This capability will be used in Chapter 4 to create a new filter for each 
radar pulse.

\subsection{Matched Filtering}

Typically, maximizing the Signal-to-Noise Ratio (SNR) at the radar receiver maximizes the detectability of a target [9]. Therefore, it is a fundamental goal in radar design to maximize the SNR as it can impact all aspects of the radar including transmitter power, antenna size, DSP speed, etc [10]. Matched filtering is one of the ways of accomplishing this goal and is widely used in radar signal processing. A matched filter is designed have a maximal response to, and only to, the signal for which it was designed. In the case of radar, the radar pulse, and it is able to do so when the received signal has significant noise interference. In fact, the matched filter is the optimum filter for non-fluctuating radar targets [10]. There are many techniques to derive the matched filter, in the time or frequency domains, that will not be shown here but can be found in [9] and [10]. The result is an impulse response that is dependent on the original signal. The impulse response of a matched filter from [10] is:

$$
h(t)=k s^{*}\left(T_{M}-t\right)
$$

where $\mathrm{k}$ is a constant, $s^{*}$ is the complex conjugate of the originally transmitted signal, and $T_{M}-t$ indicates that the signal $s^{*}$ is reversed in time. Therefore, a radar simply needs to keep a copy of the transmitted signal, take its complex conjugate, reverse it in time and then use the results as the coefficients for an FIR filter in order to implement a matched filter in the radar receiver.

Taking (2.8) to the digital domain we simply have:

$$
h(n)=k s^{*}\left(N_{M}-n\right)
$$


which is the impulse response of the matched filter. Using (2.7) it becomes trivial to define the filter coefficients, $b_{l}$, to implement a digital FIR filter as the required matched filter:

$$
b_{l}=k s^{*}\left(N_{M}-l\right), 0<l<N_{M}-1
$$

\subsection{Integration}

As stated in Section 2.3, maximizing the SNR at the radar receiver is a fundamental goal of radar design. Another tool that a radar designer uses to do this is integration. Unlike matched filtering which is used on a single radar pulse, integration acts on multiple radar pulses. However, integration can be used in conjunction with matched filtering. Integration is the process of combining multiple samples of a pulse, each with noise or other types of interference, in order to "average out" the noise and subsequently have a combined sample with a significantly higher SNR than the individual pulse samples. There are three main types of integration: coherent, non-coherent, and binary. The two types of interest for this thesis are non-coherent and binary, each of which will be introduced below.

\subsubsection{Non-coherent Integration}

Non-coherent integration does not use phase information to perform integration. A linear non-coherent detector is defined in [10] as:

$$
|x|=\sum_{n=0}^{N-1}|x[n]+w[n]|
$$

where $x[n]$ is the signal to be detected and $w[n]$ is noise. There are other types of non-coherent integration detectors including square law and logarithmic. The square 
law detector is possibly the most common and [10] defines it as:

$$
|x|^{2}=\sum_{n=0}^{N-1}|x[n]+w[n]|^{2}
$$

The result of this integration of $N$ pulses will be a higher SNR than for an individual pulse. The magnitude of the SNR increase will be based on the particular statistics of the interference.

\subsubsection{Binary Integration}

The binary integration method was the first method developed to make a detection decision without an operator [9]. While it is not as efficient as coherent or non-coherent integration it is still an important technique because it is simple to implement. As a radar antenna scans a target it would ideally receive $n$ returns from the target. However, due to interference it will typically receive fewer than $n$ returns. This integration technique simply counts the number of actual returns and compares this to a threshold $m$. If greater than $m$ returns are received, a target is declared present. If less than $m$ returns are received, a target is not declared present. Detectors employing this technique are usually referred to as binary detectors, or $m$ of $n$ detectors. This method of integration commonly follows coherent or non-coherent integration in a multi-stage integration scheme.

\subsection{Random Variables}

The domain of EW and radar is fraught with chaotic systems, from noisy signals, to erratic radar cross section fluctuations, to the geometry of engagements. None of these are deterministic processes, they are random or stochastic processes. The radar and other EW equipment must be able to cope with this chaos and still reliably 
complete its task. At the heart of random processes are random variables.

A random variable $X$ is a function that assigns a real number, $X(\zeta)$, to each outcome $\zeta$ in the sample space of a random experiment [11]. Figure 2.2 shows this

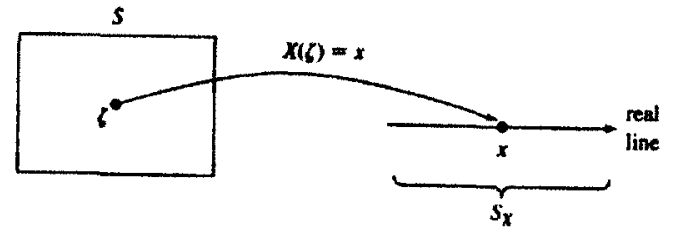

Figure 2.2: Random Variable Mapping [11]

pictorially, where $S$ is the sample space and domain of $X$, and the set $S_{X}$ of all values taken on by $X$ is the range of the random variable. The notation commonly used is upper case letters for random variables and lower case letters for possible values of the random variables.

In most cases there is a priori knowledge, though usually limited, to determine the likelihood that $X(\zeta)$ will take on the value $x$. The most useful representation of this knowledge is the Probability Density Function (PDF). The PDF of $X$ is denoted $f_{X}(x)$. With knowledge of the PDFs of random variables, designers are able to model the chaos and develop systems to overcome it.

It is not uncommon to have problems with more than one random variable at play, as will occur in this thesis. In the case of this thesis, it is required to add two random variables to yield a third. From [11], the equation for the PDF of $Z=X+Y$ is given by:

$$
f_{Z}(z)=f_{X}(x) * f_{Y}(y)
$$

when $X$ and $Y$ are independent random variables. 


\section{Chapter 3}

\section{Current EP Techniques Countering DRFM Jammers}

This chapter will explore the existing research and development in the field of EP against DRFM jammers. The focus will be on current techniques to counter deception jamming - the niche of the DRFM jammer. The current research on EP techniques to counter DRFMs can be sub-divided into 2 types: (1)separation and filtering and (2)denial.

The first type of technique is meant to separate target returns from DRFM returns in space, time and/or frequency which allows the unwanted signal to be filtered out. Most of the work presented provides fairly robust techniques for separating target and DRFM signals. However, the most common shortcoming is in the determination of whether or not the second return is actually from a jammer. Most techniques make assumptions that if returns are close enough in space or time, and the second target has greater amplitude, then they must be target and jammer returns respectively. However, aircraft are able to fly in formation, only meters apart, and can have significant variations in RCS while maneuvering, which can cause changes in amplitude. Misidentifying an enemy aircraft as a jammer and filtering out its return could have disastrous effects. 
The second type of technique attempts to deny the DRFM from making useful recordings of radar pulses. There are two different techniques proposed to accomplish this. The first has the goal of making any recorded signals obsolete before the DRFM can repeat the signal back to the radar. Pulse-to-pulse diversity allows for this if the assumption is made that the DRFM must wait until the next radar pulse to make use of its current recorded pulse. The second technique is to use a random noise waveform. This could be characterized as "sample-to-sample diversity", since every sample at the ADC of the DRFM will be random. The assumption made for this technique is that the DRFM must have a throughput delay approaching 0 ns [4] and therefore, the DRFM is forced to predict and transmit, rather than record and transmit. The current shortest throughput delays are $25 \mathrm{~ns}$ (or $7.5 \mathrm{~m}$ ). While this may be unacceptable for DRFMs attempting to conceal small aircraft, it may be acceptable for protecting large targets such as naval ships. As discussed in Section 2.1.2, the DRFMs most commonly used jamming techniques are RGPO, VGPO and cross-eye. A DRFM type jammer is not a prerequisite for these jamming techniques but the use of a DRFM greatly increases their effectiveness. In the following discussions of existing research, it is obvious that a great deal of research has been completed and made available in open literature for RGPO, but little is available on VGPO and cross-eye. In order to summarize concisely, Section 3.3 contains a summary table matching the discussed current EP techniques to jamming techniques.

\subsection{Separation and Filtering}

The following sub-sections present current research on EP techniques designed to identify and separate DRFM returns from target returns. The techniques are: stretch processing, rapid relock, narrow gate monitoring and frequency diversity. Stretch processing includes low pass filtering to filter out the DRFM return and the Rapid 
Relock and Narrow Gate Monitoring methods filter out the DRFM return through gating. The research on frequency diversity does not currently offer a filtering scheme.

\subsubsection{Stretch Processing}

Stretch processing is often used in radars to increase range resolution in LFM radars without decreasing the pulse width of the radar. It has also been demonstrated as a potential technique to detect and reject DRFM jammer signals in [12]. In stretch processing a transmit signal with LFM of high bandwidth is used, and unlike typical pulse compression radars, the Local Oscillator ( $\mathrm{LO}$ ) is also swept during the receive period. The LO is mixed with the received signal and the result is the segregation in frequency of returns with similar ranges [13]. Figure 3.1 shows an example of stretch processing on a single target with three back scatterers.

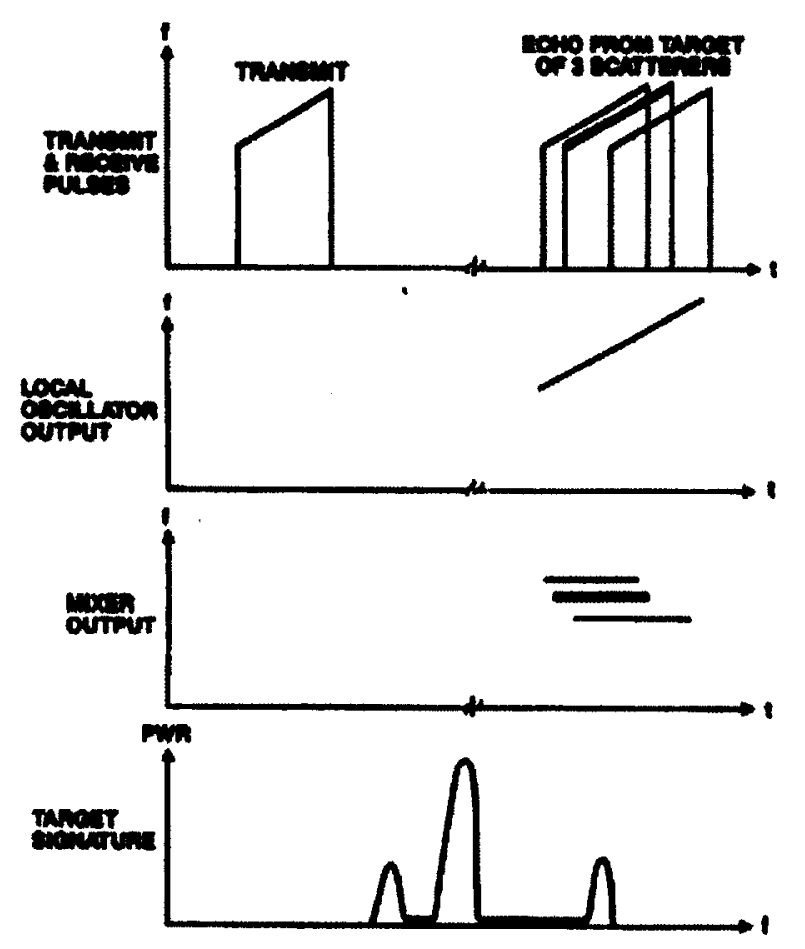

Figure 3.1: Stretch Waveform and Processing' [14] 
A DRFM jammer repeats the radar pulse with a certain delay. Therefore another plausible scenario for the top graph of Figure 3.1 is two scatters and a DRFM signalthe latest echo being the DRFM. To use this as an EP technique, one simply needs to set-up a low pass filter which, using the Figure 3.1 example, would filter out the highest frequency component shown in the bottom graph. The EP technique and the mathematics defining an appropriate cut-off frequency is presented in [12].

There are two limitations to this EP technique. Stretch processing is also used to distinguish between two different but adjacent targets, such as aircraft flying in formation. This leads to the first limitation of this EP technique, it could mistake two cooperative adjacent targets as one target with a jammer and start filtering out the return of the more distant target. In this scenario, the more distant target could then start jamming and the victim radar would detect this as a new target. The second limitation is that this technique only works with radars using LFM pulses.

\subsubsection{Rapid Relock}

A basic counter to jammers employing RGPO and VGPO exists by sensing abnormally large range rates or range accelerations in the targets track [15]. Most radars have a "rapid relock" function that, once jamming has been detected, attempts to relock the actual target based on its track before the RGPO or VGPO was detected. However, this technique is not robust. It simply forces the jammer to execute RGPO and VGPO in a coordinated fashion and assure that any movements of the target the jammer is suggesting are realistic for the host platform. Another difficulty will be reliably detecting that jamming is occurring. 


\subsubsection{Narrow Gate Monitoring}

Leading edge tracking is a simple EP technique used by tracking radars against RGPO. As explained in [15], this technique takes advantage of two characteristics of a simple RGPO. First, because of the RGPO's finite response time, the earliest the RGPO's pulse can arrive is just after the leading edge of the skin return. Second, simple RGPO's will usually pull the tracking gate to greater ranges. Therefore, the RGPO can be prevented from capturing the tracking gate by:

1. passing the receiver's video output through a differentiation circuit to provide a sharp spike at the skin return's leading edge,

2. narrowing the tracking gate, and

3. locking the gate onto the spike.

This is illustrated in Figure 3.2.

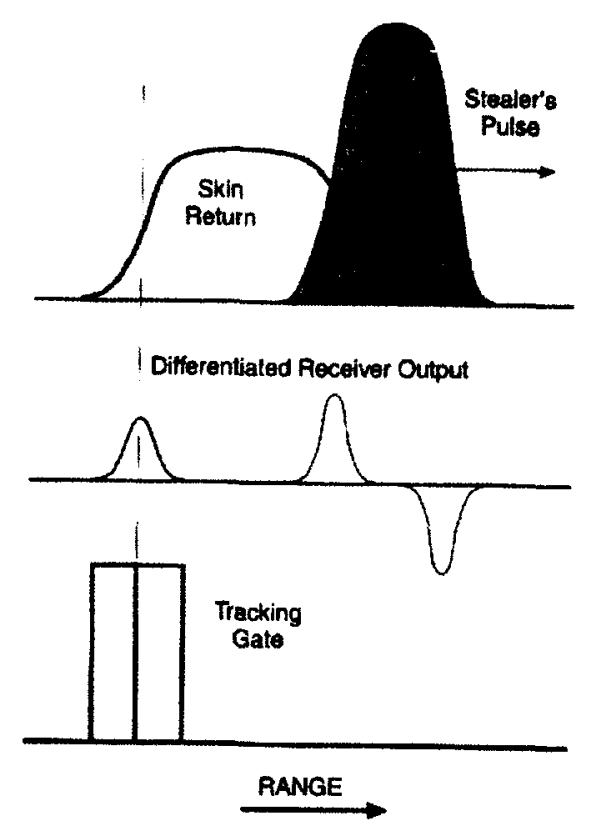

Figure 3.2: Simple Leading Edge Tracking [15] 
As throughput delay in DRFMs decrease, the ability to perform leading edge tracking becomes more difficult for radars with longer pulses. Using high rangeresolution monopulse tracking can mitigate this and provide an increased level of effectiveness to this technique, simply by ensuring you can capture the leading edge and by having a narrower tracking gate [16].

According to [17], there are two limitations to this technique. First, it can only defend against RGPO if the pull-off is done down range. A jammer capable of up range RGPO would fully defeat this technique. Second, the SNR needs to be high since the differentiation will cause SNR loss. If SNR is too low it is possible that the target will be omitted by Continuous False Alarm Rate (CFAR).

In order to remove these limitations, [17] modifies the above technique calling it Narrow Gate Monitoring. First, the concept of head and tail tracking is introduced to allow the new technique to operate against RGPOs up and down range. Second, a more complex gate arrangement and four stages of operation are defined to monitor the RGPO as it progresses, and adapt the gating strategy based on the progress of the RGPO. The four stages are illustrated in Figure 3.3 and listed below:

1. Recognizing the RGPO interference (via Automatic Gain Control (AGC) monitoring),

2. Normal tracking and monitoring the interval gates simultaneously until the jamming signal enters into the interval gates,

3. Head tracking (or tail tracking) and monitoring the edge gates simultaneously until the jamming signal enters into the edge gates, and

4. Normal tracking after the jamming signal is out of the whole gate.

Figure 3.3 gives an example of the progression of the four stages, showing the location of the target and the jamming in the gate arrangement. Progressing from stage 2 to 


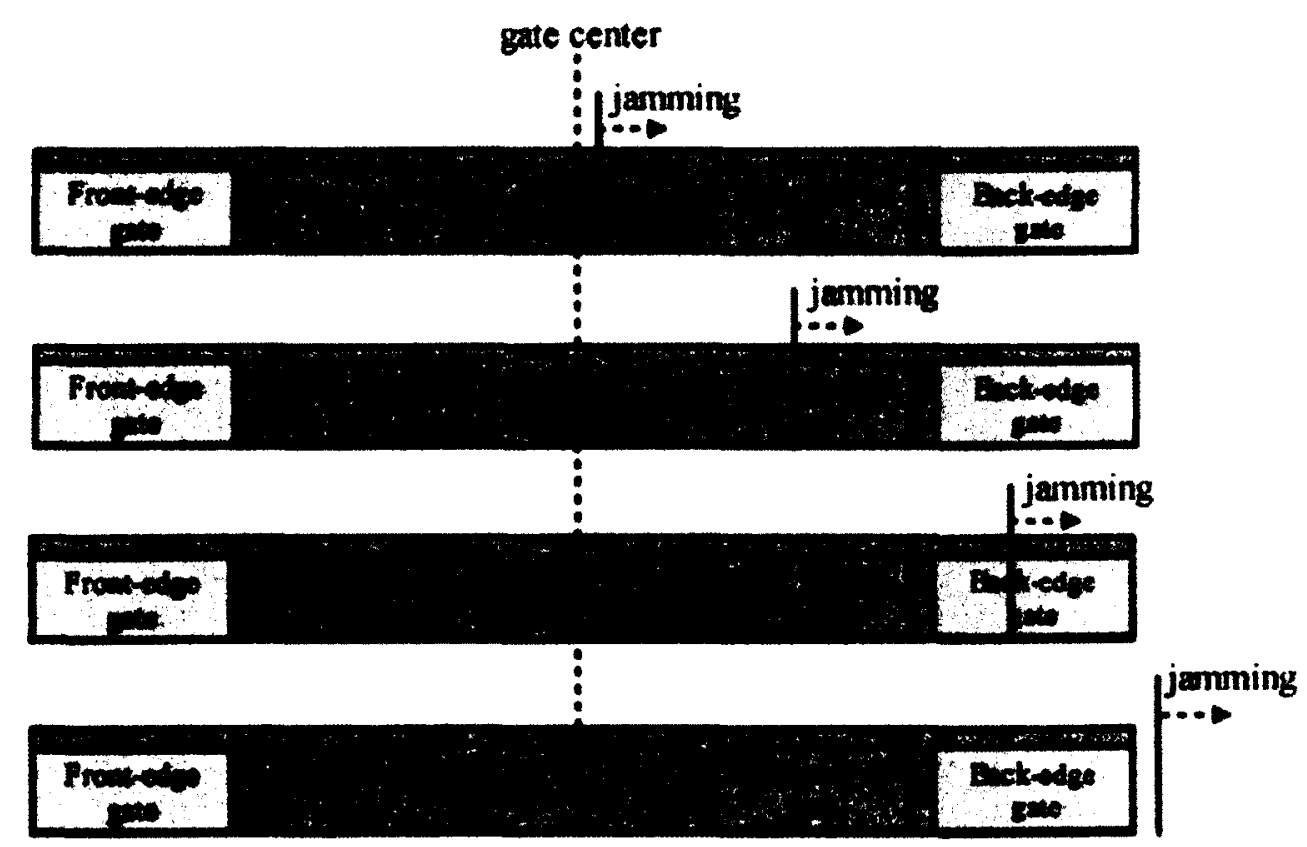

Figure 3.3: Example of Narrow Gate Monitoring [17]

3 is triggered when the absolute value of the difference between the front and back interval gate voltages breaks a threshold, which happens when the jamming enters one of the interval gates. The sign of the difference will determine whether head or tail tracking is to be used. Progressing from stage 3 to 4 is triggered by monitoring the edge gates in the same way as the interval gates.

No statistics concerning the effectiveness of this technique were provided, though it seems quite robust. Its limitation is that it can only counter the RGPO, can only be used by a tracking radar, and detection by AGC may be difficult if the DRFM attempts to transmit its signal only 1 to $2 \mathrm{~dB}$ above the skin return. 


\subsubsection{Frequency Diversity}

In their paper entitled "Discrimination of Targets and RGPO Echoes Using Frequency Diversity" [18], Blair and Brandt-Pearce present an EP technique that considers target echo sub-pulse amplitudes to be Rayleigh distributed, while the sub-pulse amplitudes of RGPO echoes are considered to be fixed across the frequencies. The paper assumes the radar is monopulse using pulse compression with $\mathrm{N}$ sub-pulses of distinct frequencies. The radar utilizes aperiodic revisit times and single-pulse dwells, and the jammer is a DRFM employing a RGPO technique. Estimators of the amplitude parameters for Rayleigh and fixed amplitude targets were developed and then used with a Generalized Maximum Likelihood (GML) and variance test to discriminate between Rayleigh and fixed amplitude echoes and therefore, between target and DRFM jammer returns. The technique was simulated with reliable results. Using 4 subpulses at SNRs greater than $10 \mathrm{~dB}$ provides a probability of discriminating a target as fixed amplitude of $P_{D F A}=0.58$ and with a false alarm rate of $P_{F D F A}=0.05$. With 4 pulses at $13 \mathrm{~dB}$ the results improve to $P_{D F A}=0.82$ and $P_{F D F A}=0.05$.

While this technique provides reliable results, it was only demonstrated for monopulse radar against the RGPO technique. Also, it may be possible for a jammer to attempt to imitate a Rayleigh target by purposefully changing amplitudes of sub-pulses before transmission.

\subsection{Denial}

The following sub-sections present current research on EP techniques designed to deny a DRFM the ability to operate effectively, rather than just detecting, separating and filtering the DRFM return. The techniques are pulse diversity and ultrawideband bandlimited random noise waveforms. 


\subsubsection{Pulse Diversity}

Radar systems using pulse diversity as an EP technique vary their transmitted pulses to force the jammer to analyse and record each incoming pulse. In [19] such a technique is proposed for Synthetic Aperture Radars (SAR) with the assumption that the jammer is always one pulse behind the radar. In other words, at slow-time position $n$, the radar is transmitting pulse $p_{n}(t)$ while the jammer will utilize $p_{n-1}(t)$ against the radar. To maximize the efficiency of this technique, $p_{n}(t)$ should be orthogonal to $p_{n-1}(t)$. Then the returns from the true target and the jammer are easily separated through matched filtering. In this case the matched filter is already part of the radar and has not been added solely for EP purposes. Hence, pulse diversity being located in the Denial section However, imposing the restriction of orthogonal pulses on the radar may cause problems for the radar in its primary role, such as imaging in this case. In the approach in [19], nearly-orthogonal pulses are used to allow for EP and to minimize the consequences on the radar's primary role. The technique is simulated to prove its utility, however no statistics on effectiveness were offered.

The assumption that the jammer is always a pulse behind the radar limits the effectiveness of this technique. This technique will not allow a jammer to spoof uprange targets, unless the jammer can predict future waveforms, but the possibility of recording $p_{n}(t)$ and repeating it in the same slow-time interval is possible. The current lower limit on DRFM jammer throughput delay is 25 ns as found in open literature [4]. Since radar pulses are typically longer than $25 \mathrm{~ns}$, a jammer would need to simultaneously receive and transmit in order to achieve a $25 \mathrm{~ns}$ throughput delay. According to [20], the Russian built Sorbitsiya-S jammer has four antennae, two on each wing-tip, one fore, one aft, and is capable of simultaneous receive and transmit using two same-facing antennas. Therefore, this technique would not be effective when countering all jammers. 


\subsubsection{Ultrawideband Bandlimited Random Noise Waveforms}

Imaging radars, most commonly SARs, typically use LFM pulses. The disadvantage is this waveform is quite predictable and consequently, simple for a DRFM jammer to replicate. Garmatyuk and Narayanan propose using ultrawideband (UWB) bandlimited random noise (BLRN) pulses rather than LFM pulses to counter DRFM jammers in [21]. They represent the stochastic UWB signal $P(t)$ as

$$
P(t)=A(t) \cdot \sin \left(\omega_{0} t+\phi_{0}(t)\right)
$$

where $A(t)$ is Rayleigh-distributed amplitude, $\omega_{0}$ is the central frequency, and $\phi_{0}(t)$ is uniformly distributed random phase. Given the 2-dimensional target function as $\zeta(x, y)$, where $x$ and $y$ are spatial coordinates of the target elements, then the radar return $\hat{s}$ is given by:

$$
\hat{s}(u, t)=P(t) \otimes \zeta(t)+J(t)
$$

where $u$ is the slow-time domain coordinate, $\zeta(t)$ is the target reflectivity function and $J(t)$ is a jammer signal. Then after correlation processing, the signal fed to the $\mathrm{I} / \mathrm{Q}$ detector is:

$$
s_{c o r r}=\hat{s}(u, t) \otimes P(t)=[P(t) \otimes \zeta(t)] \otimes P(t)+J(t) \otimes P(t)
$$

Simulations were conducted on the isolated $J(t) \otimes P(t)$ term with the results shown in Figure 3.4. The simulations were conducted with an LFM waveform and the BLRN waveform, $P(t)$. Figure 3.4(a) shows the radar produced LFM and the jammer predicted signal, while Figure 3.4(b) shows the BLRN waveform along with the jammer predicted signal. Finally, Figure 3.4(c) shows the normalized cross-correlation functions between the jammer predicted and real waveforms. The BLRN waveform severely reduces the effectiveness of the jammer compared to the LFM waveform. 
However, to arrive at this result it was assumed that the jammer was attempting to

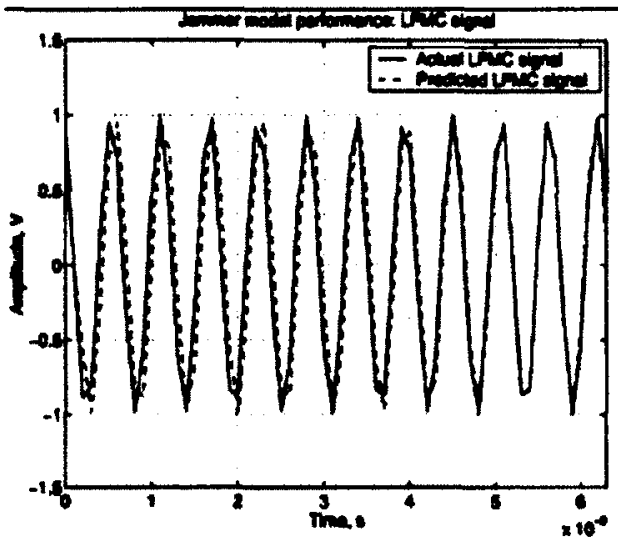

(a)

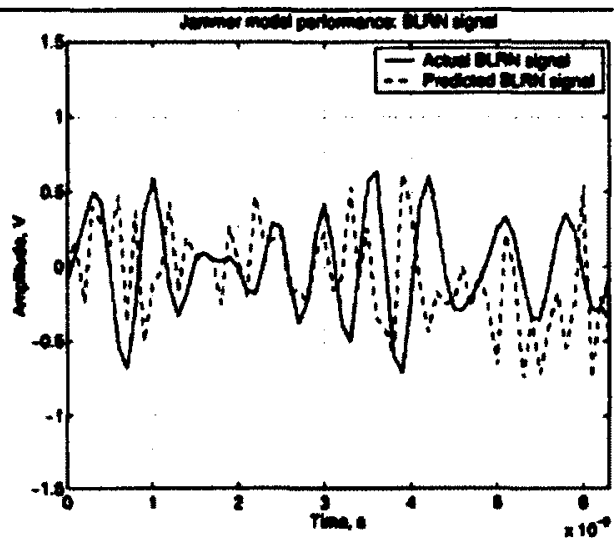

(b)

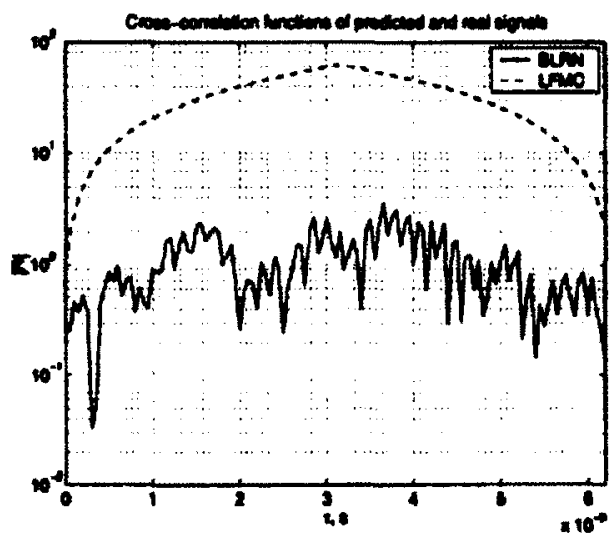

(c)

Figure 3.4: Jammer Model Performance. (a) Predicted LFM chirp signal, (b) Predicted BLRN signal, (c) Cross-correlation functions of predicted and real radar signals [21]

predict the radars instantaneous frequency and adjust its own instantaneous frequency based on past recorded radar pulses. This assumption ensures that the jamming signal could completely mask the target in the image. However, this makes the jammer's task much more difficult in that it must have a throughput delay of $0 \mathrm{~s}$. DRFM jammers with throughput delays less than $25 \mathrm{~ns}$ can be found in open literature [4]. Such a delay translates into $7.5 \mathrm{~m}$, which may be an unacceptable delay for small targets but may be acceptable for large targets or for jamming space based imaging radars. 
Allowing a DRFM jammer to record and repeat a signal with a minimum throughput delay could hamper the effectiveness of this EP technique.

This technique has been theoretically proven for other radar applications such as doppler estimation, inverse SAR (ISAR) and in phase comparison monopulse [22]. This technique provides EP benefits to all of these applications but it also causes those radars to not perform as well in their primary role since they must abandon the waveform tailored to their primary role (i.e. LFM) and use the BLRN waveform. The concept of using a BLRN waveform will be used in Chapter 4, but in a technique that allows the radar to retain the use of its tailored waveform.

\subsection{Summary}

Table 3.1 provides a summary of the current research on EP techniques countering DRFMs. It shows the radar type targeted by each technique, the jamming countered and potential problems with the technique. As can be seen from this literature review, there are two main areas lacking research in open literature. They are (1) reliable detection of DRFM returns and (2) ability to counter DRFMs, without assuming they are a pulse behind the radar. The next chapter presents a novel EP technique designed to address these issues. 
Table 3.1: Summary of Current EP Techniques countering DRFMs

\begin{tabular}{|c|c|c|c|}
\hline EP Technique & Radar Type & $\begin{array}{l}\text { Jamming } \\
\text { Countered }\end{array}$ & $\begin{array}{l}\text { Possible Problems with EP } \\
\text { Technique }\end{array}$ \\
\hline Stretch Processing & Radars using LFM & Any & $\begin{array}{l}\text { Determining if a return is real or } \\
\text { jammer }\end{array}$ \\
\hline Target Tracking & Tracking & RGPO, VGPO & $\begin{array}{l}\text { Defeated by realistic pull-off pro- } \\
\text { gramming }\end{array}$ \\
\hline $\begin{array}{l}\text { Narrow Gate Moni- } \\
\text { toring }\end{array}$ & Tracking & $\begin{array}{l}\text { RGPO (VGPO, } \\
\text { conceivably) }\end{array}$ & $\begin{array}{l}\text { Determining if a return is real or } \\
\text { jammer }\end{array}$ \\
\hline $\begin{array}{l}\text { Frequency Diver- } \\
\text { sity }\end{array}$ & $\begin{array}{lr}\text { Monopulse } & \text { (pulse } \\
\text { compression } & \text { with } \\
\text { N sub-pulses) }\end{array}$ & RGPO & Limited to specified radar type \\
\hline Pulse Diversity & $\begin{array}{l}\text { SAR (others, con- } \\
\text { ceivably) }\end{array}$ & Any & $\begin{array}{l}\text { Assumes that jammer is a pulse } \\
\text { behind the radar }\end{array}$ \\
\hline $\begin{array}{l}\text { Bandlimited Ran- } \\
\text { dom Noise }\end{array}$ & $\begin{array}{lr}\text { SAR, } & \text { ISAR, } \\
\text { Phase } & \text { Compar- } \\
\text { ison } & \text { Monopulse, } \\
\text { Doppler Estimation }\end{array}$ & Any & $\begin{array}{l}\text { Assumes jammer is either one } \\
\text { pulse behind or is predicting } \\
\text { rather than recording and trans- } \\
\text { mitting }\end{array}$ \\
\hline
\end{tabular}




\section{Chapter 4}

\section{Radar Electronic Protection using Concatenated Random Noise (CRN)}

This chapter will introduce an EP technique that exploits incoherent sampling at the jammer using Concatenated Random Noise (CRN). It uses both denial and separation, as explained in Chapter 3. The technique uses a short CRN pulse, strictly for EP purposes, that is designed to deny the DRFM the ability to properly record it. This CRN pulse will be concatenated to the regular radar pulse, thereby not affecting the radar's effectiveness in its primary role by allowing the radar to use its optimal waveform. The technique will process the returns of these CRN pulses separately from the radar receiver. Once the technique has received enough CRN pulses it will make a decision whether the target in question is in fact a target or a DRFM jammer and notify the radar tracker. The tracker could then simply drop that target track-the separation part of the technique.

The CRN technique is aimed to fill a void in the open literature in the following areas:

1. EP techniques that counter angular deception jamming such as cross-eye,

2. EP techniques that exploit a design feature of DRFM jammers that is not easily changed, 
3. EP techniques that make no assumptions on the throughput delay of the jammer,

4. EP techniques that assume the jammer can cope with pulse-to-pulse diversity,

5. EP techniques that use the contents of a replica pulse, rather than external information such as spatial, temporal, doppler or average power information, and

6. EP techniques to aid in reliable identification of jammer signals to ensure that existing jamming signal separation and filtering techniques, presented in Chapter 3 , are only used against jamming signals.

The technique borrows the idea of using a random noise signal from [21] and [22]. However, instead of assuming the jammer must predict the noise waveform and return it to the radar with a $0 \mathrm{~ns}$ throughput delay, this technique allows the DRFM to fully record the waveform and retransmit it, yet still be able to distinguish between the DRFM generated waveform and skin return.

It is foreseen that the proposed technique would be most beneficial for radars with a pulse duration in the tens of microseconds or higher. However, depending on the typical SNR values and the value of EP compared to other functions competing for radar resources, it may be beneficial for radars with lower pulse durations. In terms of Pulse Repetition Frequency (PRF), this technique will work most effectively on a Medium PRF (MPRF) radar, as will be shown in the rest of this Chapter, however it is a good approximation for High PRF (HPRF) radars as well, as shown in Appendix A. Furthermore, to simplify the problem space, an assumption was made to model fighter aircraft in order to be able to simplify random variables to uniform distributions. More research is required to determine if this technique would be equally effective in less dynamic environments such as land, maritime and space. 
In the rest of this chapter the CRN technique will be introduced, first at the conceptual level and then at a more detailed design level. The conceptual introduction will first explain and model the incoherent sampling phenomenon which will lead into a conceptual explanation of the CRN technique. The design level discussion will provide details on how the $\mathrm{CRN}$ technique could be implemented in a generic radar system and how it was simulated for this thesis.

\subsection{Concept}

The concept of the CRN technique is to exploit a characteristic of DRFM jammers that the designer cannot change-the use of an ADC and DAC. The CRN technique assumes that the radar and DRFM have comparable ADCs and DACs, both in sampling frequency and quantization bits. That is to say, it assumed that both the radar and DRFM designer will have access to comparable levels of technology. The CRN technique also uses two main facts. First, the signal path from radar through the jammer and back to the radar has an added ADC and DAC compared to the signal path of the true target. This is the key to distinguishing between the true target return and the DRFM jammer return. Second, the sampling of the DRFM and the radar receiver is incoherent with respect to the sampling of the radar transmitter. Both of these facts provide an opportunity for EP. This section will explain the incoherent sampling effect that causes sampling delay, find a PDF for the sampling delay and then briefly give a high-level description of the EP Processor, that would work in concert with the radar in order to implement the CRN technique.

\subsubsection{Sampling Delay}

Noise will be distorted by the extra ADC and DAC in the signal path, especially noise close to $\frac{f_{s}}{2}$. This is due to the fact that the DRFMs sampling is not, and cannot, be 
coherent with the sampling of the radar. In effect, the ADC of the DRFM adds a delay from the original transmitter sampling points to the DRFM sampling points. This delay is $\tau$, where $\tau$ is defined as:

$$
0<\tau<\frac{1}{f_{s}}, 0<\tau<T_{s}
$$

where $f_{s}$ is the sampling frequency and $T_{s}$ is the sampling period of the ADC. This effect is explained with a simple sinusoid example in Section 2.2.1 and its effects on random noise are shown in Figure 4.1 for $\tau=\frac{T_{n}}{2}$, the worst case delay. It can be

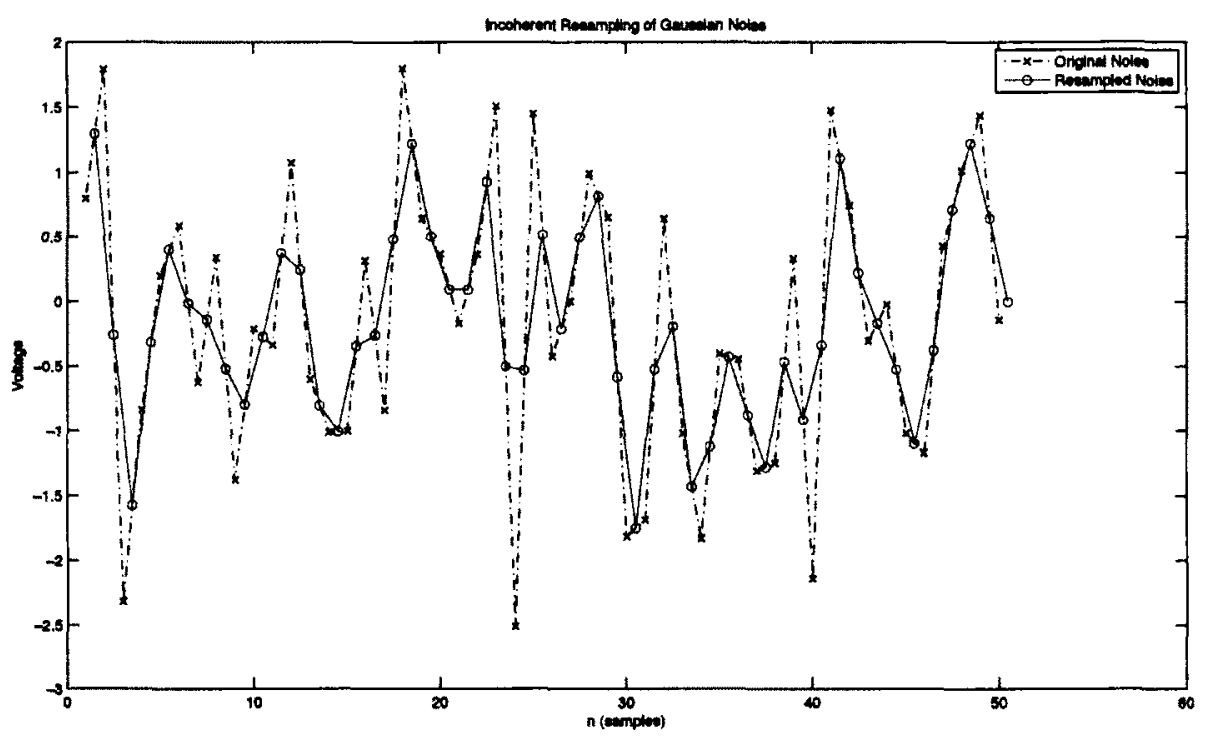

Figure 4.1: Example of Incoherent Sampling of Gaussian Noise

seen in Figure 4.1 that the signal is distorted since it is sampled with a delay of $\tau$. Figure 4.1 is simplified since it does not show interpolation that would happen after the $\mathrm{ADC}$, however, this approximation is used. It would be desirable to add realistic interpolation to the simulation in future research. If ideal ADCs, DACs and filters were used, this phenomenon would not occur due to Shannon's Law and the fact that the noise would be perfectly reconstructed through interpolation. However, this is 
not the case and the approximation of $\tau$ is used to simulate the incoherent sampling and imperfect interpolation. The radar-target slant range, rate of change of the slant range, sampling jitter and sampling frequency will all have effects on the value of $\tau$ from pulse to pulse. (Note: To allow for brevity, radar-target slant range, the range in three dimensions, will be referred to as range for the remainder of this chapter.) This thesis will assume the same sampling frequency used in [21], another random noise radar, in order to give concrete results rather than results based on normalized frequencies. Therefore, we use $f_{s}=6.67 \mathrm{GHz}$ or $T_{s}=150 \mathrm{ps}$. Using $\frac{c}{f_{s}}$ to calculate the distance between samples, where $c$ is the speed of light, we get $45 \mathrm{~mm}$. In order for a DRFM to sample coherently with the radar it would need to predict the range from the radar to target to a fraction of $45 \mathrm{~mm}$. It is clear that coherent sampling is impossible.

Furthermore, it is a valid assumption that $\tau$ is a random variable due to the nature of electronic warfare engagements. Typically the engagements are highly dynamic, most notably in aircraft versus aircraft, and aircraft versus missile engagements. To further qualify this statement, it is assumed that $\tau$ is a random variable pulse to pulse but $\tau$ is static within a pulse. In other words, within a pulse all samples will be delayed by the same value. In Section 4.1.2 a PDF is found for $\tau$ using a typical MPRF radar configuration, Appendix A shows the same derivation for a HPRF radar configuration. The MPRF configuration is assumed to have a PRF of $10 \mathrm{kHz}\left(T_{P R I}=100 \mu s\right)$ and HPRF to have a PRF of $100 \mathrm{kHz}\left(T_{P R I}=10 \mu s\right)$.

\subsubsection{PDF of Sampling Delay $\left(f_{\tau}(z)\right)$}

In order to properly simulate this sampling delay phenomenon we must find a PDF to define the random variable $\tau$. In order to explain this phenomenon, we will take the viewpoint of a receiver, either the radar or the DRFM. At the first reception of a signal originating from the radar transmitter there will be a random delay of $\tau$ 
between the original transmitter sampling points and the receiver sampling points, as demonstrated in Figure 4.1, where $\tau=\frac{T_{s}}{2}$. This delay will be constant for all samples in one pulse only. The generic value of $\tau_{q, p}$, where $p$ is the pulse number within a Coherent Pulse Interval (CPI) and $q$ is the CPI number, will be:

$$
\tau_{q, p}=R_{q}+p \cdot V_{q}
$$

Here we are assuming that the host radar uses a CPI and therefore, the CRN technique will adapt to use the same concept, since the EP pulses are sent with the radar pulses. The CRN technique does not actually require coherent processing, however, the term is borrowed since it is widely understood in terms of the radar's operation.

In Equation (4.2) $R_{q}$ is a random variable which contributes delay due to range, and $V_{q}$ is a random variable which contributes delay due to velocity or more specifically, the rate of change of range. Both $R_{q}$ and $V_{q}$ are static for the duration of CPI $q$. In essence, $R_{q}$ establishes the initial delay for CPI $q$ and $p \cdot V_{q}$ modifies the delay for each pulse in the CPI through the multiplication by $p$. The latter takes into account rate of change of the range, since both radar and target are typical moving at high speeds. It is assumed that the relative accelerations between the radar and target will cause little change in the relative velocity over the course of a CPI and therefore, accelerations are not considered in Equation (4.2). The duration of a CPI will be:

$$
T_{C P I}=N_{C P I} \cdot T_{P R I}
$$

where $N_{C P I}$ is the number of pulses or equivalently, the number of PRIs in a CPI, and $T_{P R I}$ is the pulse repetition interval. Their typical values are $N_{C P I}=10$ and $T_{P R I}=100 \mu s$ which yields $T_{C P I}=1 \mathrm{~ms}$. 
First, let us consider the PDF of $V_{q}, f_{V_{q}}(y)$. The value of $V_{q}$ will be most affected by the instantaneous velocities of the target and radar, the geometry of the engagement, and maneuvering of the radar and target. An example of the latter is an aircraft rolling; a wing pylon could be providing the strongest radar return and if the aircraft is rolling the distance between the radar and the wing pylon will be changing proportional to the roll rate. Properly modeling the physics of an engagement to determine precisely $f_{\tau_{q, p}}(z)$ is not the focus of this thesis, therefore, some simple analysis is presented here to support the assumption that $\tau_{q, p}$ can be considered a uniformly distributed random variable for the MPRF case.

To that end, we can find the extreme values of engagement velocity and then simply assume a normal PDF between these values. The extreme negative relative velocity value will occur when the radar and target are flying directly at each other at maximum speed and the target is at its maximum roll rate. The extreme positive velocity will occur when the target is flying directly away from the radar at maximum speed while at maximum roll rate, and the radar is traveling at minimum speed. Table 4.1 shows the values used to determine the extreme velocities. They are all based on typical fighter aircraft performance and are applied to both radar and DRFM host platforms. Using the maximum roll rate and the wingspan results in a maximum of

Table 4.1: Typical Fighter Aircraft Specification Values

\begin{tabular}{|c|c|}
\hline Specification & Value \\
\hline \hline Maximum [Engagement] Speed & $1000 \mathrm{~km} / \mathrm{h}$ (Mach 0.8) \\
\hline Minimum Speed & $100 \mathrm{~km} / \mathrm{h}$ \\
\hline Maximum Roll Rate & $120^{\circ} / \mathrm{s}$ \\
\hline Wingspan & $13 \mathrm{~m}$ \\
\hline
\end{tabular}

$11 \mathrm{~m} / \mathrm{s}$ velocity due to aircraft maneuvering. The maximum possible closing velocity 
is twice the maximum speed, $2000 \mathrm{~km} / \mathrm{h}$ or $556 \mathrm{~m} / \mathrm{s}$. The maximum negative relative velocity will be: $-556 \mathrm{~m} / \mathrm{s}+-11 \mathrm{~m} / \mathrm{s}=-567 \mathrm{~m} / \mathrm{s}$. The maximum possible opening velocity is $1000 \mathrm{~km} / \mathrm{h}-100 \mathrm{~km} / \mathrm{h}=900 \mathrm{~km} / \mathrm{h}$ or $250 \mathrm{~m} / \mathrm{s}$. The maximum positive relative velocity will be: $250 \mathrm{~m} / \mathrm{s}+11 \mathrm{~m} / \mathrm{s}=261 \mathrm{~m} / \mathrm{s}$. To represent the PDF of the engagement velocity, $f_{v}(y)$, a normal PDF is used with $\mu_{v}=-153 \mathrm{~m} / \mathrm{s}$, corresponding to the midpoint of the range calculated and $\sigma_{v}=150 \mathrm{~m} / \mathrm{s}$.

$$
f_{v}(y)=\frac{1}{2 \pi \sigma_{v}^{2}} e^{\frac{\left(y-\mu_{v}\right)^{2}}{2 \sigma_{v}^{2}}}
$$

Figure 4.2(a) shows $f_{v}(y)$, while Figure 4.2(b) shows the amount the range can change due to the velocity during one PRI, $f_{\Delta R_{P R I}}(y)$. The transformation from (a) to (b)
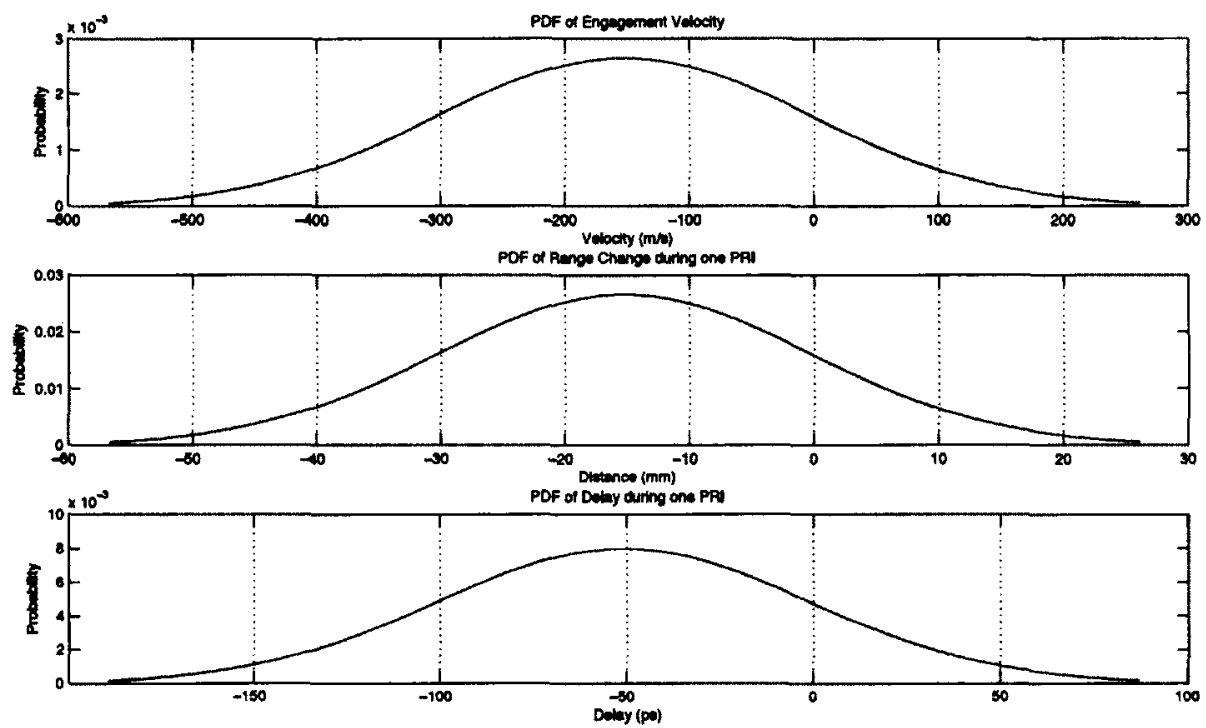

Figure 4.2: Development of $f_{V_{q}}(y)$. (a) PDF of Engagement Velocity, $f_{v}(y)$, (b) PDF of Range Change during one PRI $f_{\triangle R_{P R I}}(y)$, (c) $f_{V_{q}}(y)$

is:

$$
\Delta R_{P R I}=v \cdot T_{P R I}
$$

where $v$ is velocity and $T_{P R I}=100 \mu s$. In essence, this is a change in units from 
velocity to range. Therefore, the transformation is applied to both $\mu$ and $\sigma$. The result is $\mu_{\Delta R_{P R I}}=-15.3 m m, \sigma_{\triangle R_{P R I}}=15 \mathrm{~mm}$, and $f_{\triangle R_{P R I}}(y)$ is:

$$
f_{\Delta R_{P R I}}(y)=\frac{1}{2 \pi \sigma_{\Delta R_{P R I}}^{2}} e^{\frac{\left(y-\mu_{\Delta R_{P R I}}\right)^{2}}{2 \sigma_{\Delta R_{P R I}}^{2}}}
$$

Finally, Figure 4.2(c) shows $f_{V_{q}}(y)$, the delay that will be caused, negative or positive, during one PRI. The transformation from (b) to (c) is:

$$
V_{q}=\frac{\Delta R_{P R I}}{c}
$$

where $\mathrm{c}$ is the speed of light. Again, this is basically a change in units, from range to time and therefore $\mu_{V_{q}}=-51 p s, \sigma_{V_{q}}=50 p s$, and $f_{V_{q}}(y)$ is:

$$
f_{V_{q}}(y)=\frac{1}{2 \pi \sigma_{V_{q}}^{2}} e^{\frac{\left(y-\mu V_{q}\right)^{2}}{2 \sigma_{V_{q}}^{2}}}
$$

Therefore, from the relative velocities of the radar and target we have defined $V_{q}$, the random variable that contributes to delay based on velocity.

Second, let us analyse the $R_{q}$ term. To determine $f_{R_{q}}(x)$, we need to look at $\Delta R_{C P I}$, the change in range between CPI $q$ and CPI $q+1$. The same methodology for the derivation of $f_{V_{q}}(y)$ will be used. Figure 4.3(a) shows $f_{v}(y)$ again, from Figure (4.4). Figure 4.3(b) shows $f_{\triangle R_{C P I}}(x)$ which shows the amount that the range can change over the course of a CPI. It is derived from (a) with the following equation:

$$
\Delta R_{C P I}=v T_{C P I}
$$

where $T_{C P I}$, the duration of a CPI, is defined in (4.3). For the purposes of this thesis, it is assumed that $N_{C P I}=10$. Therefore, the result is $\mu_{\triangle R_{C P I}}=-153 \mathrm{~mm}$, 

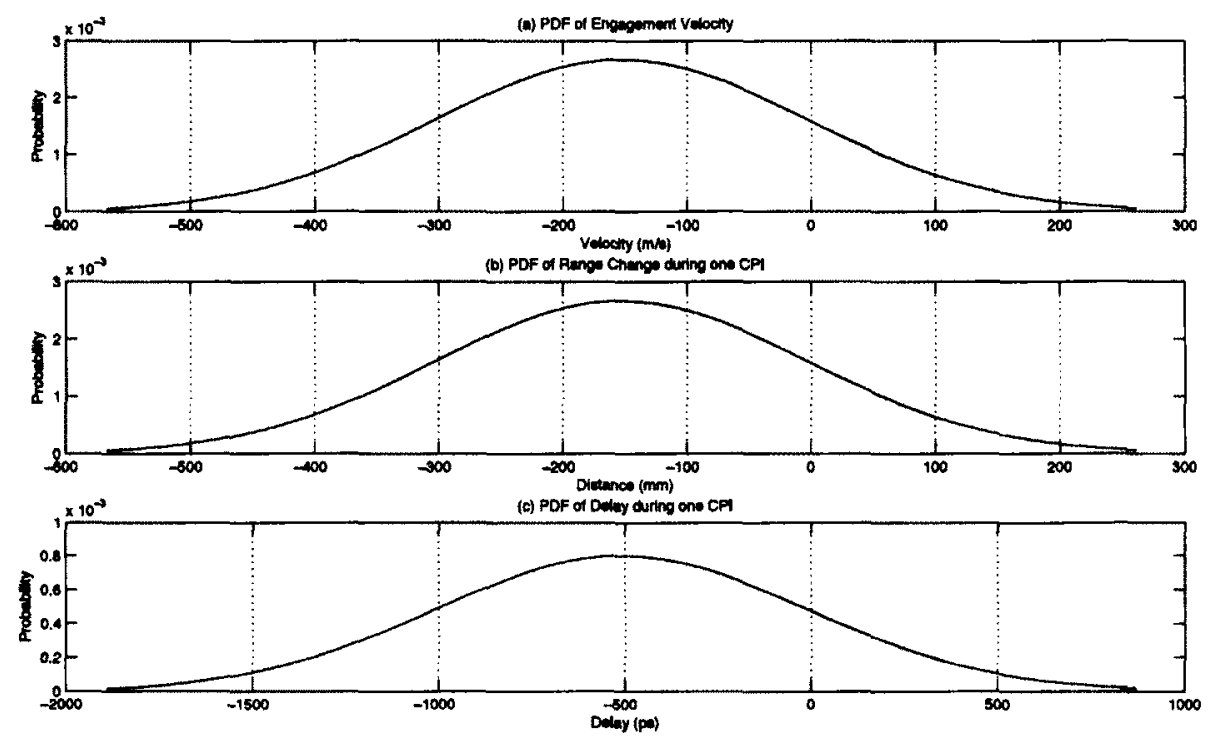

Figure 4.3: Development of $f_{R_{q}}(x)$. (a) PDF of Engagement Velocity, $f_{v}(x)$ (b) PDF of Range Change during one CPI $f_{\Delta R_{C P I}}(x),\left(\right.$ c) $f_{R_{q}}(x)$

$\sigma_{\triangle R_{C P I}}=150 \mathrm{~mm}$, and $f_{\triangle R_{C P I}}(x)$ is:

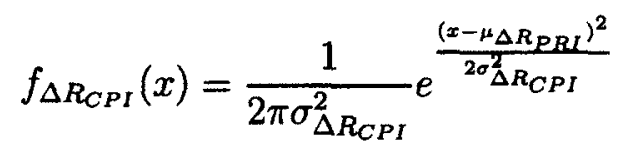

The overall delay that can occur during a CPI is shown in Figure 4.3(c) and was derived from (b) with the following equation:

$$
R_{q}=\frac{\Delta R_{C P I}}{c}
$$

Therefore, the result is $\mu_{R_{q}}=-510 p s, \sigma_{R_{q}}=500 p s$, and $f_{R_{q}}(x)$ is:

$$
f_{R_{q}}(x)=\frac{1}{2 \pi \sigma_{R_{q}}^{2}} e^{\frac{\left(x-\mu_{R_{q}}\right)^{2}}{2 \sigma_{R_{q}}^{2}}}
$$

From the relative velocities of the radar and target we have defined $R_{q}$, the random variable that establishes the amount of delay possible from CPI $q$ to CPI $q+1 . R_{q}$ 
is meant to establish the initial delay value for a CPI. However, as can be seen in the PDF, the value of $R_{q}$ could easily exceed the maximum delay value established in (4.1), $T_{s}$. This will be addressed next after we resolve the addition of the 2 PDFs.

Now that $R_{q}$ and $V_{q}$ have been defined and we have found their PDFs, we can now show that the $f_{\tau_{q, p}}(z)$ is a uniformly distributed random variable. From (2.13) we have the equation to determine the PDF resulting from the sum of two random variables, $Z=X+Y$. Applying (2.13) to (4.2) where $p=1$ results in the following:

$$
f_{\tau_{q, 1}}(z)=f_{R_{q}}(x) * f_{V_{q}}(y)
$$

Figure 4.4 shows (4.13) applied.
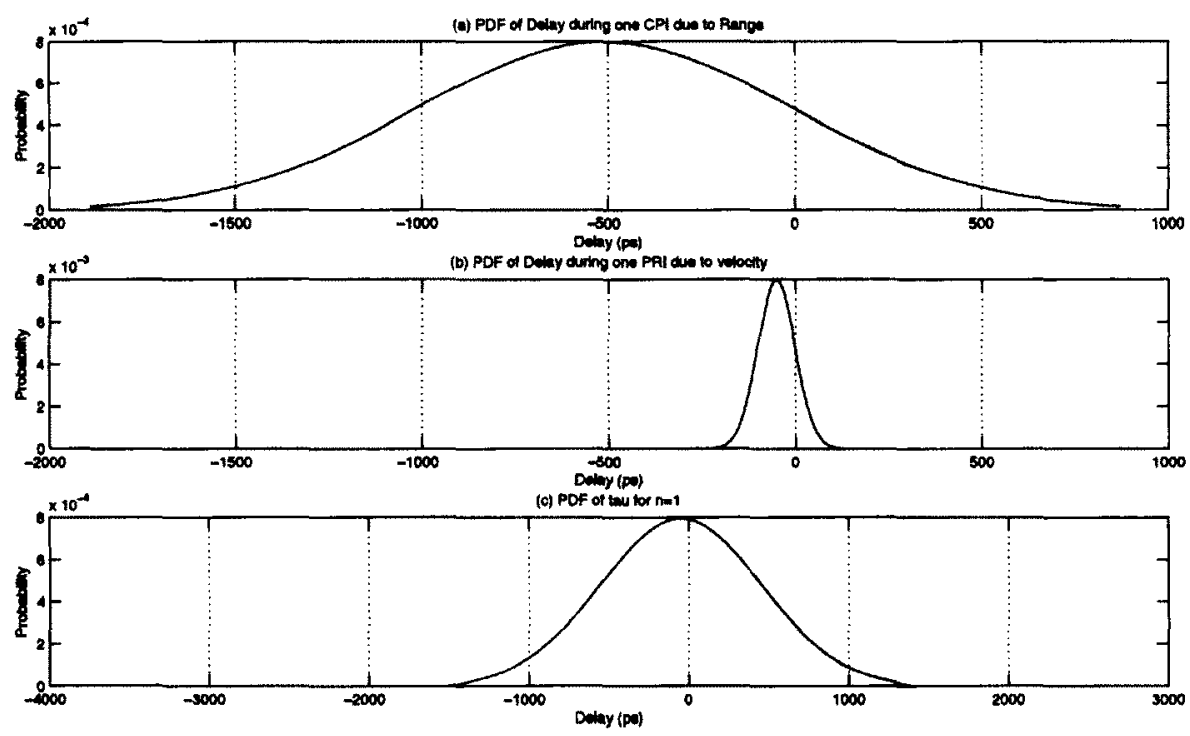

Figure 4.4: Development of $f_{\tau_{q, 1}}(z)$ (Unconstrained). (a) $f_{R_{q}}(x)$, (b) $f_{V_{q}}(y),($ c) $f_{\tau_{q, 1}}(z)$ (Unconstrained)

However, the overall delay is not very important. What is more important is where the delay will fall within one sample period. For example, given $T_{s}=150 \mathrm{ps}, \mathrm{a}$ $\tau$ of $1 p s, 151 p s$ and 301ps are all effectively the same, and the probabilities of those 

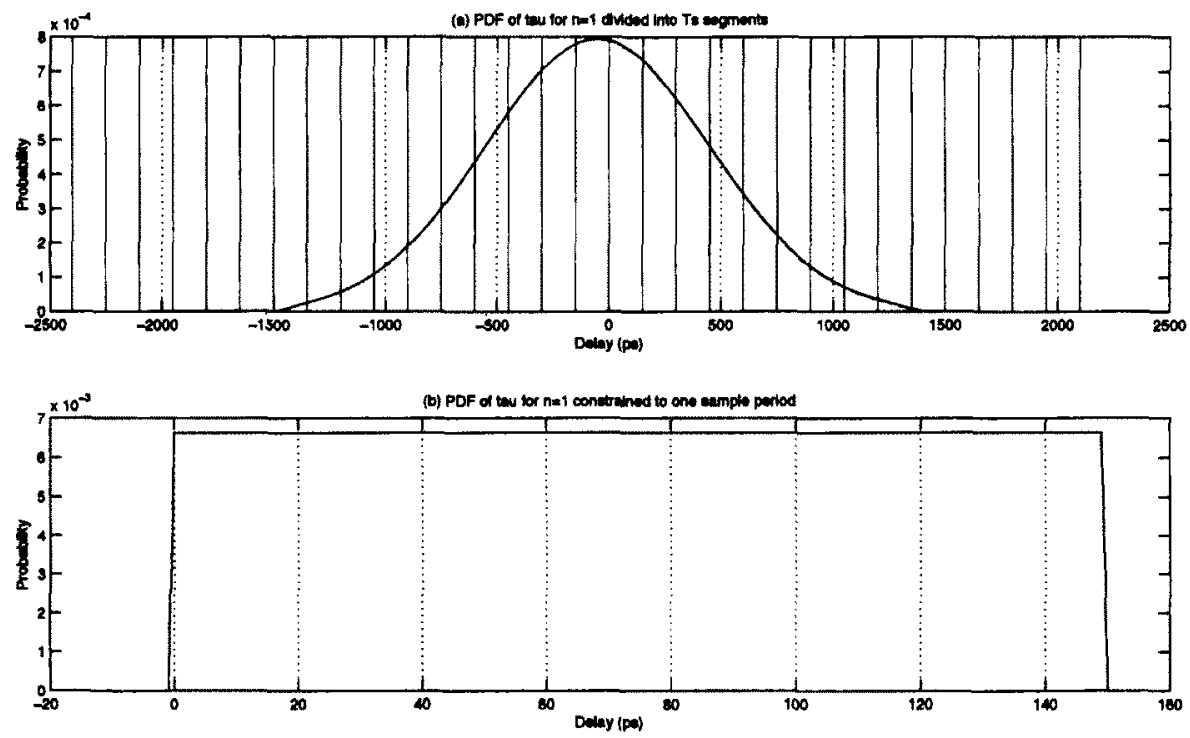

Figure 4.5: Development of $f_{\tau_{q, 1}}(z)$ (Constrained to one sample period). (a) $f_{\tau_{q, 1}}(z)$, divided into $T_{s}$ segments, (b) $f_{\tau_{q, 1}}(z)$ (Constrained to one sample period, $T_{s}$ )

delays are additive. Figure 4.5(a) shows $f_{\tau_{q, 1}}$ and divides it into segments $T_{s}$ wide. The additive probabilities of all the segments are shown in (b). The result is that $\tau_{q, 1}$ is a uniformly distributed random variable with a probability of approximately $\frac{1}{T_{s}}=\frac{1}{150}=6.667 \times 10^{-3}$ as expected.

However, it needs to be proven that the more general $\tau_{q, p}$ can be simulated by a uniformly distributed random variable for all values of $q$ and $p$. Therefore, we must apply (2.13) to (4.2) for all values of $p$. For $p=2$ the result is as follows:

$$
f_{\tau_{q, 2}}(z)=\left[f_{R_{q}}(x) * f_{V_{q}}(y)\right] * f_{V_{q}}(y)
$$

As can be seen, for each increase in $p$, a convolution with $f_{V_{q}}(y)$ is added. The simulation in this thesis uses a CPI of $10\left(N_{C P I}=10\right)$ which means that $p$ takes the values of 0 to 9 during each CPI. Figures 4.6 and 4.7 , show $f_{\tau_{q, 5}}(z)$ and $f_{\tau_{q, 9}}(z)$ respectively. Both are uniformly distributed with a probability of approximately $\frac{1}{T_{s}}$ 

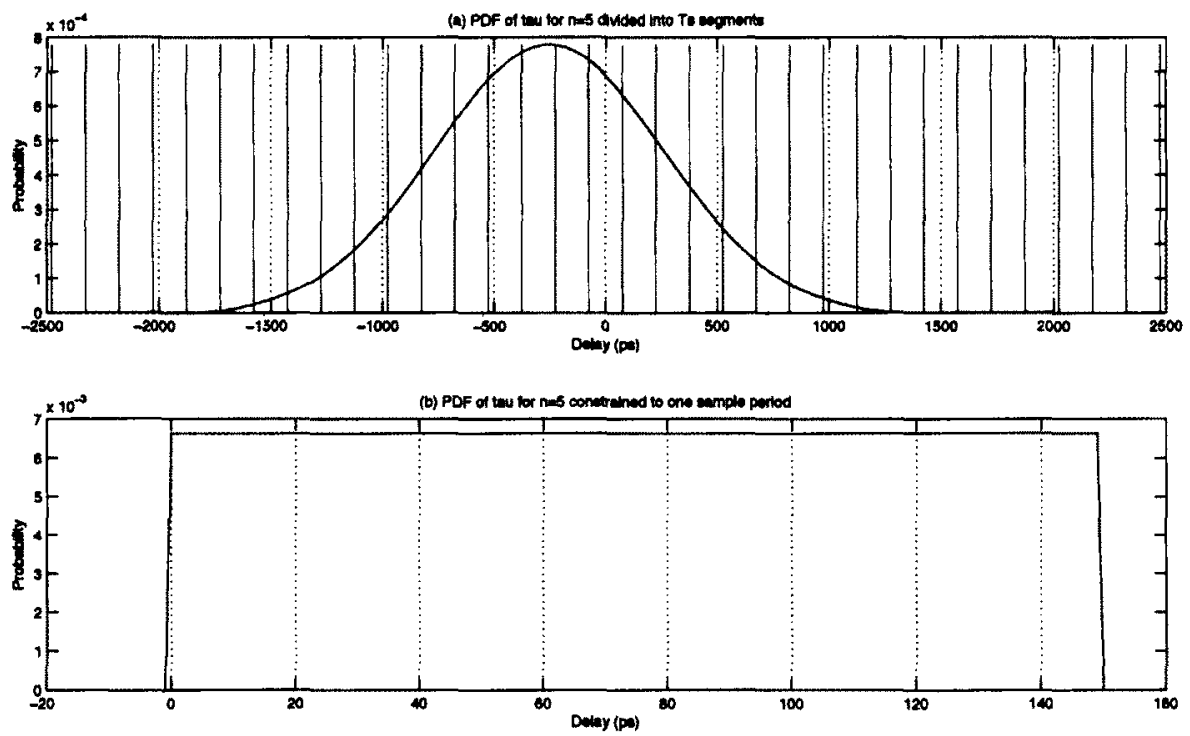

Figure 4.6: Development of $f_{\tau_{q, 5}}(z)$ (Constrained to one sample period. (a) $f_{\tau_{q, 5}}(z)$, divided into $T_{s}$ segments, (b) $f_{\tau_{q, 5}}(z)$ (Constrained to one sample period, $T_{s}$ )

as was $f_{\tau_{q, 1}}(z)$.

It has therefore been proven that, for the MPRF case, $\tau_{q, p}$ can simply be modeled as a uniformly distributed random variable with a probability of $\frac{1}{T_{s}}$. This means that regardless of the value of $q$ and $p, \tau$ will have the same PDF. Therefore, the EP technique and simulation will use the following definition of $f_{\tau}(z)$ :

$$
f_{\tau}(z)=\frac{1}{T_{s}}, 0<z<T_{s}
$$

In other words, the simulation will select a new random value for the sampling delay after each pulse, based on the PDF given in (4.15).

Earlier in the explanation of this phenomenon we took the perspective of a receiver, either the radar or DRFM receiver. We now know the PDF for the delay at these receivers, $f_{\tau}(z)$. However, what has not been analysed is the full path of a signal through a DRFM jammer and back to the radar. In fact, for this signal path, the 

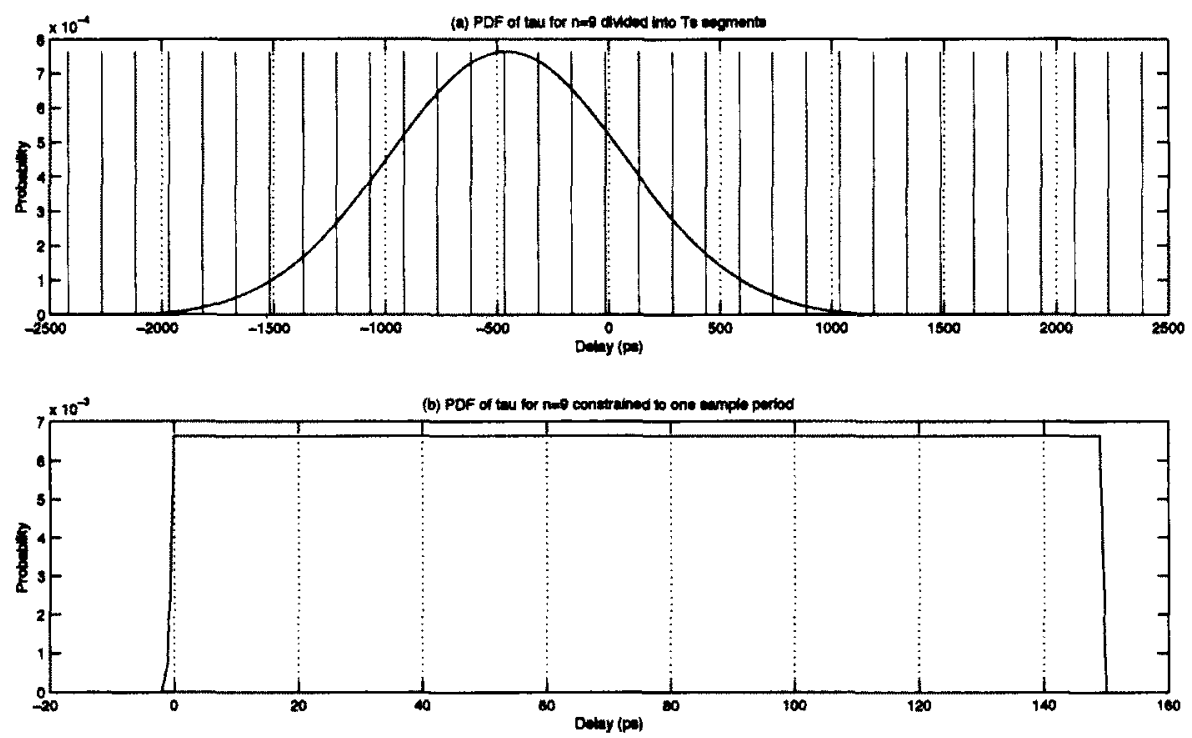

Figure 4.7: Development of $f_{\tau_{q, 9}}(z)$ (Constrained to one sample period). (a) $f_{\tau_{q, 9}}(z)$, divided into $T_{s}$ segments, (b) $f_{\tau_{q, 9}}(z)$ (Constrained to one sample period, $T_{s}$ )

sample delay phenomenon will occur twice-once at the DRFM receiver and once at the radar receiver. This opens an opportunity for the radar since the DRFMs return will be slightly more distorted than the skin return.

In summary, the dynamic nature of electronic warfare engagements coupled with the fact that a DRFM jammer must digitize received signals, gives a radar an opportunity to perform EP in order to distinguish between a target return and a DRFM return. The next section will present the $\mathrm{CRN}$ technique and show how it uses (4.15) in the radar EP processor to seize this opportunity.

\subsubsection{EP Processor}

In order to explain how to exploit this incoherent sampling phenomenon, the concept of an EP Processor is now introduced. The EP processor is envisioned as a module of the radar that generates and processes $\mathrm{CRN}$ pulses. The radar transmitter receives a short CRN pulse from the EP processor, on the order of $10 \%$ of the length of 
the regular radar pulse. This CRN pulse is then concatenated to the radar pulse outside the EP processor and then the combined pulses are transmitted. The radar receiver will receive target returns, which could possibly be DRFM returns, do any pre-processing required such as AGC, decatenate the CRN pulse from the radar pulse and then pass the CRN pulse to the EP processor. The EP processor will then process the received CRN pulse, perform integration and then report to the radar tracker whether that certain target is in fact a target or is actually a DRFM jammer return. The next section will provide details on how the design of the EP Processor enables this.

\subsection{The CRN Technique, Design and Simulation}

This section will present the CRN technique, proposed EP processor high-level design, and the MATLAB simulation used to demonstrate both, in full detail. It will be organized into the main modules of the design: EP Waveform Generator, EP Receiver and DRFM. For each module, its technique, design and simulation will be presented together with the aid of flow charts. Finally, a section will explain how simulation results for the complete CRN technique are compiled, calculated and presented.

\subsubsection{EP Waveform Generator}

The EP waveform generator and its integration with a generic radar is shown in Figure 4.8. The EP Waveform Generator simulation only simulates in the digital domain and it assumes that there would be Direct Digital Conversion (DDC). The EP waveform generator has two components and one data item. The two components are the Noise Generator and Low Pass Filter (LPF). The data item is the Matched Filter Coefficients. Figure 4.8 also shows the radar components involved in the EP technique. They are the Concatenator, Modulator, and Band Pass Filter (BPF). All 


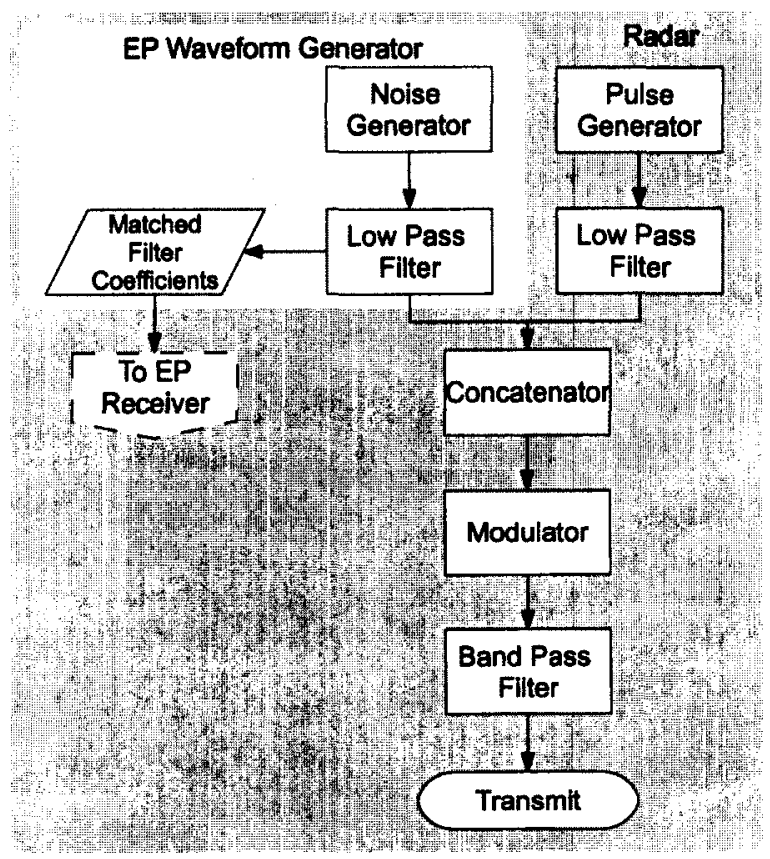

Figure 4.8: Flowchart for the EP Waveform Generator

will now be described in detail.

\section{Noise Generator}

The noise generator is a simple component that generates white Gaussian noise. It is a key component for the CRN technique that relies on that fact that a DRFM will distort random noise, especially at frequencies approaching $\frac{f_{3}}{2}$. In terms of the simulation, the noise generator uses the MATLAB wgn() function. The noise signal will be referred to as $x_{c r n}(n)$ and it will have a length of $N_{c r n}$ samples. 


\section{Low Pass Filter}

Before the CRN pulse is passed to the radar to be concatenated to the radar pulse it is passed through a LPF. This is simply to make sure that $x_{c r n}$ is at the proper bandwidth before it is passed to the radar. In the simulation, an equiripple FIR filter was used with a cut-off frequency equal to the bandwidth, $B$. Three configurations were simulated, with $B=0.333, B=0.2$, and $B=0.15$ in normalized frequency, respectively. Given the sampling frequency assumed earlier $f_{s}=6.667 G \mathrm{~Hz}$, those normalized bandwidths equate to $1.11 \mathrm{GHz}, 667 \mathrm{MHz}$ and $500.25 \mathrm{MHz}$ respectively. The the matched filter coefficients that will be passed to the EP Receiver are gleaned from the output of the low pass filter.

\section{Matched Filter Coefficients}

From (2.10) we know that the coefficients are derived from the complex conjugate of the signal to be matched, reversed in time. In this case, $x_{c r n}(n)$ is not complex, so we simply need to reverse it in time. Therefore, the matched filter coefficients, $b_{l}$, will be

$$
b_{l}=x_{c r n}\left(N_{c r n}-l\right), 0<l<N_{c r n}-1
$$

where $l$ is the index for $b_{l}$.

\section{Concatenator}

The concatenator component in Figure 4.8 was not included in the simulation, since it is only meant to simulate the CRN pulse. However, it is shown as a place holder. In a simulation involving the entire radar, the radar pulse and CRN pulse would be concatenated in this component before being sent to the modulator as one contiguous pulse. It would be the desirable to have the CRN pulse after the radar pulse so as not to affect any leading edge detection that the radar is designed to do. 


\section{Modulator}

The modulator component was implemented using the MATLAB function modulate() using frequency modulation with a center frequency of $\omega_{c}$ and a bandwidth $B$.

\section{Band Pass Filter}

The BPF was implemented with an equiripple FIR filter with a center frequency $\omega_{c}$ and a bandwidth of $B$. This is the last component to be simulated on the transmit side of the radar.

In a physical implementation, the signal would need to be put through a DAC in order for it to be transmitted. However, since it is assumed that the radar and DRFM have equivalent digitization capabilities, this component is omitted and all of the simulations are done in the digital domain. A consequence of this is the effects of quantization noise are not simulated. As stated in Section 6.2.3, this is an opportunity for future research and to further explore the feasibility of the CRN technique.

\subsubsection{EP Receiver}

The EP Receiver and its integration with a generic radar is shown in Figure 4.9. The EP Receiver has five components and two data item. The six components are the Low Pass Filter, Matched Filter, Magnitude Detector, Square Law Integrator, Threshold Detector, and $\mathrm{m}$ of $\mathrm{n}$ Integrator. The data items are the Matched Filter Coefficients (explained in 4.2.1) and the final Decision-whether the target under evaluation is a target or a DRFM. The components of the radar that are also involved in the CRN technique and displayed in Figure 4.9 are: Band Pass Filter, Demodulator and Decatenator. Finally, some components are shown that are required for simulation purposes only. These are the Noise and Random Delay Simulations. All of the 


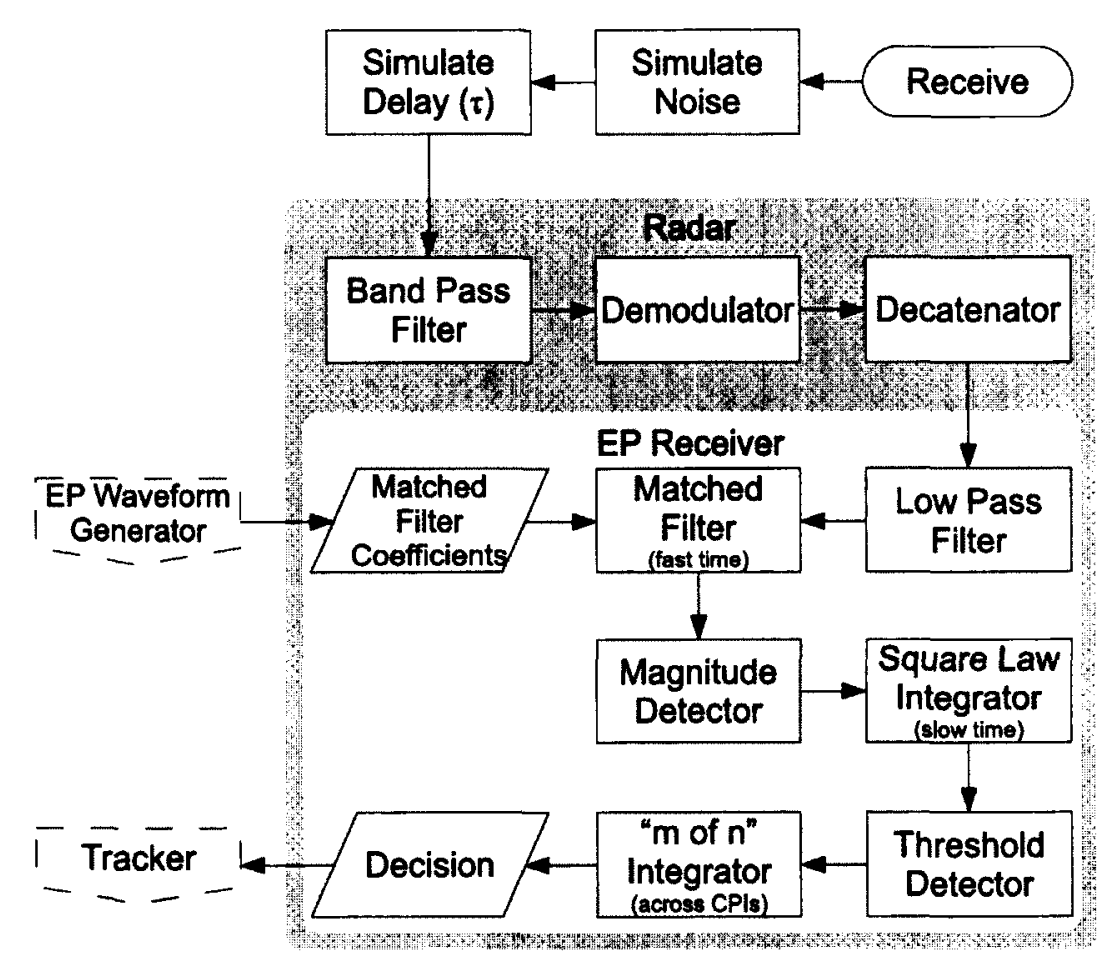

Figure 4.9: Flowchart for the EP Receiver

components in Figure 4.9 will now be described in detail and in order of the flow chart.

\section{Simulate Noise}

The Simulate Noise component of the EP receiver uses the MATLAB awgn() function to provide additive white Gaussian noise to the received CRN pulse to simulate the added noise from the transmitter, receiver and environment, and the attenuation of the signal. This noise is denoted as $x_{n}$ in (4.18). This component is the only component of the EP receiver simulation that knows, for simulation purposes, if the received CRN pulse is a target skin return or a DRFM return. It requires this information to allow the simulation of a slightly higher SNR for the DRFM compared to the skin, as would be the case in a real engagement. This component uses the 
parameters $S N R_{\text {radar }_{s k i n}}, S N R_{\text {radar }_{d r f m}}$ and $\triangle S N R$ to determine the amount of noise added to the received CRN pulse. The relationship between these parameters is:

$$
S N R_{\text {radar }_{d r f m}}=S N R_{\text {radar }_{s k i n}}+\Delta S N R
$$

\section{Simulate Random Delay}

The simulate random delay component simulates incoherent sampling by implementing the phenomenon discussed earlier and specifically implements $(4.15), f_{\tau}(z)$. This component generates a random delay value based on $f_{\tau}(z)$ and then uses that delay value to interpolate between all samples in the received $x_{c r n}(n)$ signal. The result will be the delayed signal, $x_{c r n+\tau}^{\prime}(n)$ which is defined as

$$
\begin{aligned}
x_{c r n+\tau}^{\prime}(n) & =x_{c r n}(n)-x_{\tau}^{\prime}(n)+x_{n}(n) \\
x_{\tau}^{\prime}(n) & =\frac{\tau}{T_{s}} \cdot\left(x_{c r n}(n)-x_{c r n}(n-1)\right)
\end{aligned}
$$

where $x_{n}(n)$ is white gaussian noise. Equation (4.19) describes the interpolation of $x_{c r n}$. Essentially, the second term forms a line between two sample points, $x_{c r n}(n)$ and $x_{c r n}(n-1)$, and the first term chooses which point along the line to use as the new sampling point based on the sampling delay, $\tau$. In the end, $x_{\tau}(n)$ represents the value that needs to be subtracted from $x_{c r n}(n)$ so that $x_{c r n+\tau}^{\prime}(n)$ has the correct re-sampled value. For clarity in the explanations to come, we wish to abstract this interpolation process out and leave only the magnitude difference that results from the interpolation. Therefore, we move the term $\frac{\tau}{T_{s}}$ from (4.19) to (4.18) to result in this definition of $x_{c r n+\tau}(n)$ and $x_{\tau}(n)$ :

$$
\begin{aligned}
x_{c r n+\tau}(n) & =x_{c r n}(n)-\frac{\tau}{T_{s}} \cdot x_{\tau}(n)+x_{n}(n) \\
x_{\tau}(n) & =x_{c r n}(n)-x_{c r n}(n-1)
\end{aligned}
$$




\section{Band Pass Filter}

The BPF was implemented with an equiripple FIR filter with a center frequency $\omega_{c}$ and a bandwidth of $B$. This filter is used to stop aliasing.

\section{Demodulator}

The demodulator component was implemented using the MATLAB function demod() using frequency modulation with a center frequency of $\omega_{c}$ and a bandwidth $B$.

\section{Decatenator}

The decatenator component in Figure 4.9 is the reciprocal component to the concatenator of the EP Waveform Generator. The decatenator was also not included in the simulation. However, it is shown as a place holder since this simulation is only meant to simulate the CRN pulse. In a simulation involving the entire radar, the CRN pulse would be decatenated or separated from the radar pulse in this component before being sent to the EP Receiver.

\section{Low Pass Filter}

In the simulation, an equiripple finite impulse response (FIR) filter with a cut-off frequency equal to the bandwidth $B$ was used to implement the LPF in the EP Receiver. Its main purpose is anti-aliasing.

\section{Matched Filter}

The matched filter is where processing for the CRN technique truly begins. The matched filter coefficients are passed from the noise generator of the EP waveform generator to the EP receiver after each CRN pulse is created. In other words the matched filter is working on fast time data. The matched filter is created with an 
FIR filter with coefficients, $b_{l}$, defined in (4.16). The received, delayed, filtered and demodulated CRN pulse, which will be denoted $x_{p}$, received from the LPF, is passed through the matched filter using the MATLAB function filter().

At this point we can classify the input to the matched filter, $x_{p}$, into two different forms, defined as

$$
\begin{aligned}
x_{p_{s k i n}}(n) & =x_{c r n+\tau}(n) \\
& =x_{c r n}(n)-\frac{\tau_{r}}{T_{s}} \cdot x_{\tau_{r}}(n)+x_{n}(n) \\
x_{p_{d r f m}}(n) & =x_{c r n+2 \tau}(n) \\
& =x_{c r n}(n)-\frac{\tau_{r}}{T_{s}} \cdot x_{\tau_{r}}(n)-\frac{\tau_{d}}{T_{s}} \cdot x_{\tau_{d}}(n)+x_{n}(n)
\end{aligned}
$$

where $x_{p_{s k i n}}(n)$ represents a return from the skin of the target and $x_{p_{d r f m}}(n)$ represents a return from the DRFM jammer. For the skin return we define $x_{c r n+\tau}(n)$ as the generated noise from the EP waveform generator $x_{c r n}(n)$, with the random delay effects at the radar receiver $\frac{\tau_{r}}{T_{0}} \cdot x_{\tau_{r}}(n)$ and noise $x_{n}(n)$. The DRFM return is defined almost the same, save the added random delay effects at the DRFM receiver, $\frac{\tau_{d}}{T_{s}} \cdot$ $x_{\tau_{d}}(n)$. It should be noted that $\tau_{r}$ and $\tau_{d}$ are both random variables and the values of $\tau_{r}$ in (4.22) and (4.23) are not equal, since the DRFM cannot transmit its replica at the same instant the CRN pulse reflects from the skin. They will be different random numbers.

Included here is a simple analysis of (4.22) and (4.23) and the resultant outputs from the matched filter. In fact, all signals denoted by an $x$ are random noise, therefore an extensive amount of stochastic mathematics need to be derived. For the purposes of this Masters thesis, a simulation in MATLAB is used to prove the CRN concept, vice the stochastic theory.

Using (2.6) we can define the difference equation for the matched filter applied to 
the generic $x_{p}$ as

$$
\begin{aligned}
y(n) & =b_{0} x_{p}(n)+b_{1} x_{p}(n-1)+\ldots+b_{N_{c r n}-1} x_{p}\left(n-N_{c r n}+1\right) \\
& =\sum_{k=0}^{N_{c r n}-1} b_{l} x_{p}(n-k)
\end{aligned}
$$

where $y(n)$ is the generic output of the matched filter. Figure 4.10 shows a typical output from the matched filter, $y(n)$. The large spike of $y(n)$ in Figure 4.10 occurs

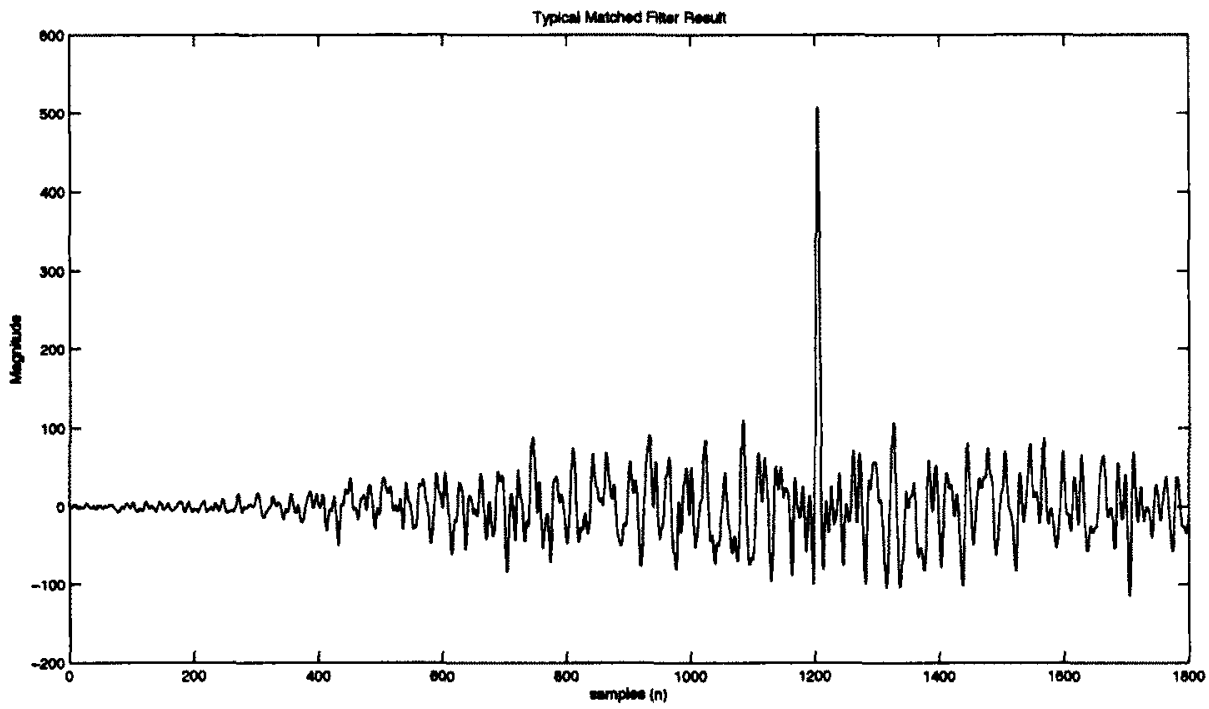

Figure 4.10: Typical Matched Filter Result

when each sample of $x_{c r n}$ component of $x_{p}$ aligns with the proper matched filter coefficient in $b_{l}$. The magnitude of the spike is dependent on the $x_{n}(n)$ and $x_{\tau}(n)$ components of $x_{p}(n)$. We define $y_{s k i n}(n)$ and $y_{d r f m}(n)$ as the matched filter outputs corresponding to $x_{p_{s k i n}}$ and $x_{p_{d r f m}}$ respectively.

$$
\begin{aligned}
y_{s k i n}(n) & =y_{c r n}(n)-\frac{\tau_{r}}{T_{s}} \cdot y_{\tau_{r}}(n)+y_{n}(n) \\
-\quad y_{d r f m}(n) & =y_{c r n}(n)-\frac{\tau_{r}}{T_{s}} \cdot y_{\tau_{r}}(n)-\frac{\tau_{d}}{T_{s}} \cdot y_{\tau_{d}}(n)+y_{n}(n)
\end{aligned}
$$




$$
y_{s k i n}(n)-y_{d r f m}(n)=\frac{\tau_{d}}{T_{s}} \cdot y_{\tau_{d}}(n)
$$

In these equations $y_{c r n}, y_{\tau_{r}}, y_{\tau_{d}}$, and $y_{n}$ are simply the matched filter results of the $x_{p}$ components. Equation (4.27) is the subtraction of (4.25) and (4.26). This is a simplified operation on these equations that assumes that the expected values for common terms are equal in (4.25) and (4.26). It serves to illustrate the crux of the CRN technique-after matched filtering the skin return will have a slightly higher expected value than that of the DRFM return. That is:

$$
E\left(\max \left(y_{d r f m}(n)\right)\right)<E\left(\max \left(y_{s k i n}(n)\right)\right)
$$

The output from the matched filter will be the generic $y(n)$. The EP Receiver does not yet know whether $y(n)$ is defined by (4.25) or (4.26). The following components will determine this.

\section{Magnitude Detector}

The magnitude detector receives the output from the matched filter $y(n)$ and finds the maximum magnitude of the signal, $y_{\max }$, where $y_{\max }$ is classified as one of:

$$
\begin{aligned}
y_{\text {max }} x_{k i n} & =\max \left(y_{c r n}(n)-\frac{\tau_{r}}{T_{s}} \cdot y_{\tau_{r}}(n)+y_{n}(n)\right) \\
y_{\text {max }_{d r f m}} & =\max \left(y_{c r n}(n)-\frac{\tau_{r}}{T_{s}} \cdot y_{\tau_{r}}(n)-\frac{\tau_{d}}{T_{s}} \cdot y_{\tau_{d}}(n)+y_{n}(n)\right)
\end{aligned}
$$

The output of the magnitude detector is simply $y_{\max }$, as we have yet to determine if the received signal can be defined by (4.29) or (4.30). 


\section{Square Law Integrator}

The square law integrator integrates all the values of $y_{\max }[p]$, where $p$ represents the pulse within a CPI of $N_{C P I}$ pulses. In common radar terms, the square law integrator is operating in slow time. Using (2.12) we can determine the output of the square law integrator to be:

$$
|y|^{2}=\sum_{p=0}^{N_{C P I}-1}\left|y_{\max }[p]\right|^{2}
$$

This step basically grows the difference between the value of $|y|^{2}$ if it is defined by $\left|y_{s k i n}\right|^{2}$ or $\left|y_{d r f m}\right|^{2}$ which are defined in (4.32) and (4.33) where $i_{p}$ is the index of the maximum value of $y(n)$ found by the magnitude detector for pulse $p$ :

$$
\begin{aligned}
\left|y_{s k i n}\right|^{2} & =\sum_{p=0}^{N_{C P I}-1}\left|y_{c r n}\left(p, i_{p}\right)-\frac{\tau_{r}}{T_{s}} \cdot y_{\tau_{r}}\left(p, i_{p}\right)+y_{n}\left(p, i_{p}\right)\right|^{2} \\
\left|y_{d r f m}\right|^{2} & =\sum_{p=0}^{N_{C P I}-1}\left|y_{c r n}\left(p, i_{p}\right)-\frac{\tau_{r}}{T_{s}} \cdot y_{\tau_{r}}\left(p, i_{p}\right)-\frac{\tau_{d}}{T_{s}} \cdot y_{\tau_{d}}\left(p, i_{p}\right)+y_{n}\left(p, i_{p}\right)\right|^{2}(4
\end{aligned}
$$

Following the same logic as Equation (4.27) we can subtract (4.33) from (4.32) to show how the difference in the two possible forms of $|y|^{2}$ has been grown:

$$
\left|y_{s k i n}\right|^{2}-\left|y_{d r f m}\right|^{2}=\sum_{p=0}^{N_{C P I-1}}\left|\frac{\tau_{d}}{T_{s}} \cdot y_{\tau_{d}}\left(p, i_{p}\right)\right|^{2}
$$

In essence, the difference has been been squared and multiplied by $N_{C P I}$. This is a crucial step that allows a threshold value to be selected to give more consistent detection of the presence of the $\frac{\tau_{d}}{T_{s}} \cdot y_{\tau_{d}}(n)$ distortion.

\section{Threshold Detector}

The threshold detector is a simple component that makes the first attempt at a decision. The decision will be between two hypotheses: 
1. The measurement, $|y|^{2}$, is a result of a target skin return $\left(H_{0}\right)$

2. The measurement, $|y|^{2}$, is a result of a DRFM jamming signal $\left(H_{1}\right)$

These hypotheses are denoted $H_{0}$ and $H_{1}$ respectively. Here an assumption is made that the radar will not pass a signal to the EP Processor that is a result of noise, interference or clutter. This assumption is made to control the scope of this thesis, but thoughts on how to cope with this possibility are included in Section 6.2.2. A threshold $T$ is used to choose between $H_{0}$ and $H_{1}$ in the following fashion:

$$
|y|^{2} \underset{H_{0}}{\stackrel{H_{1}}{>}} T
$$

The value of $\mathrm{T}$ is found through experimentation for each simulation scenario, and it is static at run-time. An adaptable threshold algorithm is left as an open area of research, see Section 6.2.1. The output of the threshold detector will be a decision based on $T$, that will be denoted by $D_{T}$. It's value can be either $H_{0}=0$ or $H_{1}=1$.

\section{m of $\mathbf{n}$ Detector}

As described in Section 2.4.2, an $m$ of $n$ detector simply takes $n$ binary inputs and applies a threshold of $m$ to output a binary decision. In this application, the $m$ of $n$ detector receives $n$ values of $D_{T}$, each representing the decision made from one CPI. If $m$ or more values of $H_{1}$ are received the final decision will be $D=H_{1}$. If fewer than $m$ are received the final decision will be $D=H_{0}$. The equation implemented is:

$$
\sum_{i=0}^{n-1} D_{T}(i) \underset{H_{0}}{\stackrel{H_{1}}{>}} m
$$

In the simulation, the values used are $m=3$ and $n=5$. 


\section{Decision}

The final decision $D$ as described above indicates whether the target being processed is a skin return or DRFM return. This information would be passed to the radar tracker indicating whether it should drop a jammer being tracked or continue tracking a true target.

\subsubsection{DRFM}

The DRFM jammer is simulated quite simply since the CRN technique does not rely on any of the processing done by the DRFM. It solely relies on the DRFM incoherently sampling the CRN pulse. Figure 4.11 shows the flowchart of the DRFM as simulated for this thesis. The DRFM has been abstracted to only three components, two BPFs

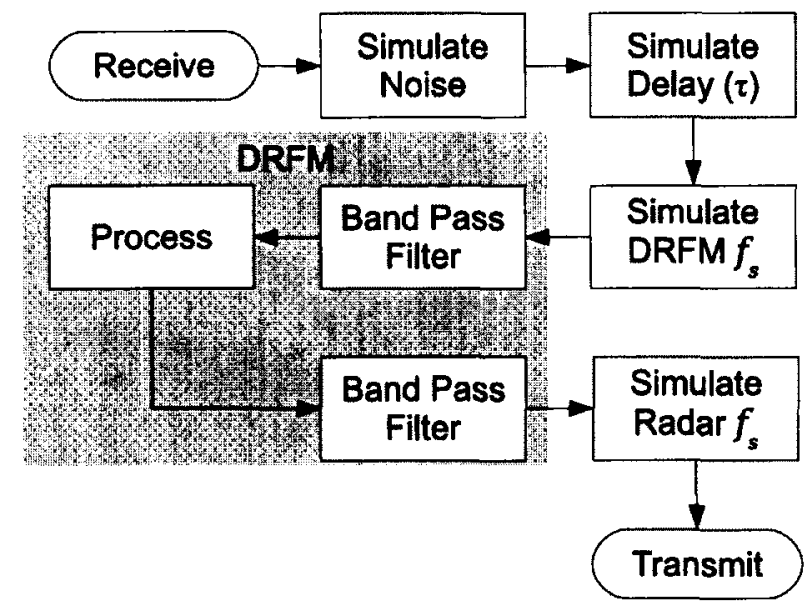

Figure 4.11: Flowchart for the DRFM Jammer

and a Processor. In reality there would be many more physical components that compose a DRFM jammer. The two BPFs are exact replicas of the radar receiver's $\mathrm{BPF}$, and the processor does nothing but pass the signal through. Figure 4.11 also shows four simulation components that would not be physical components of a DRFM 
but are included in the simulation. They are Simulate Noise, Simulate Random Delay $(\tau)$, Simulate DRFM $f_{s}$ and Simulate Radar $f_{s}$. They will each be discussed below.

\section{Simulate Noise}

The Simulate Noise component of the DRFM uses the MATLAB awgn() function to provide additive white Gaussian noise to the received CRN pulse to simulate the added noise from the transmitter, receiver and environment, and the attenuation of the signal. The parameter used was $S N R_{d r f m}=40 d B$.

\section{Simulate Random Delay $(\tau)$}

The simulate random delay uses the same algorithm as described in Section 4.2.2 to simulate the fact that the DRFM is incoherently sampling the CRN pulse.

\section{Simulate DRFM $f_{s}$}

This simulation component simply includes the fact that the DRFM likely has a different sampling frequency, $f_{s_{d}}$, than the radar, $f_{s_{r}}$. It uses the MATLAB function resample () to convert from $f_{s_{r}}$ to $f_{s_{d}}$. The simulation uses the values:

$$
\frac{f_{s_{d}}}{f_{s_{r}}}=\frac{11}{10}
$$

in normalized frequency.

\section{Simulate Radar $f_{s}$}

The simulate radar $f_{s}$ component is simply the complement to the simulate DRFM $f_{s}$ component. It converts from $f_{s_{d}}$ to $f_{s_{r}}$ using the ratio in (4.37). 


\subsubsection{Simulation Results}

This section will explain how the simulation is run and results are calculated. The actual results will be presented in Chapter 5 . Figure 4.12 shows, at an abstracted level, the flowchart of the CRN technique simulation. It is broken down into two simulation components: the Trial Control and Simulation Control, which will be discussed individually below.

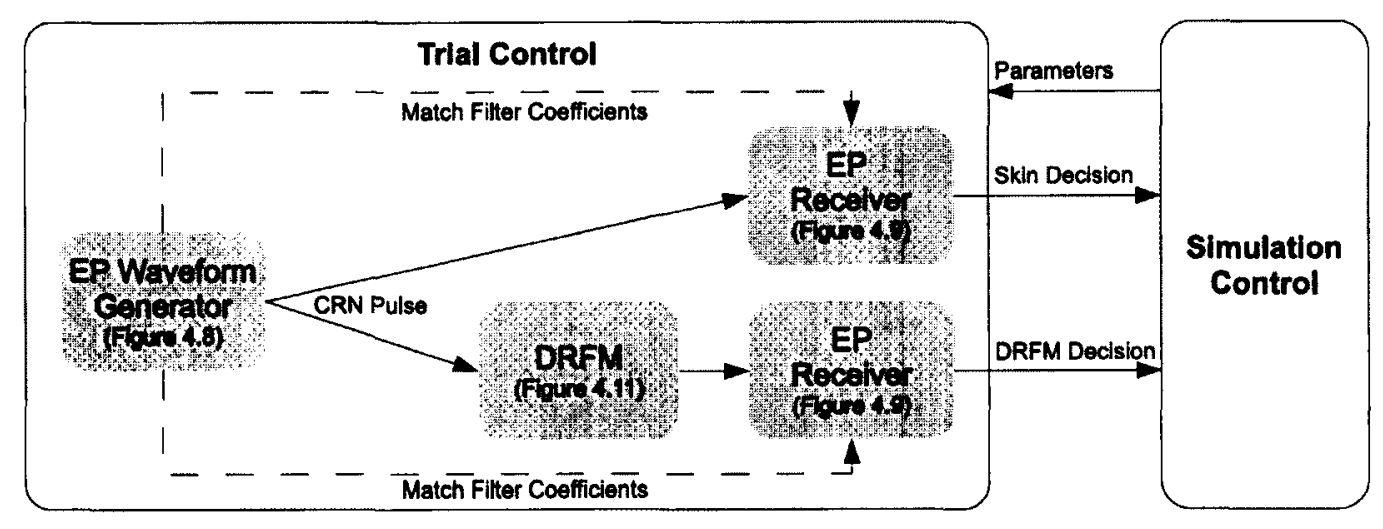

Figure 4.12: Flowchart of the CRN Technique Simulation

\section{Trial Control}

This component contains the three main modules of the CRN technique as described in the previous sections. The trial control component controls these three modules over the course of $n$ CPIs. The trial control first receives the parameters to be simulated for this trial. Those parameters' typical values and descriptions are shown in Table 4.2. Using these parameters the three main modules will operate as explained in the previous sections. The trial control simply controls the passing of parameters, data items and signals between modules. As shown in Figure 4.12 the EP Waveform Generator creates CRN pulses and the same pulses are passed through the skin signal path and DRFM signal path to the EP receiver. This allows for direct comparison of 
Table 4.2: Parameter Descriptions for the CRN Technique Simulation

\begin{tabular}{|c|c|l|}
\hline Parameter & Typical Value(s) & Description \\
\hline \hline$k$ & 25,50 & number of trials to perform \\
\hline$m$ & 3 & for the $m$ of $n$ detector \\
\hline$n$ & 5 & for the $m$ of $n$ detector \\
\hline$N_{C P I}$ & 10 & Number of pulses in a CPI \\
\hline$T_{P R I}$ & $100 \mu s$ & Duration of a PRI \\
\hline$T$ & $10^{4}-10^{6}$ & Threshold for the Threshold Detector \\
\hline$N_{\text {crn }}$ & $600,1200,2400$ & Length of CRN pulse in samples \\
\hline$\omega_{c}$ & $0.8,0.86,0.89$ & $\begin{array}{l}\text { Center frequency of all BPFs and modula- } \\
\text { tors, in normalized frequency }\end{array}$ \\
\hline$B$ & $0.333,0.2,0.15$ & $\begin{array}{l}\text { Bandwidth of all filters, in normalized fre- } \\
\text { quency }\end{array}$ \\
\hline$S N R_{\text {radar }_{s i n}}$ & $-3-30 \mathrm{~dB}$ & SNR at the radar receiver for a skin return \\
\hline$\Delta S N R$ & $1,2,3 \mathrm{~dB}$ & $\begin{array}{l}\text { difference in SNR between the DRFM and } \\
\text { skin return at the radar receiver }\end{array}$ \\
\hline$S N R_{d r f m}$ & $40 \mathrm{~dB}$ & SNR at the DRFM receiver \\
\hline$\frac{f_{s_{d}}}{f_{s_{r}}}$ & $\frac{11}{10}$ & $\begin{array}{l}\text { Ratio of DRFM sampling frequency to radar } \\
\text { sampling frequency }\end{array}$ \\
\hline
\end{tabular}

the two paths. The two EP receivers shown are actually implemented with the same MATLAB function which is executed twice. This ensures that the skin return and DRFM return are processed with the exact same EP receiver. The resultant decisions for both the skin signal path, $D_{s k i n}(k)$, and DRFM signal path, $D_{d r f m}(k)$, are passed to the Simulation Control.

\section{Simulation Control}

This simulation component can run simulations which vary one parameter with $j$ different values. For each simulation, the simulation control passes the parameters to be used for the trial to the trial control, and asks the trial control to perform 
$k$ trials. Once complete, the trial control component returns the decisions made, $D_{s k i n}(k)$ and $D_{d r f m}(k)$. The performance metrics in Table 4.3 are then calculated for the $j^{\text {th }}$ simulation.

Table 4.3: Performance Metrics for the CRN Technique Simulation

\begin{tabular}{|c|l|l|}
\hline Symbol & Name & Description \\
\hline \hline$P_{D_{\text {skin }}}$ & Skin Probability of Detection & $\begin{array}{l}\text { Probability of detecting a skin re- } \\
\text { turn, given a skin return }\end{array}$ \\
\hline$P_{F A_{d r f m}}$ & DRFM false alarm probability & $\begin{array}{l}\text { Probability of detecting a DRFM } \\
\text { return, given a skin return }\end{array}$ \\
\hline$P_{D_{\text {drfm }}}$ & DRFM probability of detection & $\begin{array}{l}\text { Probability of detecting a DRFM } \\
\text { return, given a DRFM return }\end{array}$ \\
\hline$P_{N D_{d r f m}}$ & DRFM probability of no detection & $\begin{array}{l}\text { Probability of detecting a skin re- } \\
\text { turn, given a DRFM return }\end{array}$ \\
\hline$t_{D}$ & time to detection decision & $\begin{array}{l}\text { Time from first CRN pulse re- } \\
\text { ceived to outputting a final deci- } \\
\text { sion }\end{array}$ \\
\hline
\end{tabular}

These performance metrics attempt to follow standard radar conventions found in [9] and [10]. Recalling that $D=H_{0}=0$ or $D=H_{1}=1$, the calculation of each of these performance metrics is as follows:

$$
\begin{aligned}
P_{D_{s k i n}}(j) & =\frac{\sum_{i=0}^{k-1} D_{s k i n}(i)}{k} \\
P_{F A_{d r f m}}(j) & =1-P_{D_{s k i n}}(j) \\
P_{D_{\text {drfm }}}(j) & =\frac{\sum_{i=0}^{k-1} D_{d r f m}(i)}{k} \\
P_{N D_{d r f m}}(j) & =1-P_{D_{d r f m}}(j) \\
t_{D} & =n T_{P R I} N_{C P I}
\end{aligned}
$$


Once the simulation control has run $j$ simulations and calculated all of the performance metrics, it plots the results. When plotting the results, the $j$ data points along the $x$-axis represent the variable that was varied in this simulation. For example, Figure 4.13 shows the simulation results where the threshold was varied, therefore the

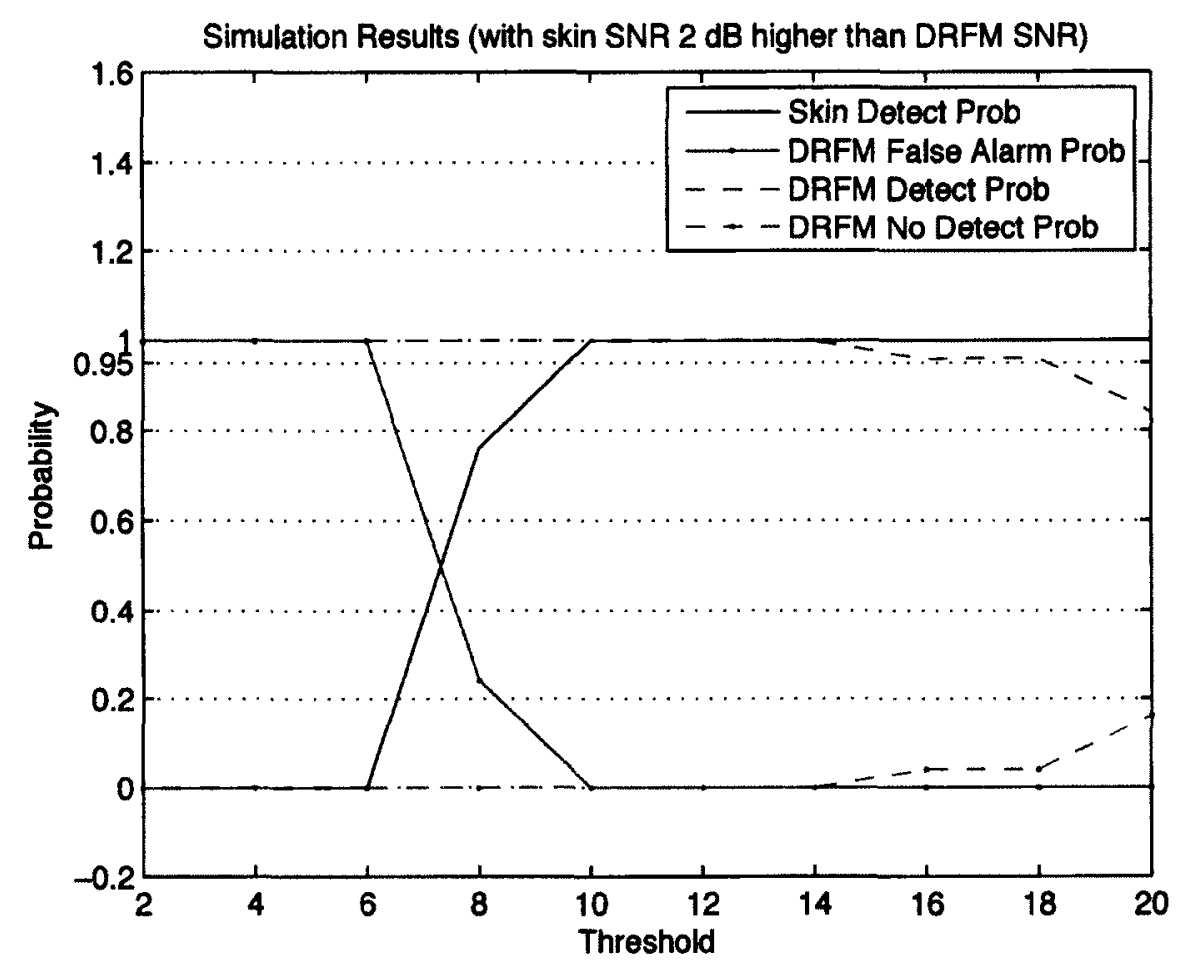

Figure 4.13: Example of a High Granularity Simulation Results Plot

$x$-axis represents $T$, while the $y$-axis represents probability. Henceforth, this style of simulation will be referred to as a high granularity simulation.

A low granularity simulation is also used quite often in Chapter 5 to determine appropriate parameter ranges for the high granularity simulations and also to show the effects of varying parameters at a conceptual level. Figure 4.14 is an example of a low granularity simulation. These simulations are visualized with a contour plot in which the $x$-axis of the graph is $T$, the $y$-axis is $S N R_{\text {radar }_{\text {okin }}}$ and the $z-$ axis is $P_{D_{\text {skin }}}+P_{D_{\text {drfm }}}$. In essence, this graph gives a general indication of what 


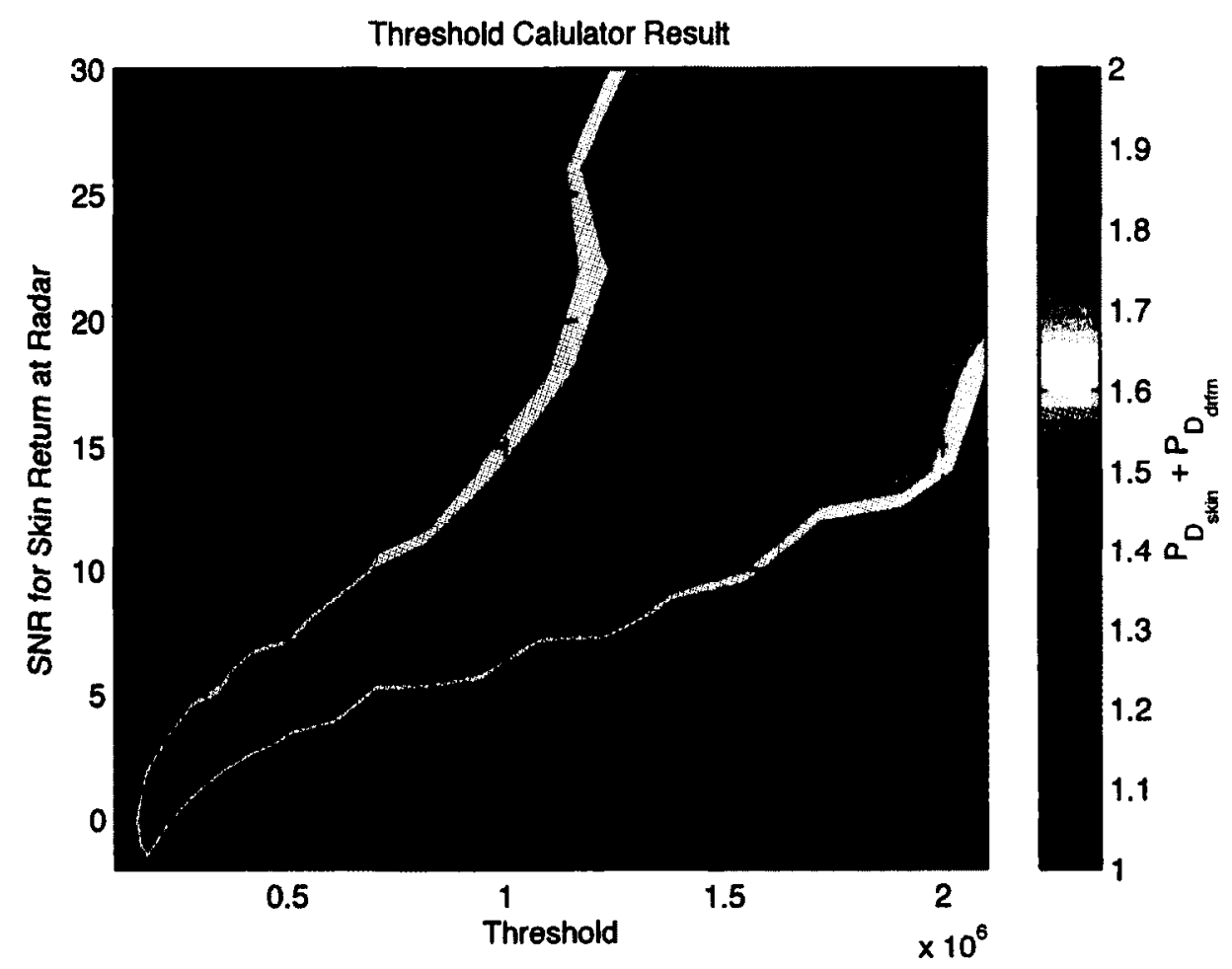

Figure 4.14: Example of a Low Granularity Simulation Results Plot

value of $T$ is required for a given $S N R_{\text {radar }_{s k i n}}$ and in the case of Figure 4.14, for $\triangle S N R=1 d B$. The ridge which has a value of 2 , represents the area where, for this particular simulation, $P_{D_{s k i n}}=1$ and $P_{D_{d r f m}}=1$.

To calculate $t_{P D}$, the value $n$ is taken from the $m$ of $n$ detector. In fact, the three parameters of (4.42) were not varied in Chapter 5. Taking the values from Table 4.2, $t_{D}=5 m s$ which will be the value for all simulations.

Chapter 5 will present the results in this format, with various parameter configurations of the $\mathrm{CRN}$ technique. 


\subsection{Summary}

This chapter has presented the CRN technique in concept and detail. It has discussed how incoherent sampling at the DRFM receiver causes a minute distortion of the originally transmitted CRN pulse. A PDF for the sampling delay that causes this distortion was derived. The EP Processor was presented as a system that is designed to detect the minute distortion caused by the DRFM jammer and is based on many commonly used radar components. The output of this system is an indicator of whether the target under investigation is a target or DRFM jammer. Finally, the simulation scheme was described and the performance metrics used to determine the system effectiveness were explained. 


\section{Chapter 5}

\section{Results}

This chapter will be organized in terms of the simulation parameters that were varied. In the scope of this thesis, it was not possible to vary every combination of parameters, therefore, the parameters that best demonstrate the capabilities and limitations of the CRN technique have been varied. These parameters are the CRN pulse length $N_{c r n}$, the CRN pulse bandwidth $B$, the center frequency of the modulator $\omega_{c}$, and the difference in SNRs, $\triangle S N R$. The different simulations will be measured against each other using the minimum achievable $S N R_{\text {radar }_{\text {skin }}}$. This will be defined as the minimum $S N R_{\text {radar }_{\text {skin }}}$ where the probability of detection of skin return is $P_{D_{s k i n}}>$ 0.95 and the probability of detection of a DRFM return is $P_{D_{d r f m}}>0.95$.

The simulations will be grouped at the highest level based on the length of the CRN pulse: standard, short and long. The standard length CRN pulse will be used to demonstrate the effects of varying $\triangle S N R$ and $\omega_{c}$. First, the required threshold $T$ will be found through experiment. Then, using that value of $T$, the effects of varying these parameters will be shown. The final section of the standard pulse simulations will vary $B$ and find the minimum achievable $S N R_{\text {radar }_{s k i n}}$ for each value of $B$ used. $\triangle S N R$ and $\omega_{c}$ will be held at their optimum values for simulation varying $B$, to allow for easy comparison. The following short and long pulse sections will include the $B$ varied simulations only, with the same fixed values of $\Delta S N R$ and $\omega_{c}$. Finally, all 
the minimum achievable $S N R_{\text {radar }_{s k i n}}$ values will be summarized in a table for easy comparison across the different CRN pulse lengths.

\subsection{Standard CRN Pulse}

The standard CRN pulse length will be set at $N_{c r n}=1200$ samples which corresponds to a CRN pulse duration of $T_{P D_{c r n}}=180 n s$. This standard pulse length will be used as a baseline for comparison and it will also be used to show the consequences of varying the $\triangle S N R$ and $\omega_{c}$ parameters.

\subsubsection{Baseline}

To set the baseline we will find the minimum achievable $S N R_{\text {radar }_{s k i n}}$ with the simulation using the parameters in Table 5.1. To find the minimum achievable SNR

Table 5.1: Simulation Parameters for Standard CRN Pulse Baseline

\begin{tabular}{|c|c|c|}
\hline Parameter & Low Granularity & High Granularity \\
\hline \hline$k$ & 25 & 50 \\
\hline$m$ & 3 & 3 \\
\hline$n$ & 5 & 5 \\
\hline$N_{C P I}$ & 10 & 10 \\
\hline$T$ & Varied & Varied \\
\hline$N_{\text {crn }}$ & 1200 samples & 1200 samples \\
\hline$\omega_{c}$ & 0.797 & 0.797 \\
\hline$B$ & 0.333 & 0.333 \\
\hline$S N R_{\text {radar }}$ skin & Varied & $-1 \mathrm{~dB}$ \\
\hline$\Delta S N R$ & $1 \mathrm{~dB}$ & $1 \mathrm{~dB}$ \\
\hline$S N R_{d r f m}$ & $40 \mathrm{~dB}$ & $40 \mathrm{~dB}$ \\
\hline$\frac{f_{s_{d}}}{f_{s_{r}}}$ & $\frac{11}{10}$ & $\frac{11}{10}$ \\
\hline
\end{tabular}


we first run a low granularity simulation that determines the threshold values, $T$,

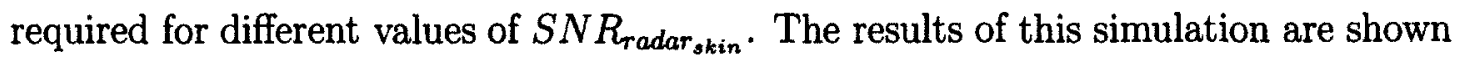
in Figure 5.1. The $x$-axis of the graph is $T$, the $y$-axis is $S N R_{\text {radar }_{s k i n}}$ and the $z$-axis is $P_{D_{s k i n}}+P_{D_{d r f m}}$. As mentioned earlier, we are using the measure of $P_{D_{s k i n}}>0.95$ and $P_{D_{\text {drfm }}}>0.95$ to determine minimum achievable $S N R_{\text {radar }}$ skin , which translates roughly to a $z$-axis value of $P_{D_{\text {skin }}}+P_{D_{\text {dr } f m}}>1$.9. From inspection of Figure 5.1 we

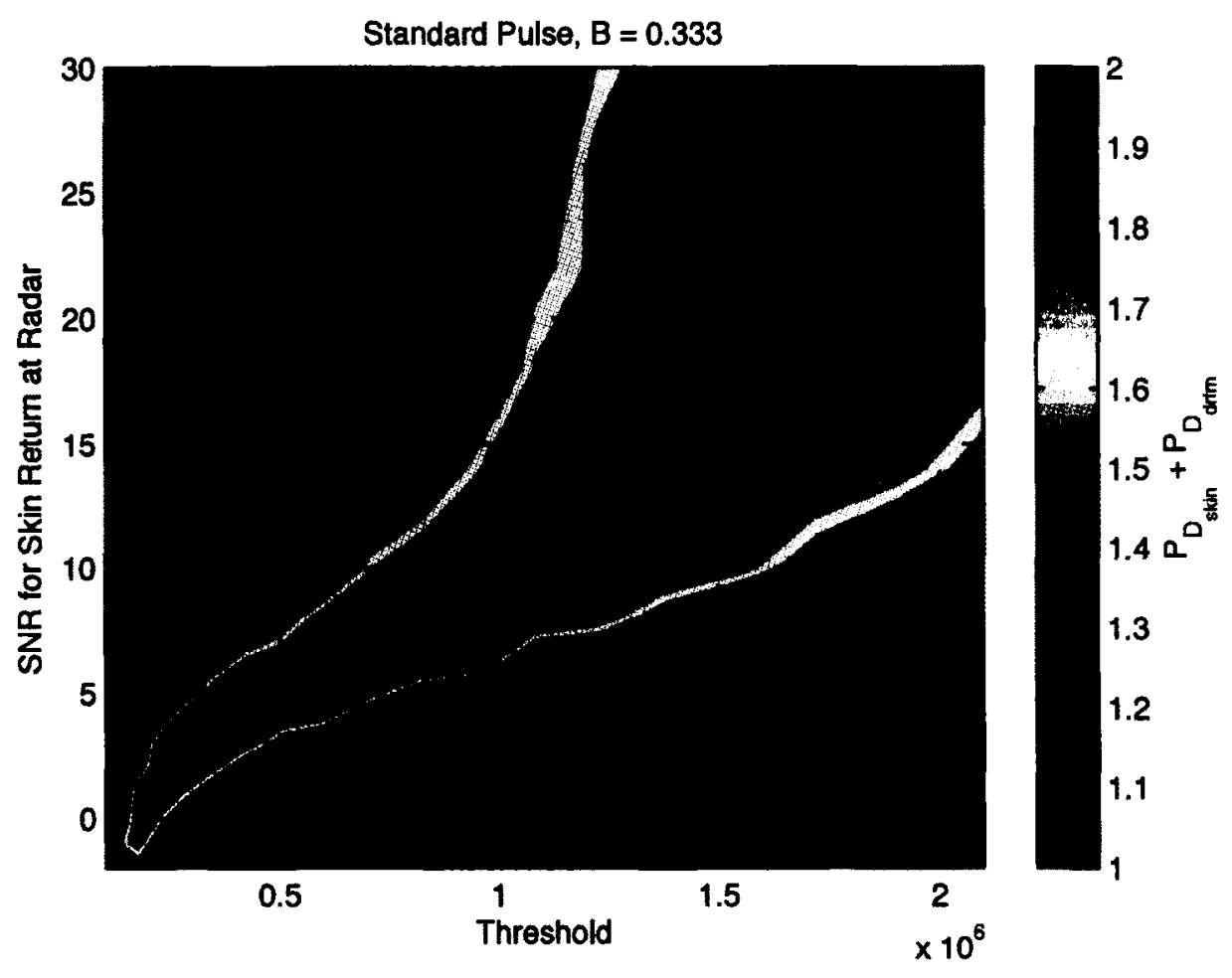

Figure 5.1: Standard CRN Pulse, Low Granularity, Baseline Threshold Calculation

can see that the minimum achievable SNR is approximately $-1 \mathrm{~dB}$ with $T \approx 180,000$. A high granularity simulation was then run with parameters from Table 5.1, varying $T$ around 180,000 to validate that $P_{D_{\text {skin }}}>0.95$ and $P_{D_{\text {drf } m}}>0.95$ is achievable at $S N R_{\text {radar }_{\text {skin }}}=-1 d B$. The result of this simulation is shown in Figure 5.2 and it can be seen that both probabilities of detection are approximately 0.98 . This result is recorded in the summary Table 5.6 for comparison purposes. 


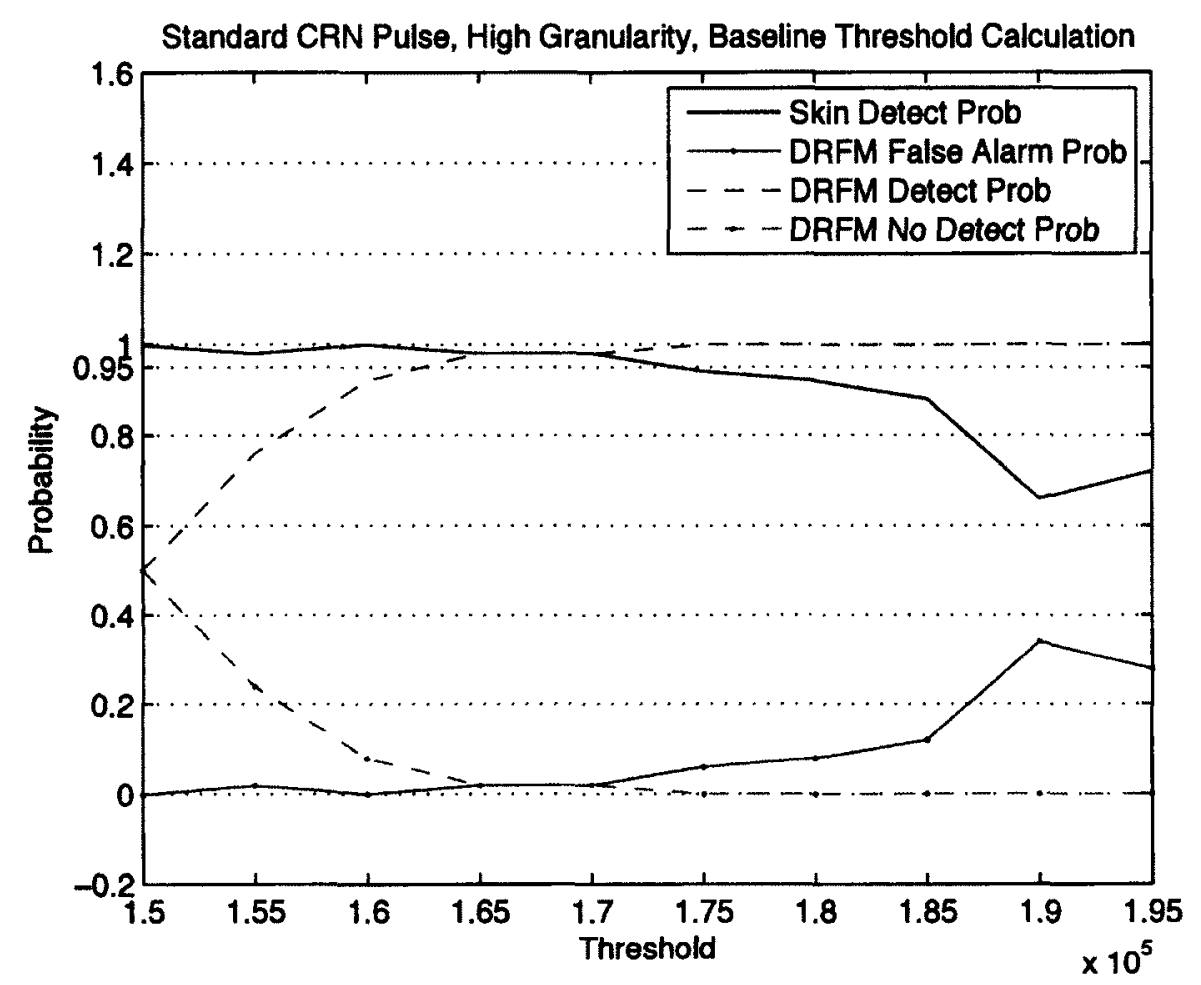

Figure 5.2: Standard CRN Pulse, High Granularity, Baseline Threshold Calculation $\left(S N R_{\text {radar }_{\text {skin }}}=-1 d B\right)$

In actual operation, the threshold $T$ would need to be pre-defined yet variable based on operating conditions. Since the ridge of high probability in Figure 5.1 is smooth and uniform, it should be quite easy to find a function that would determine an appropriate threshold based on $S N R_{\text {radar }_{s k i n}}$. However, the difficult part may be accurately measuring the value of $S N R_{\text {radar }_{s k i n}}$ for this purpose. This is addressed as an open area of research, see Section 6.2.1.

Now that a baseline is set, we will vary some parameters with low granularity simulations to demonstrate the effects of those variables on the CRN technique. 


\subsection{2 $\triangle$ SNR Varied}

The first parameter that will be investigated is $\triangle S N R$, the difference in SNR between the skin return and DRFM return at the radar receiver - an important parameter. Typically a DRFM wants its return to overpower the skin return, which is one of many ways the DRFM jammer attempts to attract the radar onto its jamming signal. If the DRFM return has much more power than the skin return, the radar receiver may be able to detect the presence of the jammer through AGC alone. Therefore, the DRFM jammer tries to keep its return a few $\mathrm{dB}$ higher than the skin return. This simulation will provide results for $\triangle S N R=1 d B, 2 d B, \& 3 d B$, using the same base parameters from Table 5.1. The effect of increasing $\triangle S N R$ can be easily seen in the graphs of Figure 5.3. The minimum achievable $S N R_{\text {radar }_{\text {skin }}}$ rises as $\triangle S N R$ rises, an expected result. Another evident and also promising result that can be seen in Figure 5.3 is that the value of $T$ does not change as a result of varying $\triangle S N R$. This will make an algorithm to adaptively set a threshold easier to implement.

Figure 5.4 shows the effects of varying $\triangle S N R$ with $T=167,500$ and $S N R_{\text {radar }_{\text {skin }}}=-1 d B$ in high granularity. It can be seen that even with $S N R_{\text {radar }_{\text {skin }}}=-1 d B$ and $\triangle S N R=2 d B$, it nearly reaches the requirements for $-1 d B$ to be declared the minimum achievable $S N R_{\text {radar }_{\text {skin }}}$.

In the end, the minimum achievable $S N R_{\text {radar }_{\text {skin }}}$ for different values of $\triangle S N R$ are given in Table 5.2. The consequence of these results on the CRN technique is

Table 5.2: Summary of Results for $\triangle S N R$ Varied

\begin{tabular}{|c|c|}
\hline$\Delta S N R$ & Minimum Achievable $S N R_{\text {radar }_{\text {akin }}}$ \\
\hline \hline $1 d B$ & $-1 d B$ \\
\hline $2 d B$ & $0 d B$ \\
\hline $3 d B$ & $2 d B$ \\
\hline
\end{tabular}

that it will be effective for SNR values as low as those in the second column of Table 
(a) Result for $\triangle S N R=1 d B$

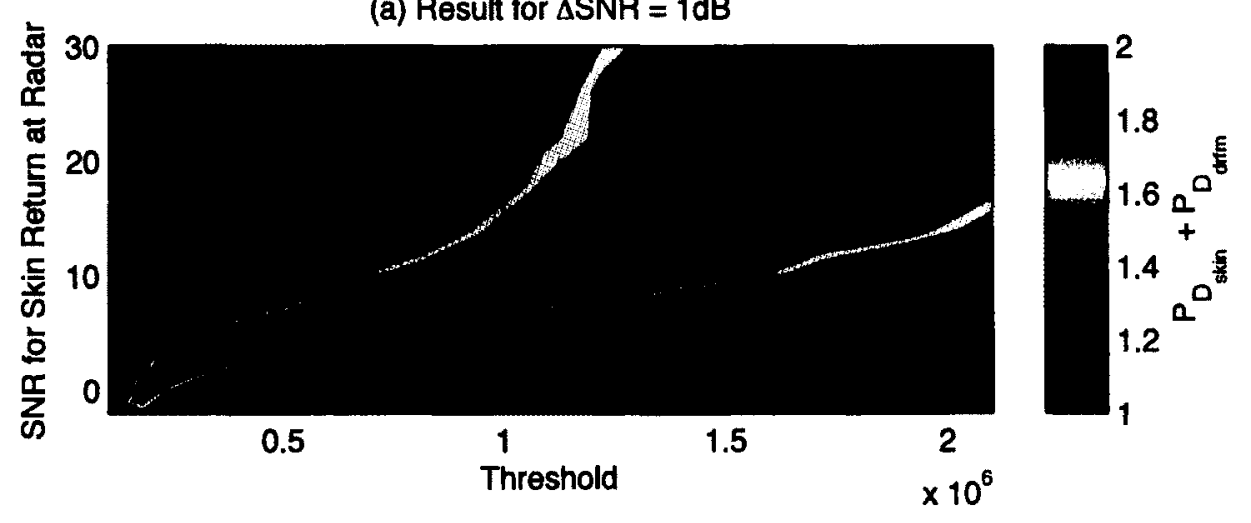

(b) Result for $\triangle S N R=2 \mathrm{~dB}$

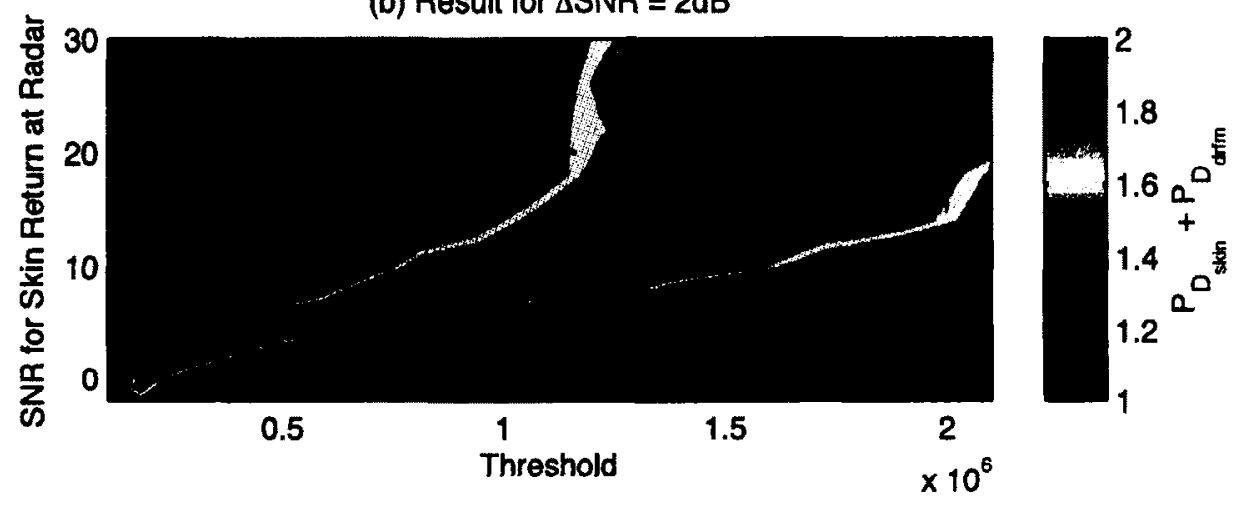

(c) Result for $\triangle S N R=3 \mathrm{~dB}$

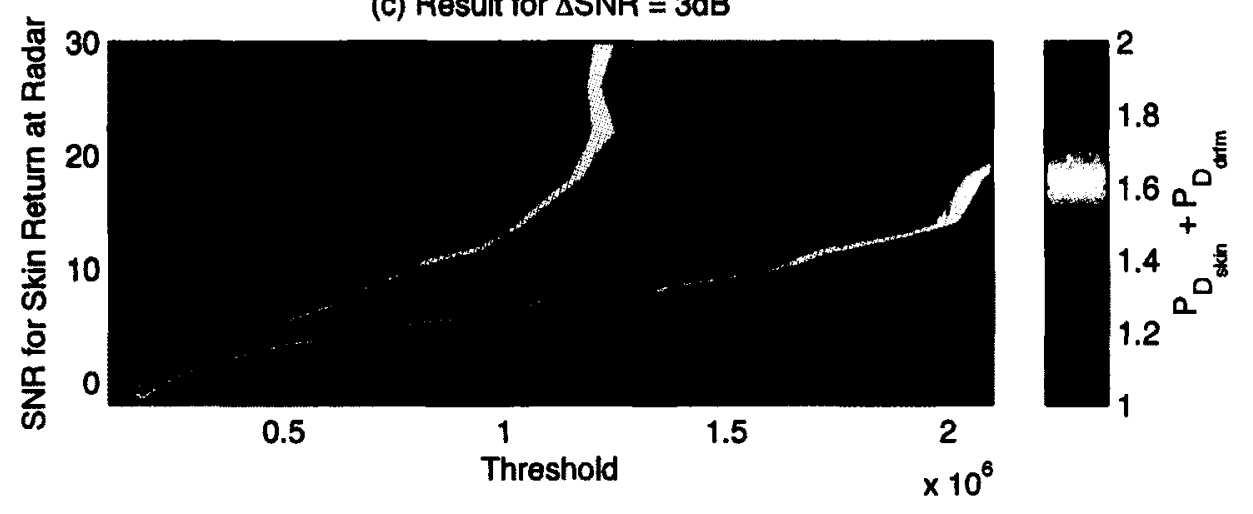

Figure 5.3: Standard CRN Pulse, Low Granularity, Effect of Varying $\triangle S N R$ (a) $\triangle S N R=1 d B$, (b) $\triangle S N R=2 d B$, (c) $\triangle S N R=3 d B$

5.2. When the DRFM begins to increase the power to values where $\Delta S N R>3 d B$, it may be more effective to use EP techniques based on AGC. 


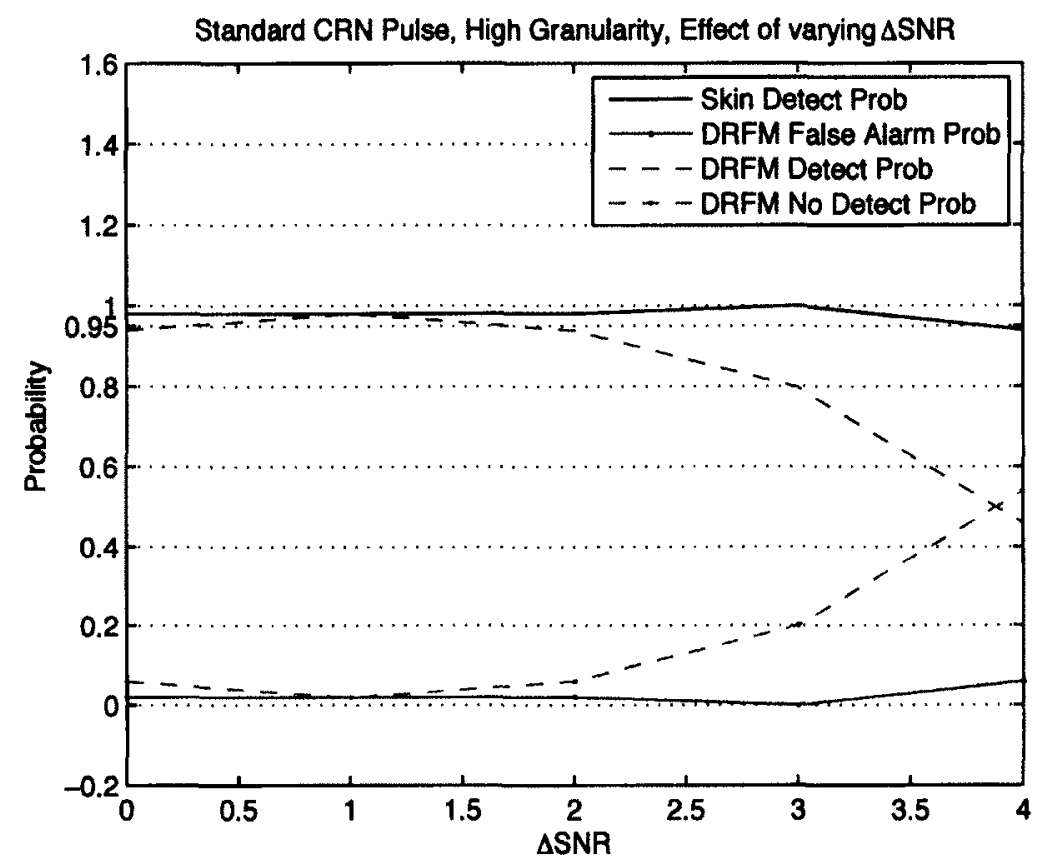

Figure 5.4: Standard CRN Pulse, High Granularity, Effect of Varying $\Delta S N R$

\subsubsection{Center Frequency $\left(\omega_{c}\right)$ Varied}

The second parameter that will be varied is $\omega_{c}$, the center frequency of the modulator and BPFs. This parameter plays an important role in the CRN technique. As Figure 5.5 shows the higher the value of the $\omega_{c}$, the higher the effectiveness of the CRN technique. The simulation used to create Figure 5.5 used the base parameters from Table 5.1 and varied $\omega_{c}$ with values of $0.8,0.7$ and 0.6 . The undesirable results for $\omega_{c}=0.6$ are attributed to sampling the random noise of the CRN pulse at a comfortable rate, much lower than the Nyquist rate. The result here shows that the CRN technique is much more effective when the CRN pulse is sampled as close to the Nyquist rate as possible. At this rate, the CRN pulse is most distorted by the DRFM due to incoherent sampling as explained in Sections 2.2.1 and 4.1.1. 

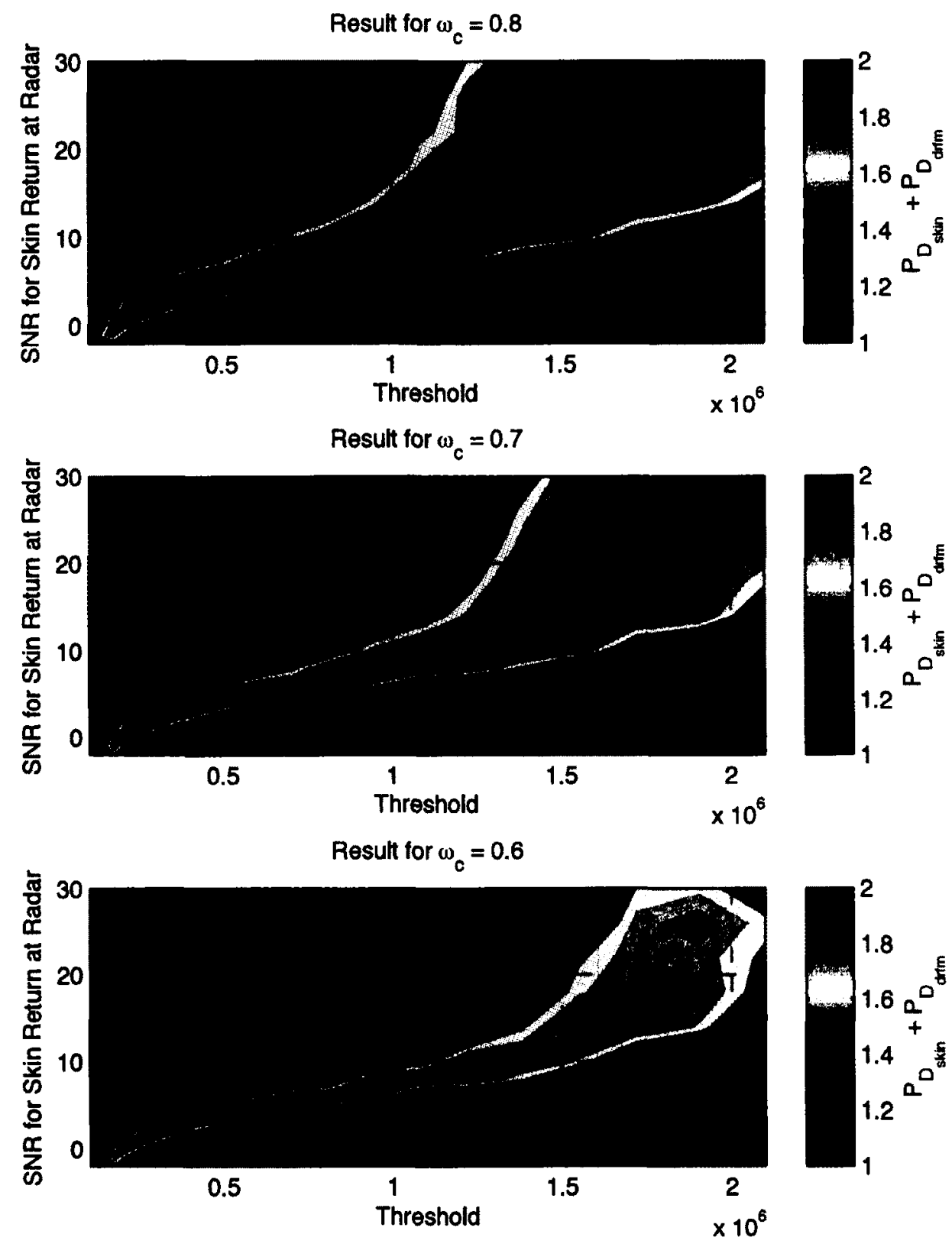

Figure 5.5: Standard CRN Pulse, Low Granularity, Effect of Varying $\omega_{c}$ (a) $\omega_{c}=0.8$, (b) $\omega_{c}=0.7$, (c) $\omega_{c}=0.6$

The consequence on the CRN technique is that $\omega_{c}$ is always set such that:

$$
\begin{aligned}
\omega_{c}+\frac{B}{2}+g & =1 \\
\omega_{c} & =1-\frac{B}{2}-g
\end{aligned}
$$


where $B$ is the bandwidth of the CRN technique and $g$ is a guard value that ensures that filter stop bands can be implemented before they reach $\frac{f_{s}}{2}$. For the simulations presented in this chapter, $g=0.036$. In the next section, as $B$ is varied, Equation (5.1) is applied to find $\omega_{c}$.

\subsubsection{Bandwidth $(B)$ Varied}

Figure 5.6 shows the effect of varying $B$ for a standard CRN pulse. Please note that the $x$-axis and $z$-axis scales vary between the sub-figures for the remainder of low granularity figures in the chapter. Also, in Figure 5.6(b) it appears that as $S N R_{\text {radar }_{\text {skin }}}$ rises, the probabilities of detection decrease. This is not the case. The ridge of high probability has narrowed compared to Figure 5.6(a) and the sampling points on the $x$-axis are too sparse to completely define the ridge. This occurred because the sampling points used were of an exponential function to reduce the computation time from days to hours. The problem was not encountered in Figure 5.1(c) because the span of threshold values was less.

Originally, it was a goal to demonstrate this technique at bandwidth values of $B=0.333, B=0.2$ and $B=0.1$ in normalized frequency. However, simulations at $B=0.1$ did not yield favourable results. After some inspection, it was discovered that noise in the band of interest was being generated by the demodulator and possibly being multiplied by the low pass filter. This noise was overpowering the received CRN pulse portion of the signal and causing the matched filter to miss the CRN pulse completely. It was decided to defer this issue as future research and simply simulate at $B=0.15$. Some possible solutions can be found in Section 6.2.5.

In any case, Figure 5.6 shows that as the bandwidth is narrowed the minimum achievable SNR becomes higher. Figure 5.7 shows high granularity simulations for each value of $B$, at the minimum achievable SNR. The results gleaned from Figure 5.7 are found in Table 5.3. These values are further summarized and compared to 

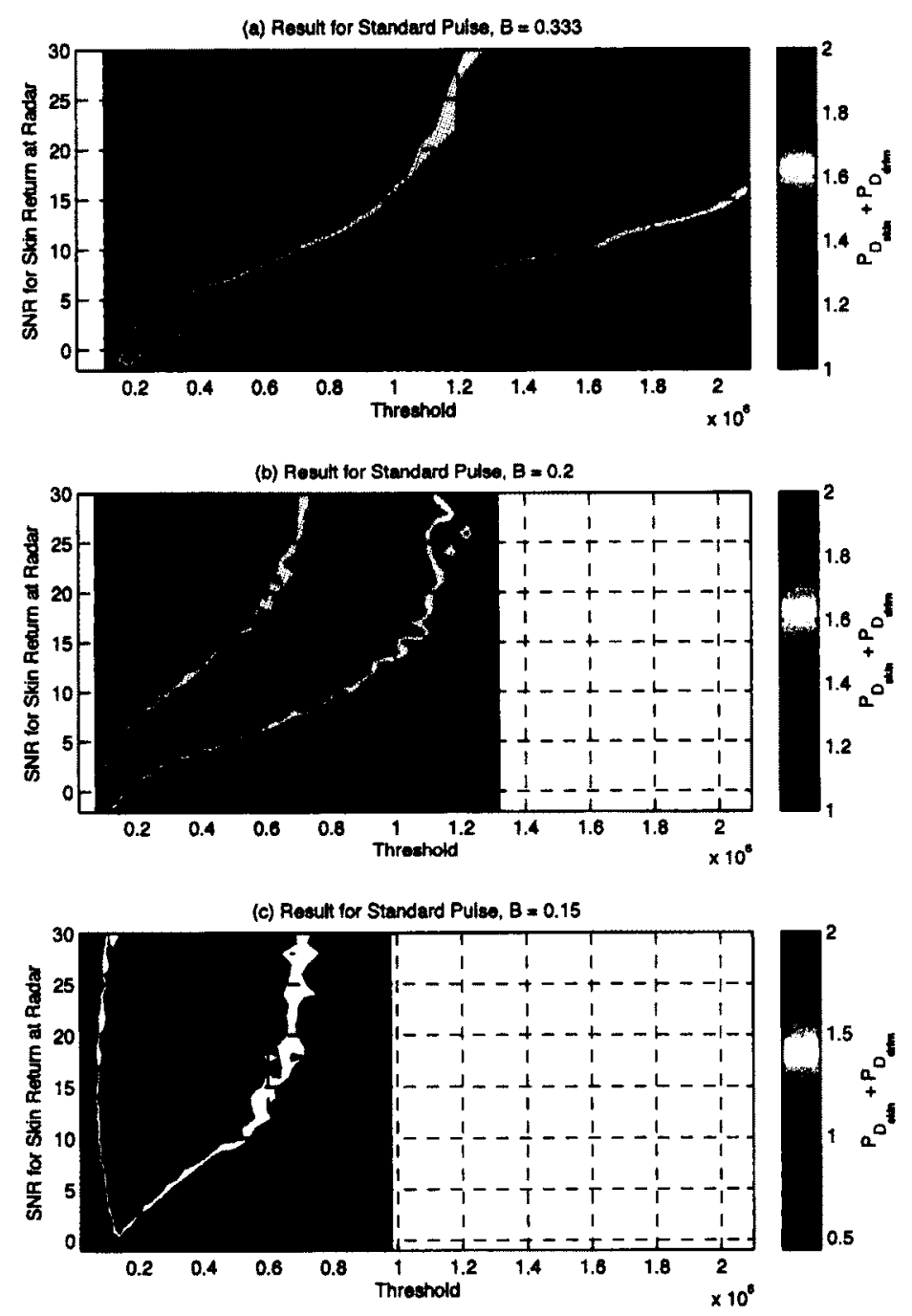

Figure 5.6: Standard CRN Pulse, Low Granularity, Effect of Varying $B$ (a) $B=$ 0.333 , (b) $B=0.2$, (c) $B=0.15$

other CRN pulse lengths in Table 5.6.

The next two sections will follow the same methodology used above to vary $B$ for short and long CRN pulses. The results will be presented with less commentary and a comparison of the results for different pulse lengths will follow in the chapter summary. 
Table 5.3: Summary of Results for Standard CRN Pulse, $B$ Varied

\begin{tabular}{|c|c|c|c|c|c|}
\hline Sub-figure & $B$ & $T$ & $S N R_{\text {radar }_{\text {skin }}}$ & $P_{D_{\text {skin }}}$ & $P_{D_{\text {dr } f m}}$ \\
\hline \hline (a) & 0.333 & 180,000 & $-1 d B$ & 0.98 & 0.98 \\
\hline (b) & 0.2 & 129,000 & $0 d B$ & 1 & 1 \\
\hline (c) & 0.15 & 140,000 & $2 d B$ & 1 & 0.98 \\
\hline
\end{tabular}




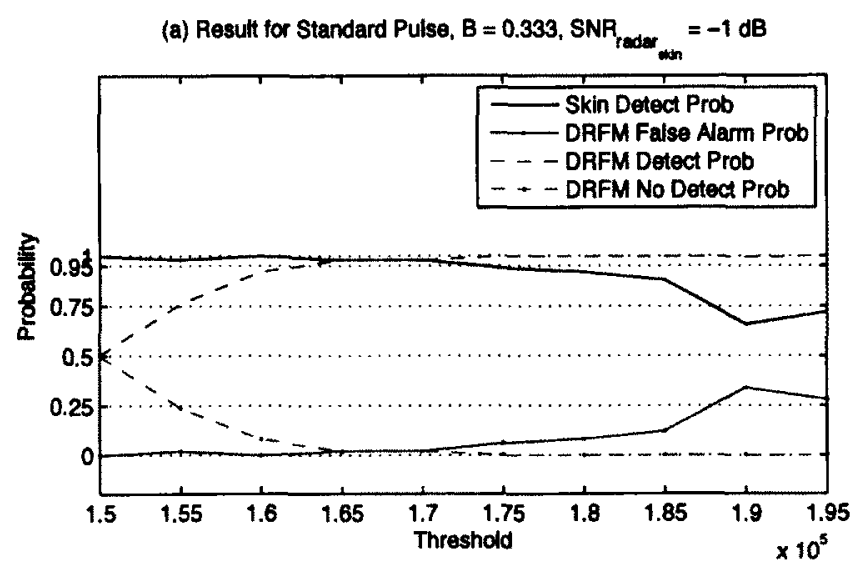

(b) Result for Standard Pulse, B $=0.2, S_{\text {redow }}=0 \mathrm{~dB}$

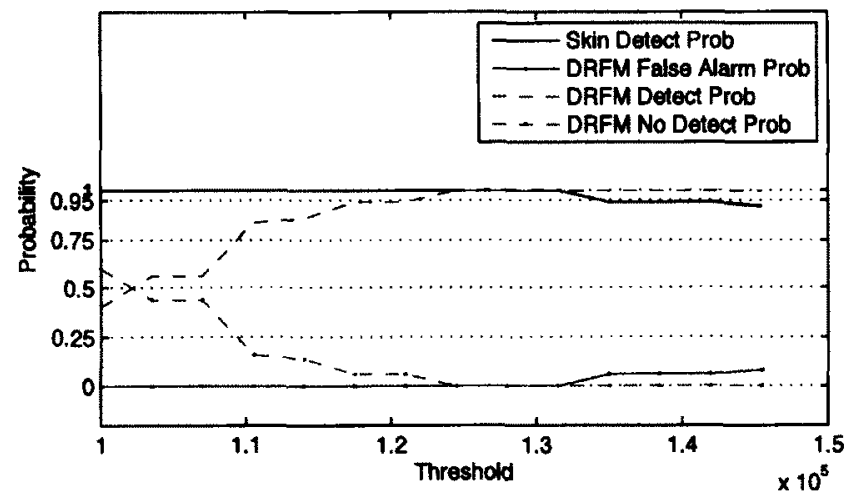

(c) Result for Standard Pulse, $B=0.15$, SNR $_{\text {reder }}=2 \mathrm{~dB}$

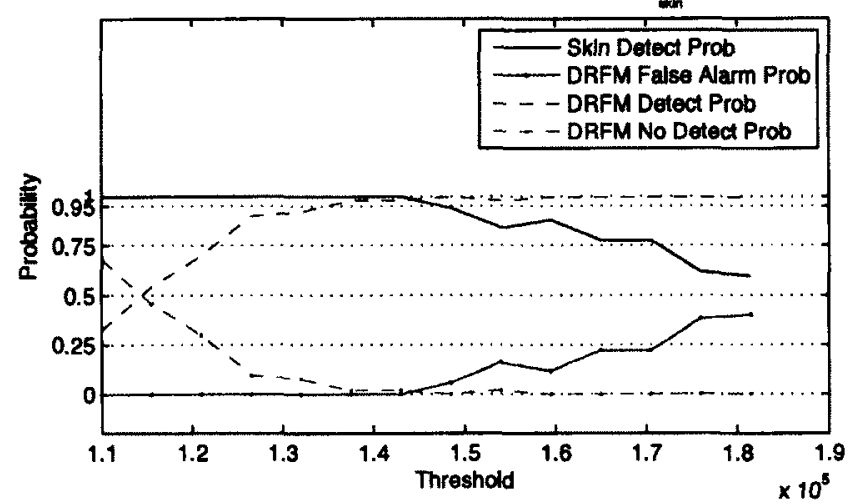

Figure 5.7: Standard CRN Pulse, High Granularity, Effect of Varying $B$ at Minimum Achievable SNR

(a) $B=0.333$ and $S N R_{\text {radar }_{\mathrm{skin}}}=-1 \mathrm{~dB}$,

(b) $B=0.2$ and $S N R_{\text {radar }_{\text {skin }}}=0 d B$,

(c) $B=0.15$ and $S N R_{\text {radar }_{s k i n}}=2 d B$ 


\subsection{Short CRN Pulse}

This section will present the results for the short CRN pulse with varied $B$ values. The short CRN pulse length used $N_{c r n}=600$ samples, which corresponds to a CRN pulse duration of $T_{P D_{\text {crn }}}=90 \mathrm{~ns}$. This shorter pulse is included because there may
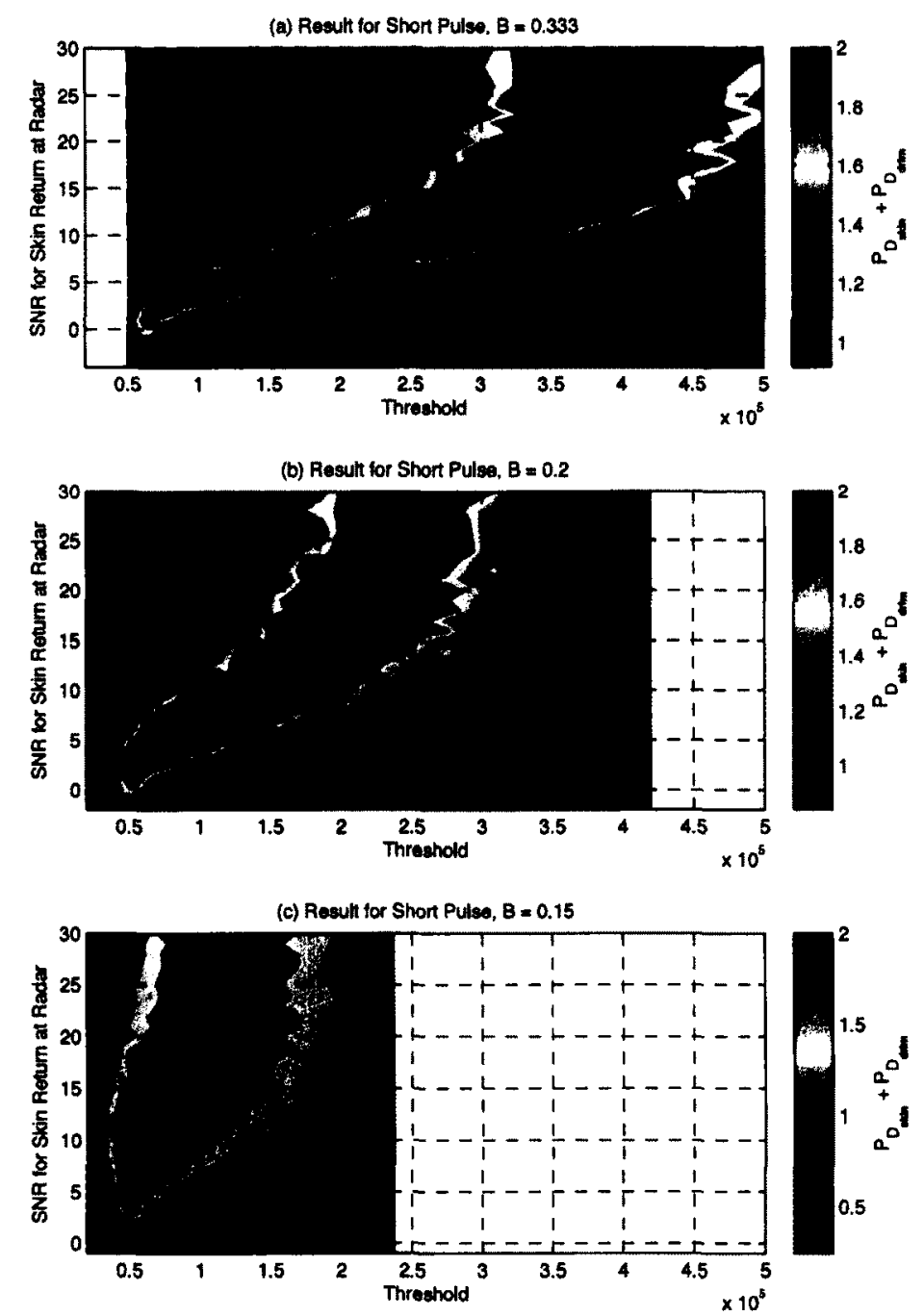

Figure 5.8: Short CRN Pulse, Low Granularity, Effect of Varying $B$ (a) $B=0.333$, (b) $B=0.2$, (c) $B=0.15$ 
be situations when it could be useful, such as: (1) when a radar is heavily scheduled and it can only afford a short CRN pulse, (2) when the radar pulse itself is short and a shorter CRN pulse is required as well, or (3) when SNRs are high enough that a shorter CRN pulse will not affect performance.

Figure 5.8 provides a low granularity view of the effects of varying $B$ using a short CRN pulse. The effects remain the same but may be slightly more acute compared to a standard pulse; the minimum achievable SNR increases as the bandwidth decreases. Figure 5.9 shows the high granularity simulations for each value of $B$ and the data points taken are shown in Table 5.4.

Table 5.4: Summary of Results for Short CRN Pulse, $B$ Varied

\begin{tabular}{|c|c|c|c|c|c|}
\hline Sub-figure & $B$ & $T$ & $S N R_{\text {radar }_{\text {skin }}}$ & $P_{D_{\text {skin }}}$ & $P_{D_{\text {drfm }}}$ \\
\hline \hline (a) & 0.333 & 67,600 & $1 d B$ & 0.98 & 1 \\
\hline (b) & 0.2 & 50,800 & $0 d B$ & 1 & 1 \\
\hline (c) & 0.15 & 51,700 & $5 d B$ & 0.99 & 0.99 \\
\hline
\end{tabular}




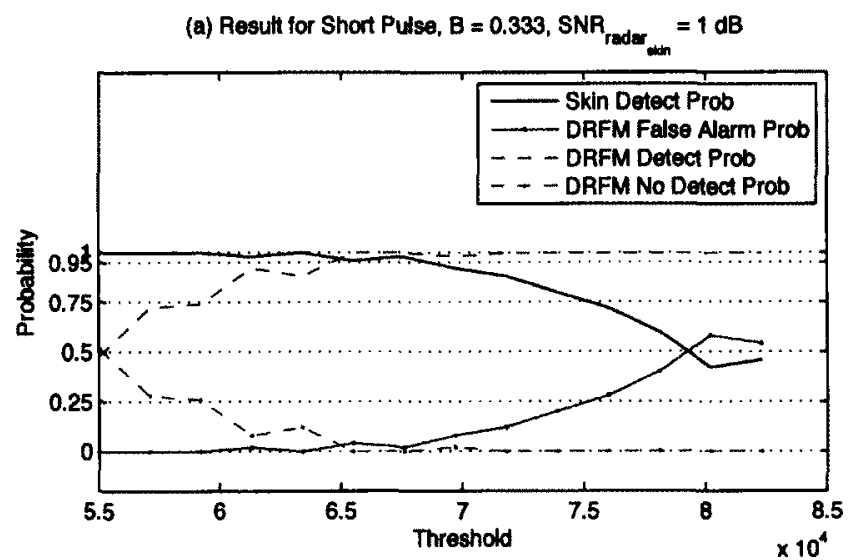

(b) Result for Short Pulse, $B=0.2$, SNR $_{\text {rader }}=1 \mathrm{~dB}$

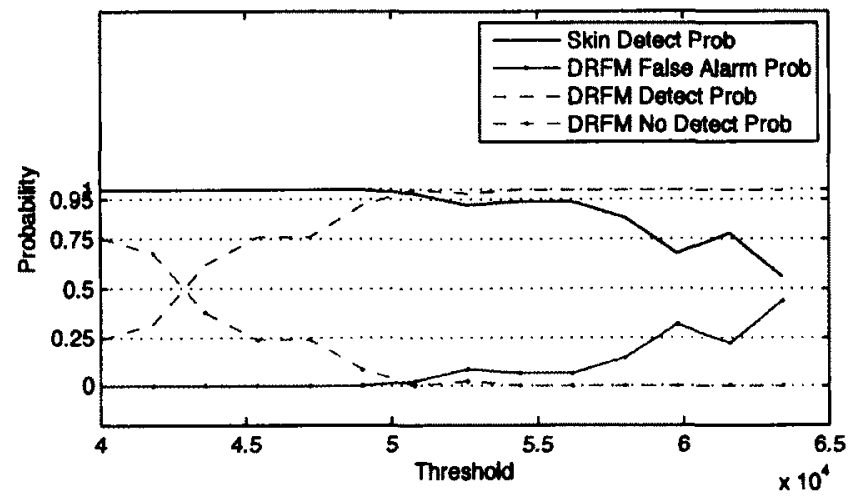

(c) Result for Short Pulse, B $=0.15, \mathrm{SNR}_{\text {rader }}=5 \mathrm{~dB}$

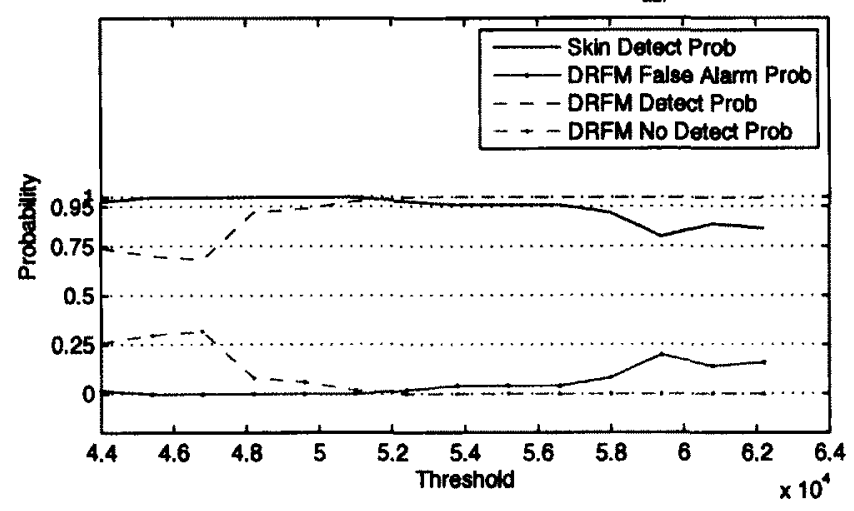

Figure 5.9: Short CRN Pulse, High Granularity, Effect of Varying $B$ at Minimum Achievable SNR

(a) $B=0.333$ and $S N R_{\text {radar }_{\text {skin }}}=1 \mathrm{~dB}$,

(b) $B=0.2$ and $S N R_{\text {radar }_{\text {skin }}}=1 \mathrm{~dB}$,

(c) $B=0.15$ and $S N R_{\text {radar }_{\text {skin }}}=5 d B$ 


\subsection{Long CRN Pulse}

This section will present the results for the long CRN pulse with $B$ varied. The long CRN pulse length used has $N_{c r n}=2400$ samples which corresponds to a CRN pulse duration of $T_{P D_{c r n}}=360 \mathrm{~ns}$ at the assumed sampling frequency $f_{s}=6.667 \mathrm{GHz}$.

There are situations where a radar may have to detect distant targets equipped with a DRFM jammer and the longer CRN pulse allows for reliable detection of skin and DRFM returns at lower SNRs. Another situation where the long CRN pulse would be useful is when a radar needs higher probabilities of detection or equivalently, lower false alarm rates. The longer pulse provides more sampling points and more power to the CRN pulse which creates a more sensitive matched filter and more effective detection.

Following the same methodology as before, Figure 5.10 provides low granular graphs of the $P_{D_{s k i n}}$ and $P_{D_{d r f m}}$ when $T$ and $S N R_{\text {radarskin }}$ are varied. From each subfigure, corresponding to a certain value of $B$, the rough minimum achievable SNR and threshold value can be gleaned for use in the high granularity simulations.

Figure 5.11 shows this high granularity analysis which is summarized in Table 5.5.

Table 5.5: Summary of Results for Long CRN Pulse, $B$ Varied

\begin{tabular}{|c|c|c|c|c|c|}
\hline Sub-figure & $B$ & $T$ & $S N R_{\text {radar }_{\text {skin }}}$ & $P_{D_{\text {skin }}}$ & $P_{D_{\text {drfm }}}$ \\
\hline \hline (a) & 0.333 & 345,000 & $-3 d B$ & 0.95 & 0.95 \\
\hline (b) & 0.2 & 325,000 & $-2 d B$ & 1 & 0.98 \\
\hline (c) & 0.15 & 323,000 & $-1 d B$ & 0.95 & 0.95 \\
\hline
\end{tabular}

The observations of Figure 5.10 are the same for the low granularity graphs of the other CRN pulse lengths; as bandwidth narrows the minimum achievable SNR rises. 

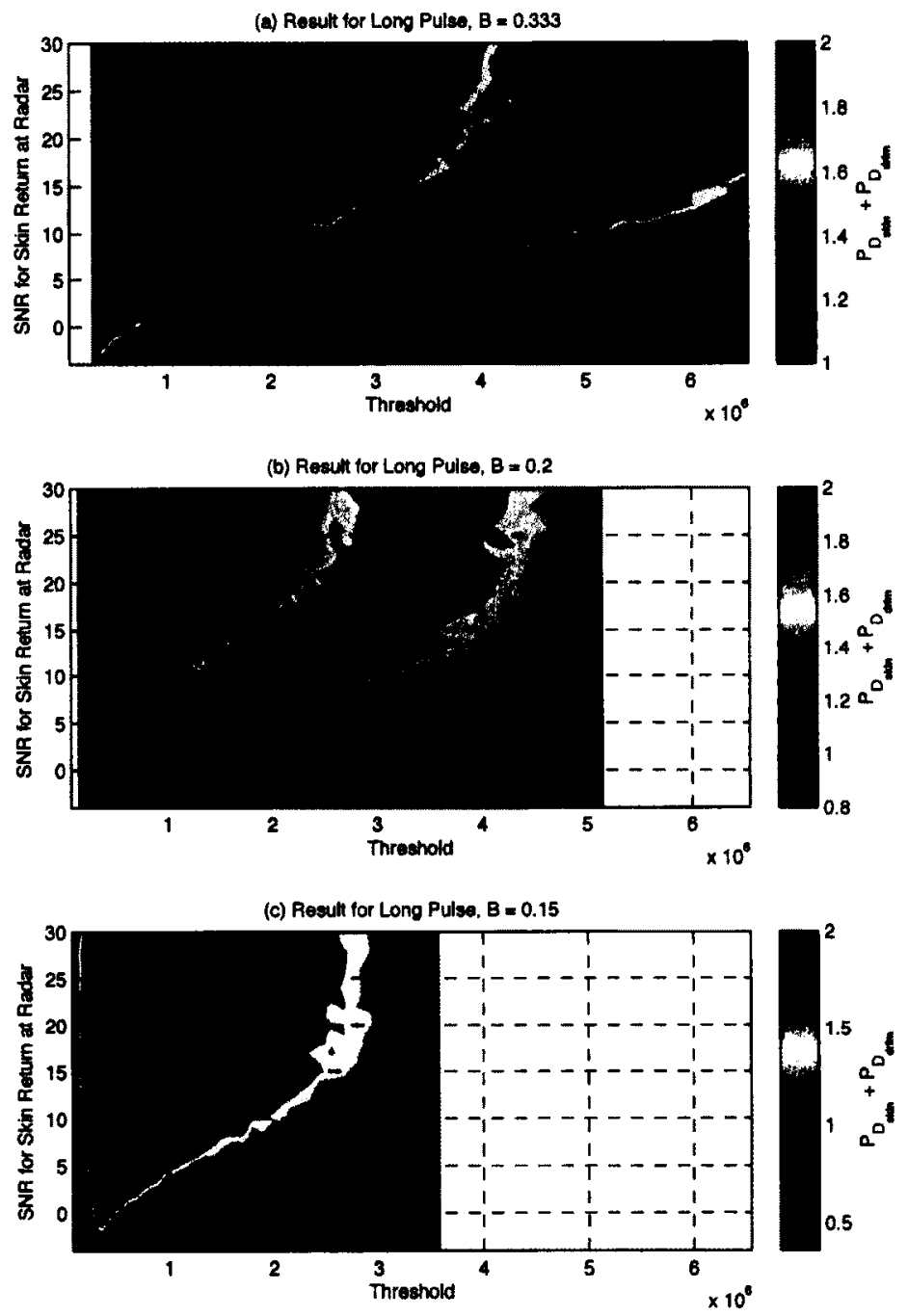

Figure 5.10: Long CRN Pulse, Low Granularity, Effect of Varying $B$ (a) $B=0.333$, (b) $B=0.2$, (c) $B=0.15$ 

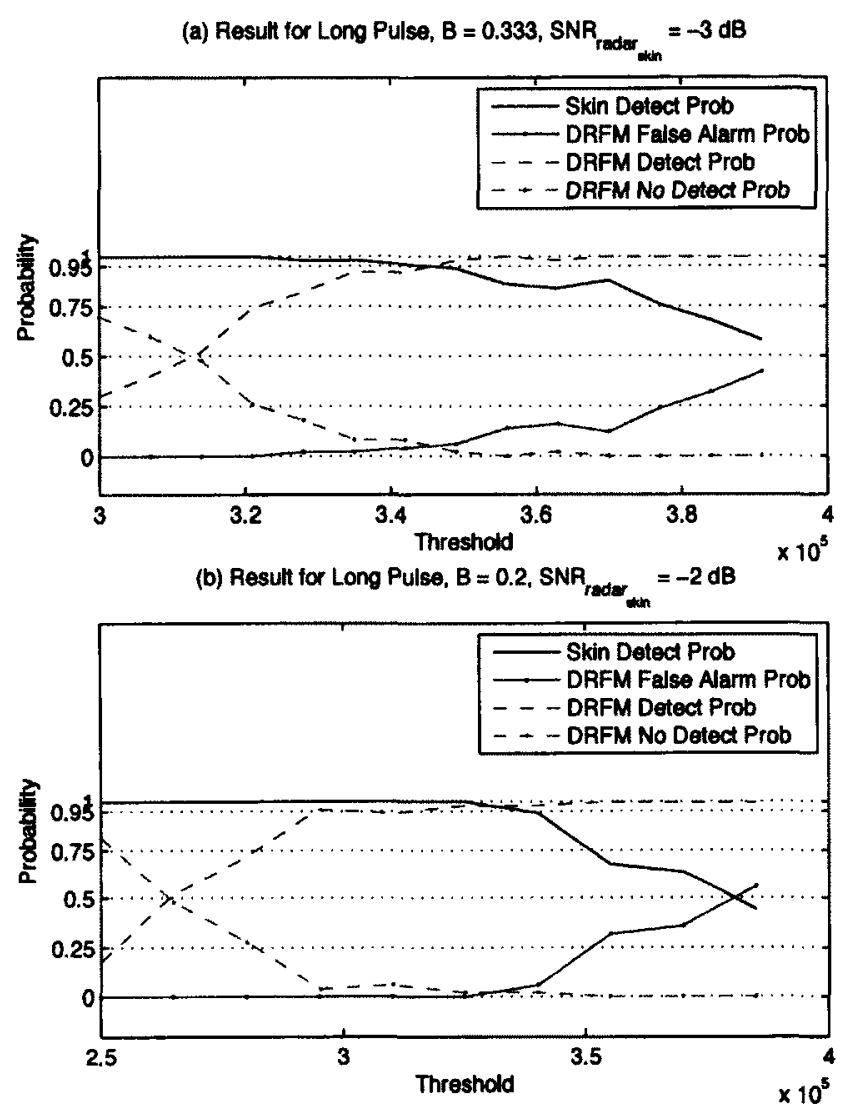

(c) Result for Long Pulse, B $=0.15$, SNR $_{\text {rade }}=-1 \mathrm{~dB}$

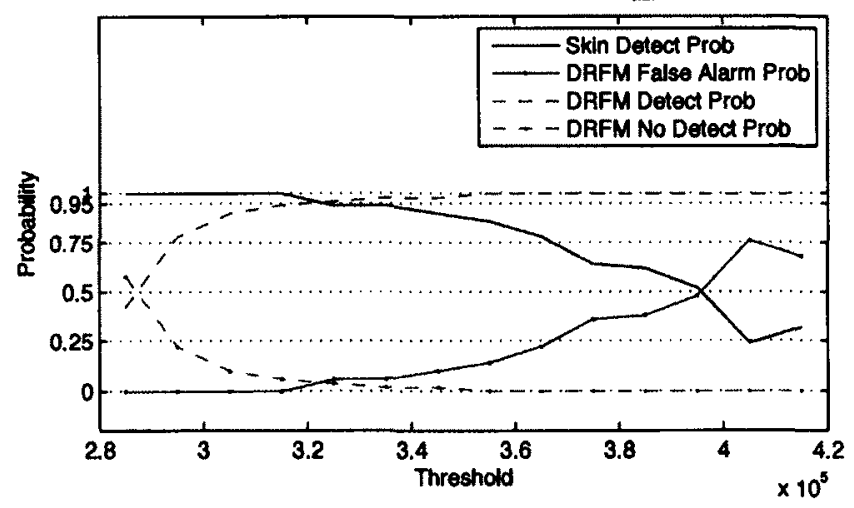

Figure 5.11: Long CRN Pulse, High Granularity, Effect of Varying $B$ at Minimum Achievable SNR

(a) $B=0.333$ and $S N R_{\text {radar }_{\text {skin }}}=-3 d B$,

(b) $B=0.2$ and $S N R_{\text {radar }_{\text {skin }}}=-2 d B$,

(c) $B=0.15$ and $S N R_{\text {radar }_{\text {skin }}}=-1 d B$ 


\subsection{Summary}

This chapter has presented and analysed many results from varying a subset of parameters in the simulation of the CRN technique. The subset of parameters were chosen as they were expected to have the strongest influence on the performance of the CRN technique. There is a requirement to simulate the CRN technique varying the full set of parameters and to optimize the CRN technique as a result. However, this task was beyond the scope of this thesis. Some important results were collected from the few parameters that were varied.

First, it was discovered that $\Delta S N R$, the difference in SNR between a skin return and DRFM return at the radar receiver, does not have a great impact on the performance of the CRN technique. The relationship is basically $1: 1$, if $\Delta S N R$ increases by one, the minimum achievable $S N R_{\text {radarsin }_{\text {ski }}}$ is also raised by one. However, if the DRFM attempts to simply over power the skin return by more than $3 \mathrm{~dB}$, the radar has the advantage because it can use existing EP techniques based on AGC. This forces the DRFM into the range of $0 d B<\triangle S N R<3 d B$, where the CRN technique is effective. Also, it was observed that varying $\triangle S N R$ had little to no effect on $T$. This means that any adaptive threshold algorithm would not need to consider $\Delta S N R$-a very favourable observation.

Second, a very clear conclusion can be drawn from the simulations varying $\omega_{c}$. The random noise of the CRN pulse must be as close as possible to the Nyquist rate to be most effective. This is intuitive based on the background given in Section 2.2.1, signals incoherently sampled close to the Nyquist rate will be more distorted than those sampled with a higher sampling frequency. This led to fixing $\omega_{c}$ with Equation (5.1) for the remaining simulations varying bandwidth.

Finally, a summary of the minimum achievable $S N R_{\text {radar }_{s k i n}}$ results recorded from 
Table 5.6: Summary of CRN Technique Simulation Results

\begin{tabular}{|c|c|c|c|c|c|c|c|}
\hline & \multicolumn{2}{|c|}{ Short Pulse } & \multicolumn{2}{|c|}{ Standard Pulse } & \multicolumn{2}{|c|}{ Long Pulse } \\
\hline & & $N_{c r n}$ & $T_{P D_{c r n}}$ & $N_{c r n}$ & $T_{P D_{c r n}}$ & $N_{c r n}$ & $T_{P D_{\text {crn }}}$ \\
\hline$\omega_{c}$ & $B$ & 600 samp & $90 \mathrm{~ns}$ & 1200 samp & $180 \mathrm{~ns}$ & $2400 \mathrm{samp}$ & $360 \mathrm{~ns}$ \\
\hline 0.79 & 0.333 & \multicolumn{2}{|c|}{$1 \mathrm{~dB}$} & \multicolumn{2}{|c|}{$-1 \mathrm{~dB}$} & \multicolumn{2}{|c|}{$-3 \mathrm{~dB}$} \\
\hline 0.86 & 0.2 & \multicolumn{2}{|c|}{$1 \mathrm{~dB}$} & \multicolumn{2}{|c|}{$0 \mathrm{~dB}$} & \multicolumn{2}{|c|}{$-2 \mathrm{~dB}$} \\
\hline 0.88 & 0.15 & \multicolumn{2}{|c|}{$5 \mathrm{~dB}$} & \multicolumn{2}{|c|}{$2 \mathrm{~dB}$} & \multicolumn{2}{|c|}{$-1 \mathrm{~dB}$} \\
\hline
\end{tabular}

varying $B$ and $N_{c r n}$ are presented in Table 5.6. On the surface, it is clear that the long pulse will provide the best performance. It also allows for a reduced bandwidth with the smallest increase in minimum achievable SNR. Through observation, the minimum achievable SNR is most likely related to the bandwidth-time product, $B T_{P D_{\text {crn }}}$. Figure 5.12 shows the relationship between minimum achievable SNR and $B T_{P D_{c r n}}$ with both a linear and quadratic trend line. It is thought that the quadratic trend line is more accurate. There are two comments to be made of this resultant relationship which will be important for the radar designer attempting to incorporate the CRN technique.

First, it appears in Figure 5.12 that there is likely a point of diminishing returns around a value of $B T_{P D_{c r n}}=50$, but more data needs to be collected at lower and higher values of $B T_{P D_{c r n}}$. At some point, as a higher percentage of the frequency components are closer to the Nyquist rate, the incoherent sampling by the radar receiver will cause too much distortion of both the skin and DRFM return, and neither will be reliably detected. Once an adaptive threshold algorithm is implemented, more research could determine if there is a point of diminishing return and what it may be.

Second, this relationship gives the radar designer a tool to choose the appropriate $B$ and $N_{\text {crn }}$ based upon the radar's bandwidth capabilities, scheduling congestion, and the sensitivity requirements. If the designer is incorporating the CRN technique 


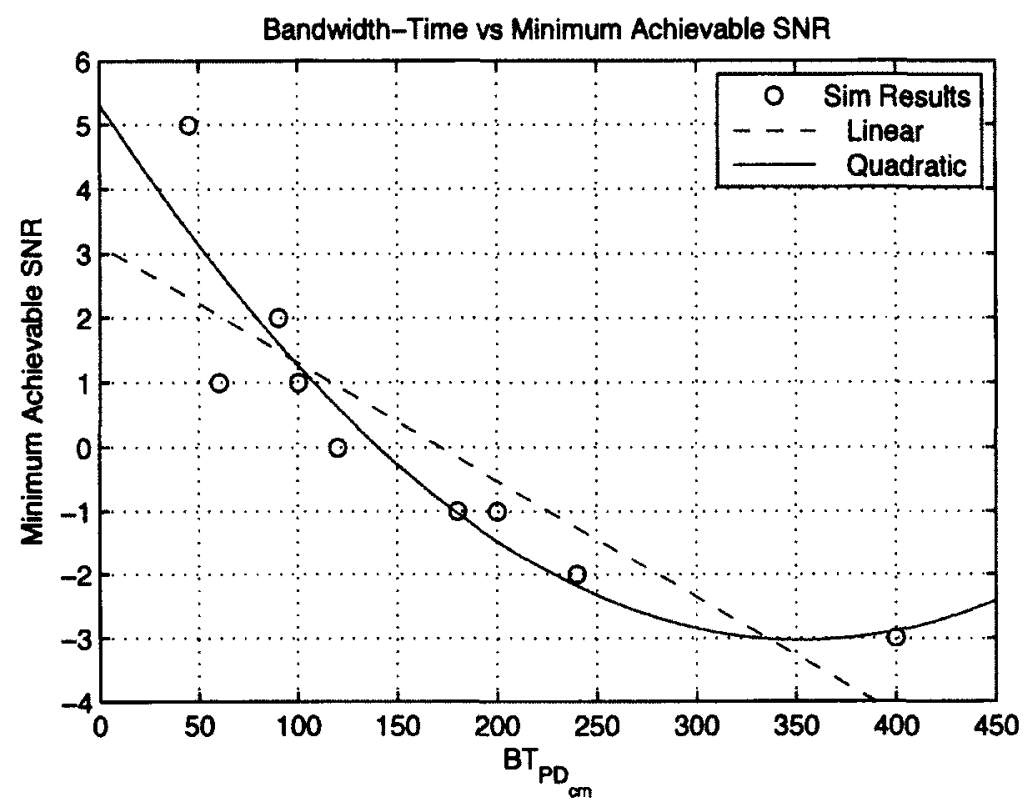

Figure 5.12: Relationship between Bandwidth-Time Product $\left(B T_{P D_{\text {crn }}}\right)$ and Minimum Achievable $S N R_{\text {radarskin }_{\text {sin }}}$

into a tracking radar, where $S N R_{\text {radar }_{\text {skin }}}$ is relatively high, the short pulse can be used and more radar resources can be left for tracking purposes. Conversely, the designer of a search radar may need to detect target with lower $S N R_{\text {radar }_{\text {skin }}}$ values. In this case, the long pulse would be more appropriate.

As a reminder, these results are based on an assumption that $f_{s}=6.667 \mathrm{GHz}$, which was not varied. Some comments should be made about the theoretical effects of changing $f_{s}$. A sampling frequency of this value is pushing the limits of today's most advanced ADCs and DACs, so only lower sampling frequencies will be commented on. The first effect of lowering $f_{s}$ will be to make the pulse duration, $T_{P D_{c r n}}$, longer. The second effect of lowering $f_{s}$ would be on the PDF of the sampling delay, $f_{\tau}(z)$. Essentially, the sampling frequency, or more precisely the sampling period $T_{s}$, is what sets the segment size when constraining $f_{\tau_{q, p}}(z)$ to determine $f_{\tau}(z)$. A lower sampling frequency would cause $f_{\tau}(z)$ to diverge from a uniform distribution. For MPRF, it would remain a uniform distribution as low as $2 \mathrm{GHz}$. For HPRF, however, 
the approximation of a uniform distribution would become increasingly less accurate as $f_{s}$ is lowered. In response, a new PDF would need to replace the uniform distribution currently in the simulation, and the simulation would need to be run again to determine the effectiveness with a lower sampling frequency and the new $f_{\tau}(z)$.

In conclusion, these results prove that the CRN technique is theoretically feasible. Many areas remain to be optimized and some questions are yet to be answered, however, further research into the CRN technique could prove fruitful. 


\section{Chapter 6}

\section{Conclusion}

The aim of this thesis was to develop an effective EP technique that could discriminate between a skin return and DRFM jammer return, without impacting the effectiveness of the radar in its primary role, and by using a vulnerability of the DRFM jammer that the designer could not easily counter. The scope of this thesis was confined to the EP technique, named the CRN technique, and the system by which it would be implemented, named the EP Processor. It was therefore assumed that tasks such as up-converting, down-converting, and isolating the CRN pulse were done by the radar. As well it was assumed that the radar and DRFM had identical DSP capabilities in terms of bandwidth, sampling frequencies, bits and processing speeds.

After a literature review presented in chapter 3 it was determined that research in open literature was lacking in several areas of EP techniques. The main open areas of research are: (1) EP techniques countering cross-eye jamming, (2) EP techniques countering DRFMs that can cope with pulse-to-pulse diversity, and (3) EP techniques that use the contents of a replica pulse, rather than external information such as spatial, temporal, Doppler or average power information.

The research path followed was to exploit the fact that the DRFM jammer must digitize the signals it receives, and that it can only incoherently sample those signals, with respect to the radar transmitter. The latter also applies to the radar receiver, 
however, the fact that this digitization and incoherent sampling will happen twice in the DRFM signal path compared to once in the skin return signal path allows for EP processing.

\subsection{Summary of Contributions}

The principle contributions of this thesis are:

1. A novel EP technique, the Concatenated Random Noise (CRN) technique, which is able to discriminate between DRFM and skin returns.

2. An EP technique that does not require the radar to sacrifice the effectiveness of its primary role by requiring non-optimal waveforms.

3. An EP technique that targets a critical DRFM jammer component that is not easily modified. In other words, an EP technique that is not easily countered without replacement of hardware components, namely ADCs and DACs with $f_{s}>6.667 \mathrm{GHz}$, which may be expensive or not operationally proven yet.

4. An EP technique that could be used to counter DRFM jammer angular deception techniques, such as cross eye.

5. An EP technique that uses the contents of a replica pulse, rather than external information such as spatial, temporal, Doppler or average power information.

6. An EP technique that can counter a DRFM which is able to cope with pulseto-pulse diversity.

7. An EP technique that could aid in reliable identification of jammer signals to ensure that existing jamming signal separation and filtering techniques are only used against jamming signals. 
8. A simulation of the CRN technique that allows for the estimation of its effectiveness with different parameter configurations.

9. A demonstration of the effects of varying the following parameters of the CRN technique: CRN pulse length $N_{c r n}, \mathrm{CRN}$ pulse bandwidth $B, \mathrm{CRN}$ pulse center frequency $\omega_{c}$, and the difference in SNR between the skin return and the DRFM return $\triangle S N R$.

10. A demonstration of reliable identification of skin and DRFM returns, with probabilities of detection $\left(P_{D_{s k i n}}\right.$ and $\left.P_{D_{d r f m}}\right)$ greater than $95 \%$ and probabilities of false alarm $\left(P_{F A_{s k i n}}\right.$ and $\left.P_{F A_{d r f m}}\right)$ less than $5 \%$, in all configurations with $S N R_{\text {radar }_{\text {skin }}}>5 d B$.

11. A demonstration of reliable identification of skin and DRFM returns, at an SNR as low as $S N R_{\text {radar }_{s k i n}}=-3 d B$ with $P_{D_{s k i n}}>98 \%, P_{D_{d r f m}}>98 \%, P_{F A_{s k i n}}<$ $2 \%$, and $P_{F A_{d r f m}}<2 \%$ using a long CRN pulse of 2400 samples and bandwidth of $33 \%$.

\subsection{Future Research}

There are many opportunities for future research and to further explore the feasibility of the CRN technique which are categorized and briefly discussed in the sections below.

\subsubsection{Adaptive Threshold}

A current element missing from the CRN technique is the ability to adapt the threshold of the threshold detector in order to have the highest possible $P_{D_{s k i n}}$ and $P_{D_{d r f m}}$. As can be seen from the results of Chapter 5, the threshold to optimize detection varies with $S N R_{\text {radar }_{s k i n}}$, which is completely out of the EP Processor's control, for 
fixed $B$ and $\omega_{c}$. Radars face a similar problem with their thresholds for target detection, and the common approach to this problem is Constant False Alarm Rate (CFAR) detectors [10]. The CRN technique is slightly more complex due to the fact that it is comparing two signals rather than interference and a signal, where interference includes noise and clutter. This makes it more complex because a regular CFAR detector will always have the interference input to perform continuous CFAR calculations, where the CRN technique does not. However, a suggestion can be made. The radar CFAR detector could share its threshold values with the EP Receiver and the EP Receiver could use that information as an input to an algorithm to determine its own threshold. This would give the EP receiver information on the current interference environment. This would allow for an approximation of $S N R_{\text {radar }_{s k i n}}$ to be made.

\subsubsection{False Targets}

Another topic that was out of scope for this thesis was building a mechanism in the EP Receiver to cope with an interference signal that the radar passes to the EP Receiver. With the current detector configuration, a signal comprised of noise, interference or clutter would be under $T$ and would therefore cause a DRFM false alarm. It is clear that the matched filter response will have a very low maximum magnitude compared to both a skin return and DRFM return since it will not contain $x_{c r n}$ at all.

One could simply add a third hypothesis, $H_{2}$, that means "the measurement, $|y|^{2}$, is a result of interference" and add a guard threshold $T_{g}$ that would be evaluated before the main threshold detector defined in (4.35). This new threshold would be evaluated as follows:

$$
|y|^{2} \underset{H_{2}}{\stackrel{H_{0} \text { or } H_{1}}{>}} T_{g}
$$

The threshold $T_{g}$ would need to be related to $T$ in some way so that it could also 
benefit from a adaptive threshold algorithm as suggested in Section 6.2.1.

\subsubsection{Quantization Noise and Jitter}

The simulation used to generate the results in Chapter 5 does not include any simulation of quantization noise or jitter introduced by DACs and ADCs. It is believed that including this noise and jitter would benefit the EP Processor since there would be more distortion added to $x_{c r n}$ by the extra ADC and DAC in the DRFM signal path. The simulation could be extended to validate this assumption.

\subsubsection{Doppler Shift}

The CRN technique as presented in this thesis does not have a built in mechanism to deal with Doppler shift. In the future, studies could be done to determine the effect of Doppler shift on the CRN technique. This could also include a study of the need for a coping mechanism and as well as a simulation of it. A very brief analysis is offered here.

From [9] we have an approximation of Doppler shift based on radial velocity as follows:

$$
f_{d} \cong \frac{v_{r}}{\lambda}
$$

where $f_{d}$ is the Doppler frequency, $v_{r}$ is the radial velocity of the target in knots and $\lambda$ is the wavelength of the radar signal. To find the value of $\lambda$ we use (6.2), the maximum engagement velocity calculated in Section 4.1.2, $v_{r}=567 \mathrm{~m} / \mathrm{s}=1080 \mathrm{knots}$, and the assumed $f_{s}=6.667 \mathrm{GHz}$. We also assume the radar is using direct digital conversion at the Nyquist rate. This assumption means that the maximum radar signal frequency $f_{r}$ will be $f_{r}=\frac{f_{s}}{2}$ and $\lambda$ is then defined as:

$$
\lambda=\frac{2 c}{f_{s}}
$$


We could then consider the sampling frequency of the received, Doppler shifted signal to be:

$$
f_{s_{\text {Doppler }}}=f_{s}+2 f_{d}
$$

This received signal would be sampled at $f_{s}$ at the radar receiver. The question is whether the sampling frequencies, $f_{s}$ and $f_{s_{\text {Doppler }}}$, are different enough that the EP receiver will sample fewer points than the $\mathrm{EP}$ waveform generator created. If so, the matched filter output magnitude will be significantly lower. The received CRN pulse will be compressed due to the "new" sampling frequency, $f_{s_{\text {Doppler }}}$, and therefore its pulse duration will be slightly less. As long as the difference in pulse durations $\Delta T_{P D}$ is less than $T_{s}$ no samples will be lost. This is shown below, where $N_{c r n}=1200$ :

$$
\begin{aligned}
T_{P D} & =N_{c r n} \cdot T_{s} \\
& =N_{c r n} \cdot \frac{1}{f_{s}} \\
T_{P D_{\text {Doppler }}} & =N_{c r n} \cdot T_{s_{\text {Doppler }}} \\
& =N_{c r n} \cdot \frac{1}{f_{s_{\text {Doppler }}}} \\
\Delta T_{P D} & =T_{P D}-T_{P D_{\text {Doppler }}}
\end{aligned}
$$

Combining (6.2), (6.3) (6.4), (6.5), (6.6), and (6.7) results in:

$$
\begin{aligned}
\Delta T_{P D} & =\frac{N_{c r n}}{f_{s}}\left(1-\left(1+\frac{v_{r}}{c}\right)^{-1}\right) \\
& =648 f s
\end{aligned}
$$

Since $\Delta T_{P D} \ll T_{s}=150 p s$, no samples will be lost, and theoretically the CRN technique could easily cope with Doppler shift. However, this should be confirmed through simulation using different values of $N_{c r n}$ and $f_{s}$.

If it is found that the CRN technique does require a coping mechanism for Doppler 
shift, there are mechanisms commonly used by radars described in [10]. These could possibly be implemented in the EP Processor and then simulated to determine their merit.

\subsubsection{Lower Bandwidth Requirements}

As mentioned in Section 5.1.4, a problem was encountered where noise was being introduced by the demodulator when the bandwidth was set to $B=0.1$. After some troubleshooting it was determined that this was likely due to the fact that the CRN simulation was not windowing the CRN pulse to allow for operation at lower bandwidths. Therefore, an area for future research would be to add a windowing function to the CRN technique. This would be most appropriately added to the decatenator module. The radar receiver would already be doing some processing to segregate the CRN pulse from the radar pulse. It is believed that this would reduce the amount of noise power entering the matched filter and would, therefore increase the sensitivity of the matched filter and allow for operation at bandwidths lower than $B=0.15$.

\subsubsection{Demonstrate for Different Radar Types}

Demonstrating that the CRN technique works with different radar types would add to its acceptance as a feasible EP technique. The CRN technique presented in this thesis is simulated on a very generic radar. This radar is assumed to use Coherent Processing Intervals (CPIs) which the EP Processor conforms to by integrating data across the same CPIs. This CPI technique is usually used in search radars. Since the CRN technique does not require coherent processing it could be ported to other radar types. 
As well, there were elements omitted from the EP Processor design and development of the $f_{\tau}(z)$, such as pulse staggering which is commonly done in search radars. This would add another dynamic to $f_{\tau}(z)$ and also to the CRN technique that may be to the EP processors advantage. Only further research could reveal this.

A significant amount of work is required in customizing the CRN technique and EP processor to match the host radar. This author believes that they are sufficiently flexible to allow for this and encourages future research in customizing the CRN technique and EP Processor to work with representative radars of many functions, frequency bands and design.

It would be interesting to model a tracking radar using the CRN technique against a DRFM using the cross-eye technique. The platform performing cross-eye jamming is actually recording each CRN pulse twice and retransmitting each, just out of phase. This gives the radar two opportunities to improve the EP of the CRN technique. First, the radar is receiving twice as many CRN pulses for processing, assuming they can be gleaned from each antenna before they are mixed into sum and difference channels. Second, the phase distortion added by the jammer may add more distortion to the CRN pulse which could allow for higher detection probabilities or faster detection times. These would need to be simulated to confirm these added EP opportunities.

\subsubsection{Optimization of Integration Scheme}

For this thesis, an integration scheme was chosen that provided favourable results. However, that integration scheme was never optimized to ensure the most effective discrimination between skin returns and DRFM returns. This makes it possible for higher probabilities of detection to be achieved, for reliable detection to be done at lower SNRs, and for detection decisions to be made faster. In other words, with fewer CRN pulses. Analysis and simulation of the integration scheme could reveal efficiencies to be made. 


\subsubsection{Simulation in Hardware with an Analog Signal Path}

Once the above research has been completed with favourable results, the CRN technique may be ready for hardware implementation and more real-world simulation. Implementing the technique in a DSP or FPGA, and converting the signal from the digital to analog domains and back would be required to begin to prove the operational feasibility of the CRN technique.

\subsubsection{Determine $f_{\tau}(z)$ for Land, Naval and Space Engage- ments}

This thesis focused on fighter aircraft employment of the CRN technique when developing $f_{\tau}(z)$. This was done so that the PDF would be a uniform distribution. However, it is quite likely that the CRN technique will work with other types of PDFs. It is thought that the sampling delay, $\tau$, for less dynamic engagements such as land, naval and space engagements will vary much less pulse-to-pulse. This means that if the first pulse of a CPI had a delay of $\tau=\frac{T_{s}}{2}$ it may slowly progress to a delay of $\tau=\frac{T_{s}}{3}$ by the final pulse of the CPI. Conversely, the first pulse could start at $\tau=0$ and progress to a delay of $\tau=\frac{T_{s}}{3}$. The first case would be beneficial to the CRN technique but the second case would be detrimental. An opportunity for future research would be to develop an appropriate $f_{\tau}(z)$ for these situations, incorporate it into the CRN simulation and see if the results are favourable.

\subsubsection{Integration with Existing EP Techniques}

If the CRN technique were to be proven operationally feasible in hardware, then research could be undertaken to integrate this technique with existing techniques outlined in Chapter 3. The integration of multiple EP techniques within a radar 
could add to the overall effectiveness of the radar's EP suite.

\subsubsection{Advanced Tracking}

With the CRN technique, you now know which return is the target and which return is from the jammer mounted to that target. Using this information the target can be tracked with greater accuracy than without the jammer signal present. Kalata and Chmielewski provide an $\alpha-\beta$ tracker for this purpose in [23]. Combining this tracker with the CRN technique could allow for more accurate tracking, and perhaps for a new missile guidance mode, where small corrections are sent to the missile based on the tracking information from this $\alpha-\beta$ tracker.

\subsubsection{Radar Resource Allocation}

While this technique requires the use of precious radar resources, advances in other areas may make this an acceptable trade-off. There are three areas where future research could be applied to help open up scheduling time required by this technique.

The first would require the more efficient use of radar resources across many radars. The next generation fighter aircraft will benefit from highly capable data links and data fusion engines in their mission computers. This could give a group of platforms the ability to coherently schedule a group of radars to share the workload and share the results through their data links. This will free up resources for EP processing to increase the reliability of all radars.

The second would require the same data link capabilities between platforms, but would reduce radar resources, or simply improve detection probabilities, by adding another level of integration-at the platfrom level. Conceptually, the data links would allow radars to share EP receiver results across multiple platforms. The platforms 
could perform non-coherent or binary integration on the cross platfrom data to improve performance metrics, or allow for detection of DRFMs at lower SNRs, and therefore longer ranges. Using integration across platforms allows for greater spatial diversity, which would statistically be in the EP receivers' advantage.

The third would make scrupulous use of the CRN technique. Theoretically, the technique could be operator controlled or cued by other algorithms in order to make the most efficient use of radar resources. For example it could be combined with the technique in Section 3.1.4, which uses frequency diversity to discriminate between Rayleigh and fixed amplitude echoes, and therefore between target and DRFM jammer returns. The radar could use the frequency diversity technique all the time and set it to be fairly sensitive. When a possible jammer is detected, the CRN technique could be included to positively determine if jamming is present. This would help that radar to more efficiently schedule its time. 


\section{List of References}

[1] R. E. Fitts, editor. The Strategy of Electromagnetic Conflict. Peninsula Publishing (1980).

[2] D. C. Schleher. Electronic Warfare in the Information Age. Artech House (1999).

[3] D. Adamy, J. Harding, G. Carlson, N. Balchunas, and V. Battaglia. "Essentials of Electronic Warfare." (2009).

[4] B. Manz. "DRFMs grow to meet new threats." Journal of Electronic Defense 33(8), 43 (2010).

[5] D. DiFilippo, G. Geling, and G. Currie. "Simulator for advanced figher radar epm development." In "IEE Procedings - Radar, Sonar, and Navigation," volume 148 , pages 139-146 (2001).

[6] F. Neri. "Anti-monopulse jamming techniques." In "Proceedings of the 2001 SBMO/IEEE MTT-S International Microwave and Optoelectronics Conference," IEEE (2001).

[7] L. Falk. "Cross-eye jamming of monopulse radars." In "Waveform Diversity and Design Conference," IEEE (2007).

[8] J. G. Proakis. Digital Signal Processing: Principles, Algorithms and Applications. Pearson Education (2007).

[9] M. L. Skolnik. Introduction to Radar Systems. McGraw Hill (2001).

[10] M. A. Richards, J. A. Scheer, and W. A. Holm. Principles of Modern Radar: Basic Principles. Scitech Publishing (2010).

[11] A. Leon-Garcia. Probability, Statistics, and Random Processes for Electrical Engineering, chapter 7, page 361. Pearson Prentice Hall, third edition (2008). 
[12] G. Lu, D. Zeng, and B. Tang. "Anti-jamming filtering for DRFM repeat jammer based on stretch processing." In "2nd Internation Conference on Signal Processing Systems," IEEE (2010).

[13] M. Schikorr. "High range resolution with digital stretch processing." In "IEEE Radar Conference," IEEE (2008).

[14] D. Wehner. High Resolution Radar. Artech House, second edition edition (1995).

[15] G. W. Stimson. Introduction to Airborne Radar, chapter 35, pages 461-462. SciTech Publishing (1998).

[16] D. D. Howard. "High range-resolution monopulse tracking radar." Technical report, Naval Research Laboratory (1975).

[17] X. Fu, C. Jiang, Z. Wang, and M. Gao. "Anti-vessel end-guidance radar ECCM against deception jamming of range gate pull off." In "International Radar Conference," IEEE (2009).

[18] W. Blair and M. Brandt-Pearce. "Discrimination of targets and RGPO echoes using frequency diversity." In "Proceedings of the Twenty-Ninth Southeastern Symposium on System Theory," IEEE (1997).

[19] J. Akhtar. "An ECCM scheme for orthogonal independent range-focusing of real and false targets." In "IEEE Radar Conference," IEEE (2007).

[20] "Sorbtsiya radar jammer." Jane's Avionics (2010).

[21] D. S. Garmatyuk and R. M. Narayanan. "ECCM capabilities of an ultrawideband bandlimited random noise imaging radar." IEEE Transactions on Aerospace and Electronic Systems 38(4), 1243-1255 (2002).

[22] R. M. Narayanan. "Random noise monopulse radar system for covert tracking of targets." Technical report, Nebraska University Lincoln Department of Electrical Engineering (2002).

[23] P. R. Kalata and T. A. Chmielewski. "Range gate pull off (rgpo): Detection, observability and alpha - beta target tracking." In "Proceedings of the TwentyNinth Southeastern Symposium on Systems Theory," IEEE (1997). 


\section{Appendix A}

\section{Random Delay for HPRF Radars}

Here we will show the development of $f_{\tau}(z)$ for the HPRF case, following the same process as Section 4.1.2.

The development will begin with the same definition of $f_{v}(y)$ from (4.4). Figure A.1(a) shows $f_{v}(y)$, while figure A.1(b) shows the amount the range can change due to the velocity during one PRI, $f_{\triangle R_{P R I}}(y)$. The transformation from (a) to (b) is from (4.5) where $T_{P R I}=10 \mu \mathrm{s}$. In essence, this is a change in units from velocity to range. Therefore, the transformation is applied to both $\mu$ and $\sigma$. The result is $\mu_{\Delta R_{P R I}}=-1.53 \mathrm{~mm}, \sigma_{\Delta R_{P R I}}=1.5 \mathrm{~mm}$, and $f_{\triangle R_{P R I}}(y)$ is given in (4.6). Finally, figure A.1(c) shows $f_{V_{q}}(y)$, the delay that will be caused, negative or positive, during one PRI. The transformation from (b) to (c) is given in (4.7). Again, this is basically a change in units, from range to time and therefore $\mu_{V_{q}}=-5.1 p s, \sigma_{V_{q}}=5.0 p s$, and $f_{V_{q}}(y)$ is defined by (4.8). Therefore, from the relative velocities of the radar and target we have defined $V_{q}$, the random variable that contributes to delay based on velocity.

Second, let us analyse the $R_{q}$ term. To determine the $f_{R_{q}}(x)$, we need to look at $\Delta R_{C P I}$, the change in range between CPI $q$ and CPI $q+1$. The same methodology for the derivation of $f_{V_{q}}(y)$ will be used. Figure A.2(a) shows the $f_{v}(y)$ again, from (4.4), figure A.2(b) shows $f_{\Delta R_{C P I}}(x)$ which shows the amount the range can change 

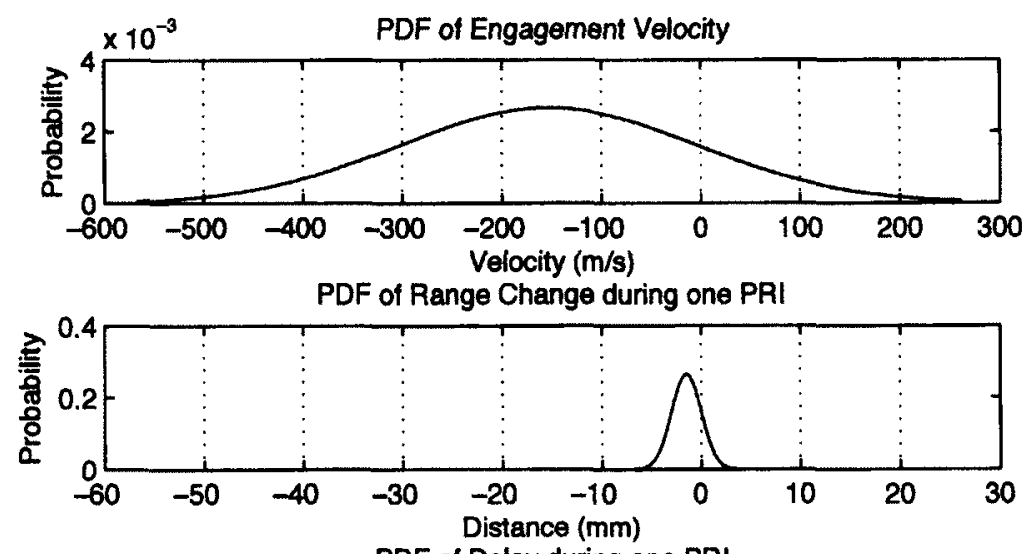

PDF of Delay during one PRI

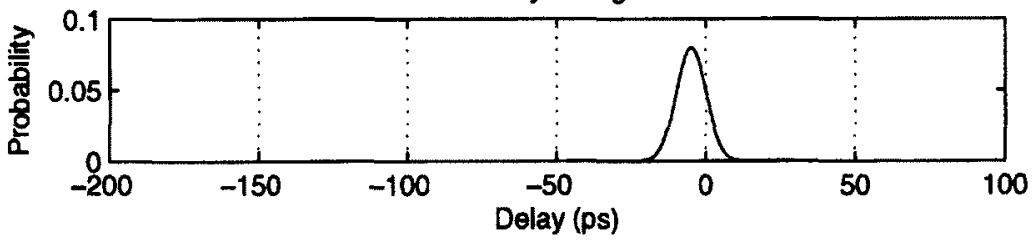

Figure A.1: Development of $f_{V_{q}}(y)$, HPRF Case. (a) PDF of Engagement Velocity, $f_{v}(y),(\mathrm{b})$ PDF of Range Change during one PRI $f_{\Delta R_{P R I}}(y),(\mathrm{c}) f_{V_{q}}(y)$

over the course of a CPI. It is derived from (a) with the following equation (4.9) where $N_{C P I}=10$ is the number of PRIs in a CPI. For the purposes of this thesis, it is assumed that $N_{C P I}=10$. Therefore, the result is $\mu_{\triangle R_{C P I}}=-15.3 \mathrm{~mm}, \sigma_{\triangle R_{C P I}}=$ $15 \mathrm{~mm}$, and $f_{\triangle R_{C P I}}(x)$ is defined by (4.10). The overall delay that can occur during a CPI is shown in figure A.2(c) and was derived from (b) with the following equation (4.11). The result is $\mu_{R_{q}}=-51 p s, \sigma_{R_{q}}=50 p s$, and $f_{R_{q}}(x)$ is given by (4.12).

From the relative velocities of the radar and target we have defined $R_{q}$, the random variable that establishes the amount of delay possible, from CPI $q$ to CPI $q+1 . R_{q}$ is meant to establish the initial delay value for a CPI, however, as can be seen in the PDF, the value of $R_{q}$ could easily exceed the maximum delay value established in (4.1), $T_{s}$. This will be addressed next after we resolve addition of the 2 PDFs.

Equation (4.13) gives the addition of the PDFs and defines $f_{\tau_{q, 1}}(z)$. Figure A.3 shows (4.13) applied. 


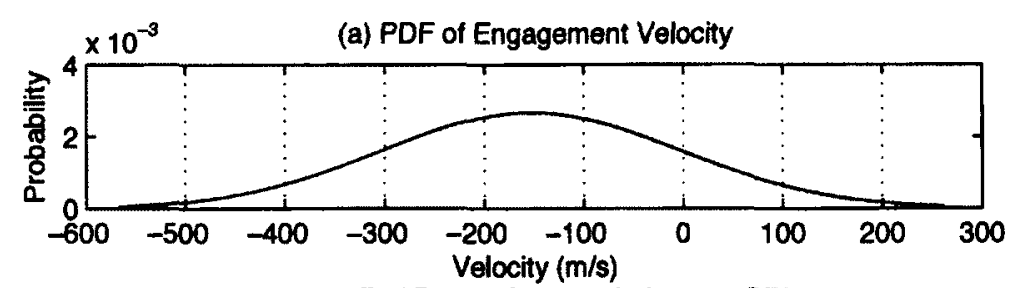

(b) PDF of Range Change during one CPI

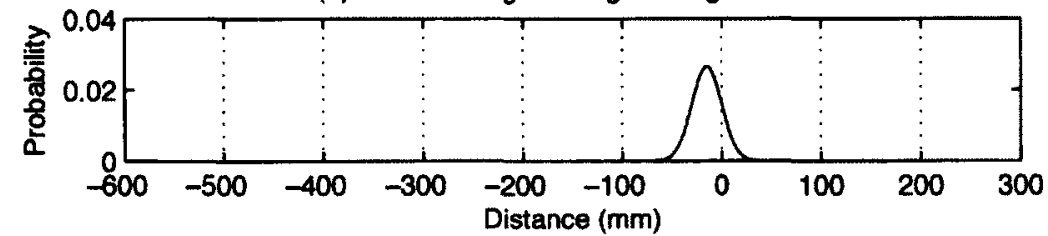

(c) PDF of Delay during one CPI

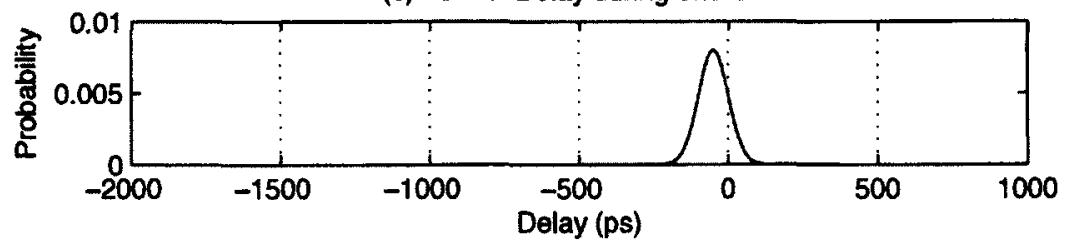

Figure A.2: Development of $f_{R_{q}}(x)$, HPRF Case. (a) PDF of Engagement Velocity, $f_{v}(x)$ (b) PDF of Range Change during one CPI $f_{\Delta R_{C P I}}(x),(\mathrm{c}) f_{R_{q}}(x)$

However, the overall delay is not very important. What is more important is where the delay will fall within one sample period. Figure A.4(a) shows $f_{\tau_{q, 1}}$ and divides it into segments $T_{s}$ wide. The additive probabilities of all the segments are shown in (b) as well as the ideal uniformly distributed PDF. The result is that $\tau_{q, 1}$ is approaching a uniformly distributed random variable with a probability of approximately $\frac{1}{T_{s}}=$ $\frac{1}{150}=6.667 \times 10^{-3}$.

However, it needs to be proven that the more general $\tau_{q, p}$ can be simulated by a uniformly distributed random variable for all values of $q$ and $p$. Figures A.5 and A.6, show $f_{\tau_{q, 5}}(z)$ and $f_{\tau_{q}, 9}(z)$ respectively. Both are also approaching a uniformly distributed PDF with probability of approximately $\frac{1}{T_{s}}$ as was $f_{\tau_{q, 1}}(z)$. In fact, as $p$ increases the approximation gets slightly more accurate.

It has therefore been proven that, for the HPRF case with $T_{P R I}=10 \mu s$, $N_{C P I}=10$ and $f_{s}=6.667 G H z, \tau_{q, p}$ can be approximated and modelled as a uniformly 
(a) PDF of Delay during one CPI due to Range

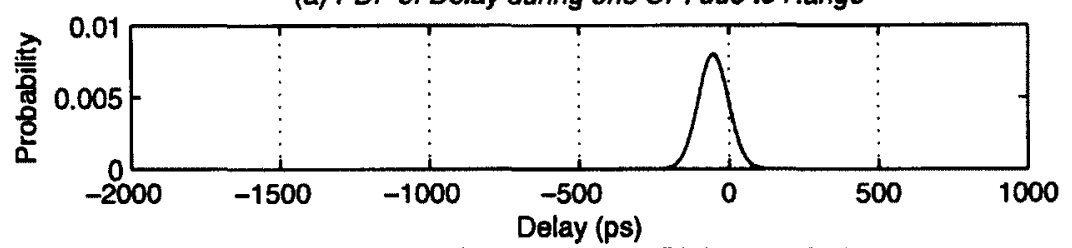

(b) PDF of Delay during one PRI due to velocity

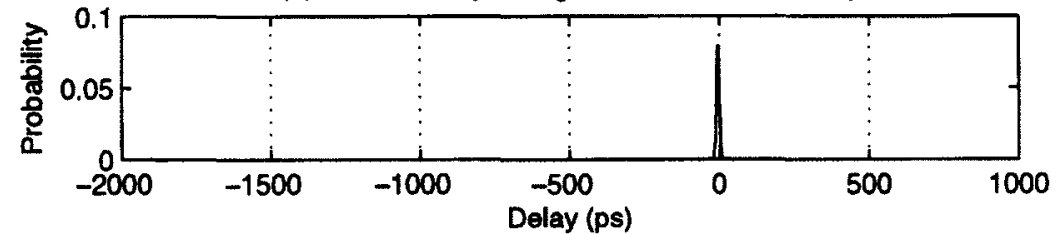

(c) PDF of tau for $p=1$

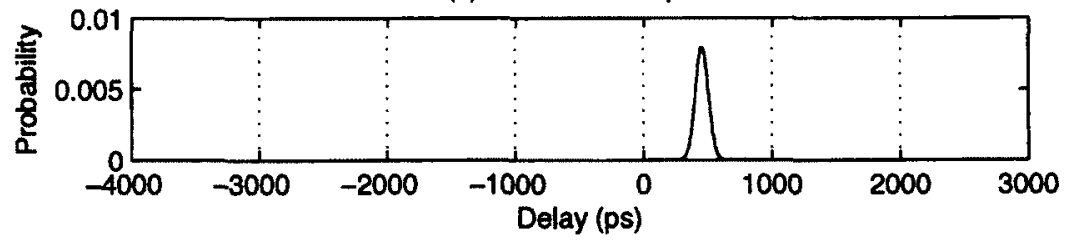

Figure A.3: Development of $f_{\tau_{q, 1}}(z)$, HPRF Case (Unconstrained). (a) $f_{R_{q}}(x)$, (b) $f_{V_{q}}(y),(\mathrm{c}) f_{\tau_{q, 1}}(z)$ (Unconstrained)

distributed random variable with a probability of $\frac{1}{T_{0}}$. However, this approximation will become less realistic if $f_{s}$ or $T P R I$ are decreased. Therefore, this approximation must be used carefully. 

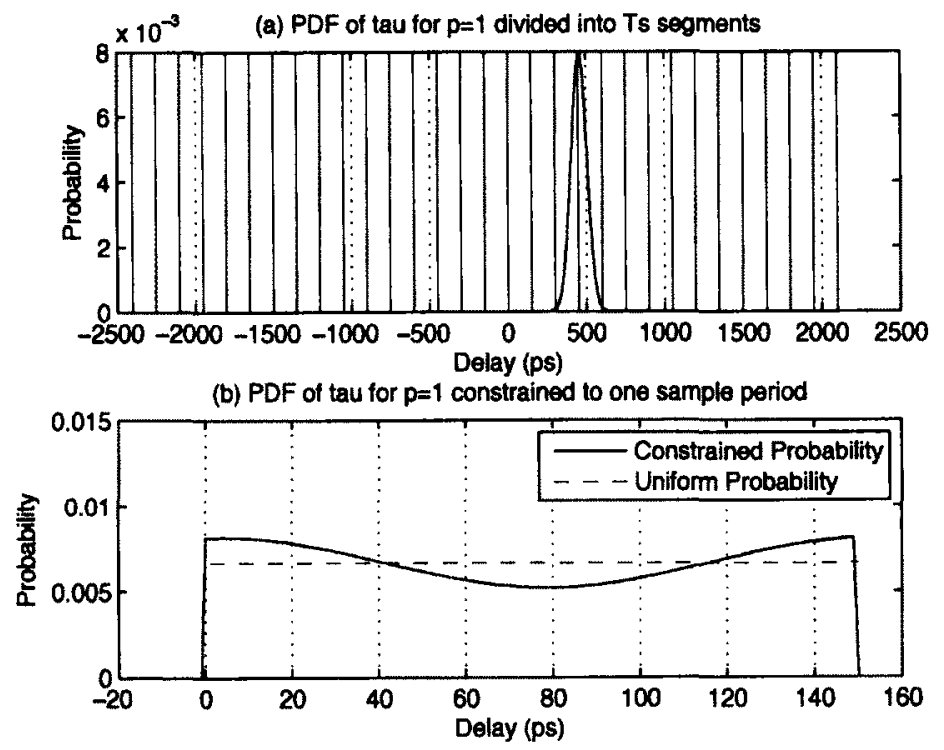

Figure A.4: Development of $f_{\tau_{q, 1}}(z)$, HPRF Case (Constrained to one sample period). (a) $f_{\tau_{q, 1}}(z)$, divided into $T_{s}$ segments, (b) $f_{\tau_{q, 1}}(z)$ (Constrained to one sample period, $T_{s}$ )
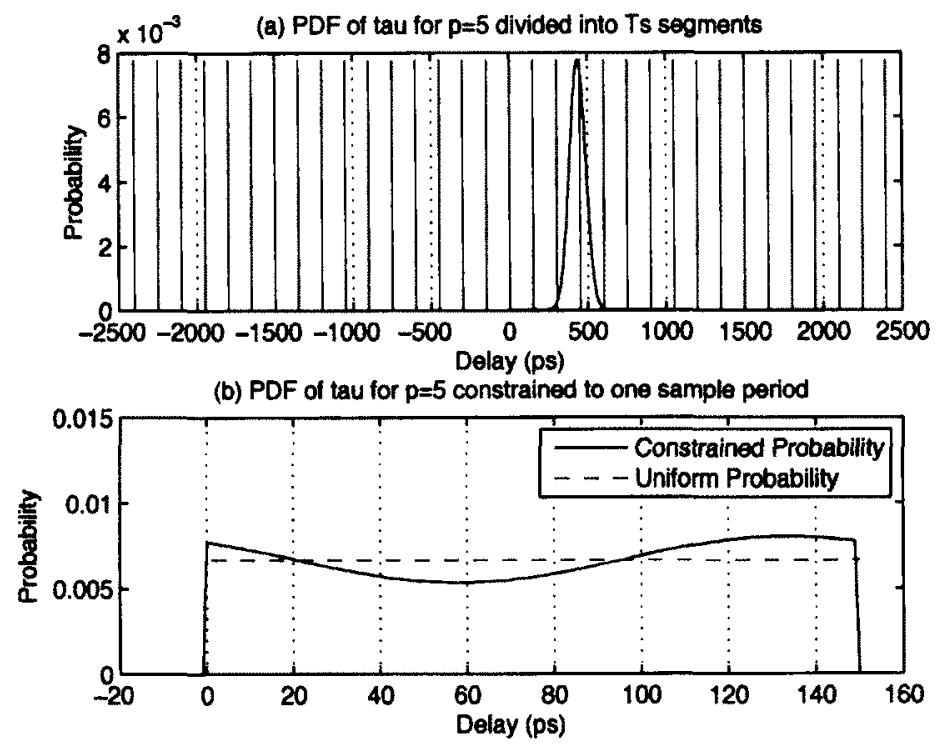

Figure A.5: Development of $f_{\tau_{q, 5}}(z)$, HPRF Case (Constrained to one sample period. (a) $f_{\tau_{q, 5}}(z)$, divided into $T_{s}$ segments, (b) $f_{\tau_{q, 5}}(z)$ (Constrained to one sample period, $T_{s}$ ) 

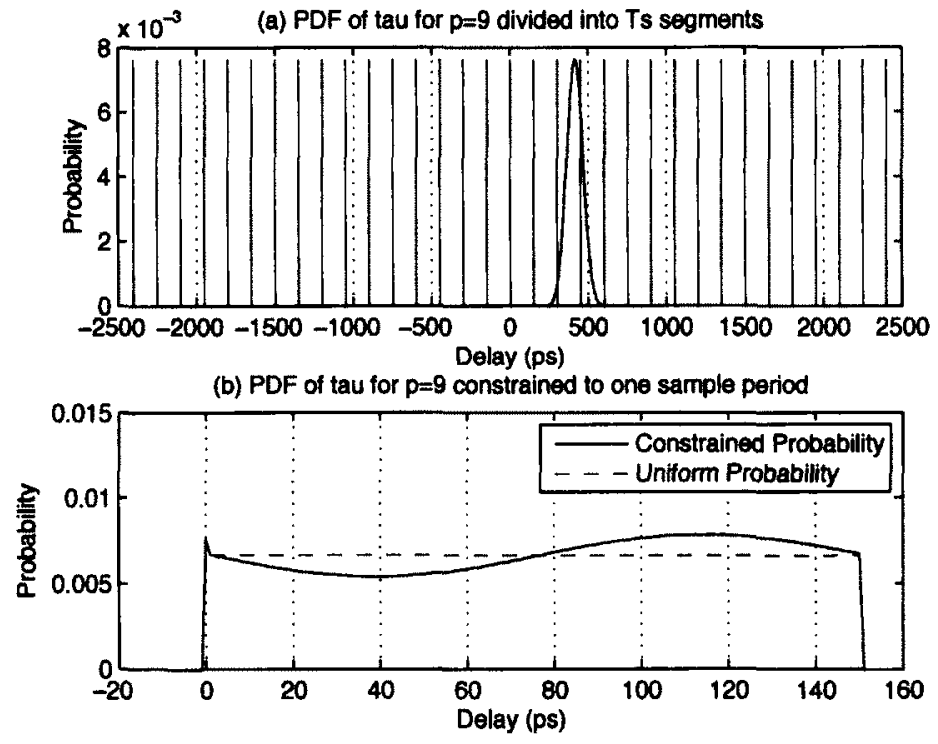

Figure A.6: Development of $f_{\tau_{q, 9}}(z)$, HPRF Case (Constrained to one sample period). (a) $f_{\tau_{q, 9}}(z)$, divided into $T_{s}$ segments, (b) $f_{\tau_{q, 9}}(z)$ (Constrained to one sample period, $T_{s}$ ) 